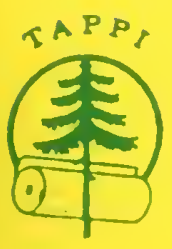

\author{
TECHNICAL ASSOCIATION OF THE \\ PULP AND PAPER INDUSTRY
}

COLLABORATIVE REFERENCE PROGRAM FOR PAPER

REPORT N0.57S

STRENGTH TESTS

U.S. DEPARTMENT OF COMMERCE National Bureau of Standards 


\section{TAPPI Paper and Board (6 times per year)}

Bursting strength

Tearing strength

Tensile breaking strength

Elongation to break

Tensile energy absorption

Folding endurance

Stiffness

Air resistance

Grammage

\author{
Smoothness \\ Surface pick strength \\ $\mathrm{K}$ \& $\mathrm{N}$ ink absorption \\ $\mathrm{pH}$ \\ Opacity \\ Blue reflectance (brightness) \\ Specular gloss, $75^{\circ}$ \\ Thickness \\ Concora (flat crush) \\ Ring crush
}

FKBG-API Containerboard (48 times per year)

Mullen burst of linerboard

Concora test of medium

MCCA Color and Appearance (4 times per year)

Gloss at $60^{\circ}$

Color and color difference

CTS Rubber ( 4 times per year)

Tensile strength, ultimate elongation and tensile stress Hardness

Mooney viscosity

Vulcanization properties

CTS Thermal Insulation Materials (2 times per year)

19 test methods for thermal insulation materials covering:

thermal properties; strength properties; dimensions, stability, and density properties; fire properties; and properties of vapor barriers

ASTM Cement (2 times per year)

Chemical (11 chemical components)

Physical ( 8 characteristics)

\section{AASHTO Bituminous}

Asphalt cement ( 2 times per year)

Cutbacks (once a year)

NBS Collaborative Reference Programs

A05 Technology Building

National Bureau of Standards

Washington, DC 20234 
TECHNICAL ASSOCIATION OF THE

PULP AND PAPER INDUSTRY

\title{
COLLABORATIVE REFERENCE PROGRAM FOR PAPER
}

\author{
Report No. $57 \mathrm{~S}$ \\ STRENGTH TESTS
}

R. G. Powell

TAPPI-NBS Research Associate

Collaborative Testing Services, Inc.

J. Horlick

Office of Testing Laboratory Evaluation Technology

Office of Engineering Standards

National Engineering Laboratory

NBSIR $79-1370$

U. S. DEPARTMENT OF COMMERCE

National Bureau of Standards 
INTREDUCTION

Reports 57.5 and $57 G$ comprise the third set of reports for the 78-79 program yearo Participants in tests which involve strength properties of paper only the S report; those in tests which measure other properties will recelve only the G report.

Notes and comments to individual laboratories and "Best Values" applicable to a particular nethod are given following Table 1 for each method, See page 4 of this report for an explanation of "Best Values". Please do not confuse these Best Values with provisional values included with the samples to detect serious discrepancies at the time of test.

If there are any questions on the notes, the analyses, or the reports in general, contact Robert G. Powell or Jeffrey Horlick on 301/921-2946。

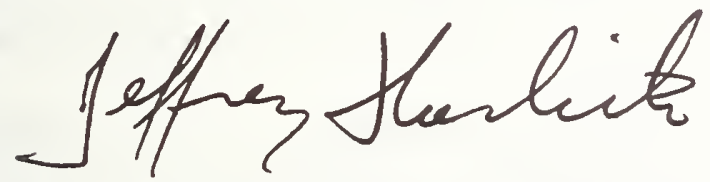

Jeffrey Horlick, Administrator NBS-TAPPI CoLlaborative Reference Program office of Testing Laboratory Evaluation Technology

Apri2 23, 1979 
In 1969, the National Bureau of Standards and the Technical Assocfation of the Pulp and Paper Industry established a collaborative reference program to provide a participating laboratory with a means to check perlodically the level and uniformity of its testing in comparison with that of other laboratories.

The interchange of paper and board products and of the raw materials for these products requires agreement among raw material suppliers, paper and board producers, converters, distributors, retallers, commerclal testing laboratorles, user organizations and the ultimate consumer as to the meaning of test results, an agreement that cannot be achieved without accurate and precise testing. This program is designed to help assure agreement.

\section{HOW THE PROGRAM WORKS}

Participants Select the Tests in which they wish to participate. This choice is made on jolning the program, but additional tests may be added at any time. Also new participants may enter the program at any time.

Test Samples are Distributed Bimonthly; 1.e. every 2 months.

Provisional Values are Provided with the Samples for one or both of the test levels, dependIng on method. The provisional values permit serlous discrepancles to be detected without delay. (It 18 left to the discretion of the laboratory supervisor as to whether these values should be known to the operator.)

Each Participant Tests the Samples, following instructions provided for each test method. The full check on a single instrument should normally take no more than 30 minutes. The test results are then sent to NBS for analysis. The participant is also asked to report other information relevant to an accurate analysis, such as test conditions and the instruments used.

Industry Means, Best Values and Other Stat1stics are developed from the data by NBS. The best values are estimates based on a careful examination of all data, both current and past, with special attention to results obtalned by the National Bureau of Standards and other recognized reference laboratories in this and other countries.

A Quick Report is Prepared for each particlpating laboratory reporting data on time. This report shows the Industry mean values, and the deviations of the laboratory's results from these values for each test method.

A Longer Sumary Report, Showing the Data from all Participants, is also prepared. In the summary report, of which this report is an example, each laboratory is identifled by a code number so that the information is maintained on a confidential basis. However, instruments are Identifled by type so participants can compare their results with those obtained on simflar instruments of different manufacture. This report includes test averages, best values and standard deviations for Individual particlpants and for the group as a whole. A particlpant should be able to readily determine the level and varlability of his results in comparison with those of the other laboratories.

Repeatability and Reproducibility Statements such as Contained in ASTM, TAPPI and Iso Standards are included at the end of the report. Participants can check their performance level against the precision statement given in the test method or specification. 
TABLE OF CONVERSION FACTORS TO METRIC (SI) UNITS

Physical

Quantity

Bursting strength

Tearing strength

Tensile strength

Tensile energy absorption

Bending stiffness

Flat-crush strength (Concora)

Ring-crush (TAPPI)

(ISO)

Thickness
To Convert

From

psi

$\mathrm{kg} / \mathrm{cm}^{2}$

bar

g

lb/in.

1b/0.5 in.

$1 \mathrm{~b} / 15 \mathrm{~mm}$

$\mathrm{kg} / 15 \mathrm{~mm}$

$\mathrm{kg} / 25 \mathrm{~mm}$

$\mathrm{kg} / \mathrm{mm}$

$f t-1 b / f t^{2}$

in. $-1 \mathrm{~b} / \mathrm{in}$. $^{2}$

$\mathrm{kg}-\mathrm{m} / \mathrm{m}^{2}$

$\mathrm{g} \cdot \mathrm{cm}$

$1 b$

$1 b$

$1 \mathrm{~b} / 6.00 \mathrm{in}$.

$\operatorname{mil}$
To

$\mathrm{kPa}$

$\mathrm{kPa}$

$\mathrm{kPa}$

$\mathrm{mN}$

$\mathrm{kN} / \mathrm{m}$

$\mathrm{kN} / \mathrm{m}$

$\mathrm{kN} / \mathrm{m}$

$\mathrm{kN} / \mathrm{m}$

$\mathrm{kN} / \mathrm{m}$

$\mathrm{kN} / \mathrm{m}$

9.807

$\mathrm{J} / \mathrm{m}^{2}$

14.59

$\mathrm{J} / \mathrm{m}^{2}$

175.1

$\mathrm{J} / \mathrm{m}^{2}$

9.807

$\mu \mathrm{N} \cdot \mathrm{m}$

98.07

N

N

$\mathrm{kN} / \mathrm{m}$

$\mu \mathrm{m}$

25.40 
MEAN -

GRAND MEAN -

(GR. MEAN)

SD OF MEANS -

(SD MEANS)

$\mathrm{DEV}-$

N. DEV -

SDR -

AVERAGE SDR -

R. SDR -
The average of individual TEST DETERMINATIONS. The number of TEST DETERMINATIONS in the mean is given in the upper right corner of the first table (TEST D.) and again at the bottom of this table.

The average of the individual laboratory MEANS, excluding laboratories flagged (see column $F$ ) with an $X$, $\#$, or + . The GRAND MEAN is given in US customary units and, where applicable, in SI metric units.

The standard deviation of the laboratory MEANS about the GRAND MEAN; an index of the among-laboratory precision.

The deviation or difference of the laboratory MEAN from the GRAND MEAN.

The normal deviate or ratio of the DEV to the SD OF MEANS; an indication of the degree of divergence of the laboratory MEAN from the GRAND MEAN. A N. DEV of more than 2 or less than -2 may indicate that the participant is not following the procedure considered standard for this analysis.

The standard deviation of repeated measurements; that is, of individual test determinations about their MEAN.

The average of the individual laboratory SDR's; an index of the-within-laboratory precision of repeated measurements.

The relative standard deviation of repeated measurements; that is, the ratio of the SDR to the AVERAGE SDR: an indication of the ability of a participant to repeat his measurements relative to the average ability. The greater the number of TEST DETERMINATIONS the closer the R. SDR should be to unity. If R. SDR is outside the limits given below, the participant may not be following the procedure considered standard for this analysis: 
No. of test

Determinations

3

5

8

10

15

20

25
Lower linit

for R. SDR

0.09

0.27

0.40

0.46

0.56

0.61

0.65
Upper limit

for R. SDR

2.58

2.06

1.77

1.67

1.53

1.45

1.39
VAR -

F -

$+$

\#-

M -

X -

S -

0 -

COORDINATES -
Code for instrument type or variation in condition, see second table.

Flag, with following meaning:

Excluded from grand means because VAR non-standard for this analysis

Excluded because data were not understood or because of a non-coded variation reported by the laboratory. (See NOTES following Table 1 for each method.)

Excluded because data for one sample are missing

Excluded because plotted point would fall outside of the $99 \%$ error ellipse, (see below for explanation of Graph)

Included in grand means but plotted point falls outside of the $95 \%$ error ellipse.

The participant should take this as a warning to reexamine his testing procedure

Included in grand mean but only after omission of one of more 'wild' values;

that is, test determinations more than 3

times AVERAGE SDR from the laboratory's

MEAN. Not more than 20\% of the test determination may be excluded in this manner without rejecting the laboratory.

Included in grand mean and inside $95 \%$ error ellipse.

Distances along major and minor axes of error ellipse. If special additive or concurrent model of the measuring process applies to this method, the distance along the minor axis represents the random error within a laboratory while that along the major axis also includes a systematic laboratory component of error. 
95\% ELLIPSE -

AVG R. SDR -

Graph -
Lengths of the major and minor axes of the ellipse and the angle that the major axis makes with the horizontal axis.

Average of the R. SDR for the two samples; an indication of the laboratory's precision of repeated measurements.

For each laboratory the MEAN for the second sample is plotted against the MEAN for the first sample, with each point representing a laboratory. The horizontal and vertical lines are the GRAND MEANS. The dashed line is drawn at $45^{\circ}$. The solid sloping line, which may or may not lie close to the $45^{\circ}$ line, is along the major axis of the error ellipse. The ellipse is drawn so that, on the average, it will include $95 \%$ of the points representing the laboratories.

Plotted symbols are as explained above (under F), except that an ' $S$ ' is plotted as an ' $O$ '. A participant whose plotted point falls outside of the ellipse should carefully reexamine the testing procedure he is following.

The graph is plotted with an ellipse when there are 20 or more laboratories in the analysis. When there are 10 through 19 laboratories in the analysis the graph is plotted but the ellipse is omitted. When there are fewer than 10 laboratories retained in the analysis the graph is not plotted.

The International System of Units (SI) is used on the plots wherever possible to aid participants in familiarizing themselves with SI. Grand means in SI units are given at the top of the plot, and supplementary scales in SI units are drawn along the axes allowing the reader to compare means and variability in common units and SI units for the same data. 
Summary -
(At end of
report)

REPL CRP -

REPL TAPPI -

REPEAT -

REPROD -

Best values -
In addition to several quantities already defined above the summary shows the following values for each test method:

The number of replicate test determinations used in this Collaborative Reference Program.

The number of replicate test determinations in a test result required by the applicable TAPPI Standard or assumed here if there is no TAPPI Standard. This quantity is needed in the computation of TAPPI repeatability and reproducibility from the SD OF MEANS and the AVER SDR. See TAPPI Standard T1206 for definitions and computations.

TAPPI repeatability, a measure of the withinlaboratory precision of a test result.

TAPPI reproducibility, a measure of the betweenlaboratory precision of a test result.

Given at the end of Table 1 for each method for which sufficient information is available. These best values are estimates based on a careful examination of all data, both current and past, with special attention to results obtained by the National Bureau of Standards and other recognized reference laboratories in this and other countries. All participants using equipment that is standard for the analysis should be able to achieve results within the plus-minus ( \pm ) limits, when these are shown along with the best values. 
NALIBIS I10-1 TABLE

IAPPI SIANDARD T 403 68-76. BURETINO BTRBNGTE OF PAPBR - PBREINB MEDEL C

\begin{tabular}{|c|c|c|c|c|c|c|c|c|c|c|c|c|c|}
\hline & SAMPLB & & PRINI & & & BAMPLB & & PR I NT & TING & & TBST & D. - & 15 \\
\hline LAB & $\sqrt{67}$ & 76 o EAM & 3 PBR 8 & $\triangle R B M E$ & & $\mathbf{2 3 7}$ & 75 GRA & s PBR $\mathbf{s}$ & SQUARB & & & & \\
\hline $\operatorname{CODE}$ & MEAN & DEV & N. DBV & SDR & R. BDR & $M B A N$ & DBV & $\mathbf{N} \cdot \mathbf{D B V}$ & $\mathbf{S D R}$ & R. SDR & VAR & $\mathbf{F}$ & LAB \\
\hline 1121 & 15.33 & -1.53 & -1.29 & 1.03 & .88 & 25.20 & -2.52 & $-1 \cdot 34$ & 1.42 & .77 & $10 \mathrm{C}$ & $\boldsymbol{\theta}$ & 2121 \\
\hline L128 & 18.47 & 1.60 & $1 \cdot 35$ & .83 & .71 & 27.60 & -.12 & .007 & 1.80 & .97 & $10 \mathrm{C}$ & $\boldsymbol{\theta}$ & L128 \\
\hline LI31 & 13.13 & $-1 \cdot 73$ & $-1 \cdot 46$ & .35 & .30 & 25.73 & $-1 \cdot 99$ & -1.06 & .59 & .32 & $10 C$ & $\theta$ & 2131 \\
\hline L834 & 16.23 & -.63 & -.53 & .59 & .51 & 30.80 & 3.08 & 2.64 & 1.54 & .83 & $10 \mathrm{C}$ & $\star$ & 2134 \\
\hline L1 50 & 17.27 & .40 & .34 & 1.61 & 1.38 & 28.73 & 1.01 & .54 & $2 \cdot 23$ & 1.20 & $10 C$ & $\boldsymbol{\theta}$ & L150 \\
\hline L1 53 & 17.90 & 1.03 & .87 & 1.47 & 1.25 & 29.80 & 2.08 & 1.10 & 1.66 & .89 & $10 \mathrm{C}$ & $\boldsymbol{\sigma}$ & 2153 \\
\hline L158 & 16.20 & -.67 &.- .56 & 2.37 & 1.17 & 26.73 & -.99 &. .53 & 1.22 & .66 & $10 c$ & $\boldsymbol{\theta}$ & 2158 \\
\hline L1 61 & $12, \in 3$ & -4.23 & -3.56 & 1.11 & .95 & 24.33 & -3.39 & -1.80 & 2.23 & 1.20 & $10 \mathrm{C}$ & $x$ & 2161 \\
\hline L1 67 & 16.60 & -.27 & -.22 & .54 & .46 & $26 \cdot 45$ & $-1 \cdot 28$ & -.68 & .89 & .48 & $10 C$ & $\theta$ & 2167 \\
\hline 2183 & 17.03 & .17 & .14 & 1.34 & 1.15 & 27.83 & .11 & .06 & 2.03 & 1.09 & $10 \mathrm{C}$ & $\theta$ & 2183 \\
\hline L20 3B & 13.97 & $-2 \cdot 90$ & -2.44 & 1.25 & 1.07 & 28.90 & 1.18 & .63 & 3.10 & 1.67 & $10 \mathrm{C}$ & $x$ & L203B \\
\hline L2039 & 16.05 & -.82 & -.69 & 1.39 & 1.19 & 26.93 & .79 & -.42 & 3.16 & 1.70 & $10 \mathrm{C}$ & $\boldsymbol{\theta}$ & L203S \\
\hline 1207 & 18.53 & 1.67 & 1.40 & 1.01 & .86 & 30.80 & 3.08 & 1.61 & 2.26 & 1.22 & $10 \mathrm{C}$ & $\boldsymbol{\theta}$ & L207 \\
\hline 1212 & 16.77 & -010 & -.08 & 1.18 & 1.01 & 26.97 & .76 & -.40 & 1.83 & .98 & $10 \mathrm{C}$ & $\boldsymbol{\theta}$ & 1212 \\
\hline L223A & 19.17 & 2.31 & 1.94 & 1.58 & 1.35 & $31 \cdot 57$ & 3.84 & 2.04 & 2.02 & 1.09 & $10 \mathrm{C}$ & $\boldsymbol{\theta}$ & L223A \\
\hline L22 5 & 16.97 & .10 & .09 & 1.30 & 1.11 & 29.50 & 1.78 & .94 & 2.04 & 1.10 & $\$ 0 \mathrm{C}$ & $\theta$ & LE25 \\
\hline L23i & 15.73 & -1.13 & -.95 & $\$ .45$ & 1.24 & 27.97 & .24 & .13 & 1.32 & .82 & $10 \mathrm{C}$ & $\boldsymbol{\theta}$ & L2 32 \\
\hline $1237 A$ & 14.13 & $-2 \cdot 73$ & $-2 \cdot 30$ & .92 & .78 & 24.93 & -2.79 & $-1 \cdot 4.8$ & .80 & .43 & $\$ 0 C$ & $\boldsymbol{\theta}$ & $1237 A$ \\
\hline L237B & 17.00 & .13 & .11 & 1.07 & .91 & 26.53 & $-1 \cdot 19$ & -.63 & .88 & .47 & $10 C$ & $\theta$ & L237 B \\
\hline 1243 & $17 \cdot 33$ & .47 & .39 & 1.36 & 1.16 & 26.23 & $-1 \cdot 49$ & -.79 & 2.75 & 1.48 & $.80 \mathrm{C}$ & $\theta$ & 2243 \\
\hline L248 & $17 \cdot 42$ & .55 & .47 & 1.10 & . 94 & 27.19 & -.53 & -028 & 2.48 & .79 & $10 E$ & $\sigma$ & 2248 \\
\hline 1249 & 16.95 & .09 & .07 & $1 \cdot 36$ & 1.17 & 28.28 & .56 & . 30 & 2.01 & 1.08 & $10 \mathrm{C}$ & $\theta$ & 1249 \\
\hline 1261 & 16.60 & -.27 & -.22 & 1.00 & .86 & 27.10 & -.62 & -.33 & 2.01 & 1.08 & 100 & $\theta$ & L261 \\
\hline L264 & $16 \cdot 67$ & -.20 & -017 & .82 & .70 & 26.27 & $-1 \cdot 46$ & -.78 & 1.49 & .80 & $10 \mathrm{C}$ & $\boldsymbol{\theta}$ & L264 \\
\hline L274 & 17.07 & $\cdot 20$ & .17 & 1.00 & .85 & 30.63 & 2.91 & $1 \cdot 55$ & 1.39 & .75 & $10 C$ & $\boldsymbol{\theta}$ & L274 \\
\hline L278 & 15.07 & $-1 \cdot 80$ & $-1 \cdot 51$ & 1.33 & 1.14 & 26.63 & -1.09 &. .58 & 2.05 & 1.10 & $10 \mathrm{C}$ & $\boldsymbol{\theta}$ & L278 \\
\hline L279 & 16.83 & -.03 & -.03 & 1.03 & .88 & 25.93 & -1.79 & -.95 & 1.99 & 1.07 & $10 C$ & $\boldsymbol{\theta}$ & 2279 \\
\hline 1299 & 18.87 & 2.00 & 1.68 & 1.53 & 1.31 & 31.60 & 3.88 & 2.06 & 2.00 & 1.08 & $10 C$ & $\boldsymbol{\theta}$ & 2299 \\
\hline L311 & 18.03 & 1.17 & .98 & 1.41 & 1.20 & $31 \cdot 13$ & 3.41 & 1.81 & 2.49 & 1.34 & $10 \mathrm{C}$ & $\boldsymbol{\theta}$ & L311 \\
\hline L312 & 16.99 & .12 & .10 & 1.09 & .93 & 28.46 & .74 & .39 & 1.72 & .92 & $10 \mathrm{C}$ & $\boldsymbol{\theta}$ & L312 \\
\hline 1315 & 19.27 & 2.40 & 2.02 & 1.56 & 1.33 & 30.73 & 3.01 & 1.60 & 2.10 & 1.13 & $10 C$ & $\boldsymbol{\theta}$ & 2315 \\
\hline L321 & $17 \cdot 23$ & .37 & . 31 & 1.13 & .97 & 29.07 & 1.34 & .71 & 2.23 & 1.20 & $10 \mathrm{C}$ & $\boldsymbol{\theta}$ & L321 \\
\hline $13 \approx 6$ & 16.80 & -.07 & -.05 & 1.10 & .94 & 26.53 & -1.19 & -.63 & 1.43 & .77 & $\triangle O C$ & $\boldsymbol{\theta}$ & 2326 \\
\hline$L=36$ & 16.31 &. .56 & -.47 & 1.20 & 1.03 & 26.12 & -1.60 &. .85 & 2.79 & 1.50 & $10 C$ & $\theta$ & 2330 \\
\hline 4331 & 16.67 & .00 & .00 & 1.45 & 1.25 & 27.40 & -.32 & -.87 & 2.16 & 1.17 & $10 \mathrm{C}$ & $\boldsymbol{\theta}$ & L331 \\
\hline L333 & 14.75 & $-2 \cdot 11$ & -1.78 & 1.26 & 1.08 & 24.88 & $-2 \cdot 84$ & -1.51 & 2.16 & 1.16 & $10 C$ & 9 & 1333 \\
\hline 1339 & $10 \cdot 51$ & -6.35 & $-5 \cdot 34$ & .05 & .04 & $15 \cdot 52$ & $-12 \cdot 20$ & -6.49 & .11 & .06 & $10 C$ & $*$ & 1339 \\
\hline 1344 & $15 \cdot 57$ & $-1 \cdot 30$ & -1.09 & 1.22 & 1.05 & 26.80 & .992 & $=49$ & 2.12 & 1.14 & $10 \mathrm{C}$ & 6 & 2344 \\
\hline 1356 & $17 \cdot 43$ & .56 & .47 & 1.17 & 1.00 & 25.78 & -1.94 & -1.03 & 2.23 & 1.20 & 100 & $\theta$ & 2356 \\
\hline 1358 & 16.90 & .03 & .03 & .51 & .43 & 28.19 & -47 & .25 & 1.39 & .75 & $10 \mathrm{C}$ & $\boldsymbol{\theta}$ & L358 \\
\hline$L 360$ & $17 \cdot 52$ & .65 & .55 & 1.11 & . 95 & 27.65 & .008 & -.04 & 1.81 & .98 & $10 \mathrm{C}$ & $\boldsymbol{\sigma}$ & 2360 \\
\hline L366 & $17 \cdot 60$ & .73 & .62 & 8.43 & $1 \cdot 22$ & 27.23 & -.49 & -.26 & 1.92 & 1.03 & $10 \mathrm{C}$ & $\boldsymbol{\theta}$ & L366 \\
\hline 1390 & 18.33 & 1.47 & 1.23 & $1 \cdot 36$ & 1.16 & 28.60 & .88 & .47 & 1.20 & .65 & $10 C$ & $\theta$ & L390 \\
\hline 2568 & $17 \cdot 50$ & .63 & .53 & .96 & .82 & $28 \cdot 32$ & .60 & $\cdot 32$ & 2.35 & 1.37 & $10 C$ & $\boldsymbol{\theta}$ & 2568 \\
\hline 1599 & $17 \cdot 51$ & .63 & .55 & .95 & .81 & 28.25 & .52 & .28 & 1.89 & 1.02 & $10 \mathrm{C}$ & $\theta$ & L599 \\
\hline 1626 & 15.68 & $-1 \cdot 18$ & -.99 & 1.03 & .88 & 25.85 & $-1 \cdot 88$ & -1.00 & 2.19 & 1.18 & $10 \mathrm{C}$ & $\theta$ & 1626 \\
\hline LS 84 & 14.45 & $-2 \cdot 42$ & -2.03 & 1.33 & 1.14 & 24.91 & $-2 \cdot 82$ & -1.50 & 1.97 & 1.06 & $10 \mathrm{C}$ & $\theta$ & 2684 \\
\hline
\end{tabular}

GR. MBAN - $16.87 \mathrm{PSI}$

GRAND LBAN - $27.72 \mathrm{PBI}$

AD OP KRANE $.1 .88 \mathrm{PBI}$

SD MBANS - 1.19 P8

$\triangle V E R A O B$ GD - 1.17 PEI

TBST DBTBRMINATIENS - IS 44 LABS IN ORAND MEANS GR. MBAN - 116.3 EILEPAECNL

\begin{tabular}{|c|c|c|c|c|c|c|c|c|c|c|c|c|}
\hline $\begin{array}{l}1219 \\
1242\end{array}$ & $\begin{array}{l}15.82 \\
19.43\end{array}$ & $\begin{array}{r}-1.04 \\
2.56\end{array}$ & $\begin{array}{l}-0.80 \\
2.16\end{array}$ & $\begin{array}{l}1.60 \\
1.57\end{array}$ & $\begin{array}{l}1.37 \\
1.34\end{array}$ & $\begin{array}{l}25.96 \\
29.88\end{array}$ & $\begin{array}{r}-1.77 \\
2.16\end{array}$ & $\begin{array}{l}-.94 \\
1.15\end{array}$ & $\begin{array}{l}2.37 \\
2.23\end{array}$ & $\begin{array}{l}1.28 \\
1.20\end{array}$ & $\begin{array}{l}10 T \\
10 T\end{array}$ & $\begin{array}{l}\text { - LE19 } \\
\text { - L242 }\end{array}$ \\
\hline$L 250 \mathrm{~L}$ & $17 \cdot 50$ & .63 & .53 & $1 \cdot 31$ & 1.12 & 26.78 &. .95 & -.50 & 2.17 & 1.17 & $10 N$ & - L250L \\
\hline 1269 & 20.73 & 3.87 & 3.25 & 1.62 & 1.39 & 31.40 & 3.68 & 1.96 & 2.86 & 1.17 & 104 & - 1269 \\
\hline 2484 & 15.83 & -1.03 & -.87 & 1.13 & .97 & $25 \cdot 13$ & -2.59 & -1.38 & 1.58 & .85 & IOM & - 2484 \\
\hline
\end{tabular}

TETAL NOMBER OP LABERATERIBE PBPERTINO 52 "

Best values: $\mathrm{J} 6716.9+2.2 \mathrm{ps} 1$

K37 27.5 $\pm 3.0 \mathrm{ps}$

The following laboratorles were omitted from the

grand means because of extreme test results: 339. 
BURSTINO STRBNGTE, PSI

TAPPI SIANDARD T403 6S-76, BURSTINO STRBNOTB OF PAPBR - PBRIINS MODEL C

\begin{tabular}{|c|c|c|c|c|c|c|c|c|c|c|c|c|c|c|c|c|c|}
\hline \multirow{2}{*}{$\begin{array}{l}\mathrm{LAB} \\
\mathrm{C} \in \mathrm{DE}\end{array}$} & \multirow[b]{2}{*}{$\mathbf{F}$} & \multicolumn{2}{|c|}{ MEANS } & \multicolumn{2}{|c|}{ CEURDINATBS } & \multirow{2}{*}{$\begin{array}{l}\text { A Vo } \\
\text { R. SDR }\end{array}$} & \multirow[b]{2}{*}{ VAR } & \multirow{2}{*}{\multicolumn{2}{|c|}{ PREPERTY $=-$ TBST }} & \multirow{2}{*}{\multicolumn{6}{|c|}{ INSI R UMBNT --- CENDITIENS }} & \multirow[b]{3}{*}{ MANUAL } & \multirow[b]{3}{*}{ CLAMP } \\
\hline & & J67 & 137 & MAJUR & YINOR & & & & & & & & & & & & \\
\hline 1339 & 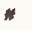 & 10.51 & 15.52 & $-13 \cdot 76$ &. .24 & .05 & $10 \mathrm{C}$ & BURST ING & STRBNGTB & UP & Io & 45 & PSI. & PBRIINS & c. & & \\
\hline L161 & $\mathbf{x}$ & 12.63 & $24 \cdot 33$ & -5.00 & 2.10 & 1.08 & $10 \mathrm{C}$ & BURSTINO & STRBNOTB & UP & Io & 45 & PSI. & PBRIINS & c. & CANUAL & CLAMP \\
\hline $2203 B$ & $\mathbf{x}$ & 13.97 & 28.90 & $\ldots 35$ & 3.11 & 1.37 & $10 \mathrm{C}$ & BURSTINO & STRBNGTB & UP & IO & 45 & PSI. & PERIINS & c. & KANUAL & CLAMP \\
\hline L237A & $\theta$ & 14.13 & 24.93 & -3.76 & 1.07 & .61 & $10 \mathrm{C}$ & BURSIING & STRBNOTB & UP & To & 45 & PSI. & PEREINS & c. & JUNUAL & CLAXP \\
\hline 2684 & $\theta$ & 14.45 & 24.91 & -3.63 & .78 & 1.10 & $10 \mathrm{C}$ & BURSIINO & STRBNGTH & UP & Tө & 45 & PSI. & PBRIINS & c. & VANUAL & CLAXP \\
\hline 1333 & 0 & 14.75 & 24.88 & -3.51 & .50 & 1.12 & $10 \mathrm{C}$ & BURST ING & STRBNGTE & UP & $T 0$ & 45 & PSI. & PBREINS & c, & MANUAL & CLAXP \\
\hline 1278 & $\theta$ & $\$ 5.07$ & $26 \cdot 63$ & $-1 \cdot 82$ & 1.06 & 1.12 & $10 \mathrm{C}$ & BURSTING & BTRENGTE & UP & To & 45 & PSI. & PBREINS & c. & MANUAL & CLAMP \\
\hline L138 & $\theta$ & 15.23 & 25.73 & -2.58 & .57 & .31 & $10 \mathrm{C}$ & BURSTINO & STRBNGTE & UP & $T \theta$ & 45 & PSI. & PBRIINS & c. & MANUAL & CLAMP \\
\hline LI 28 & $\theta$ & 15.33 & 25.20 & -2.95 & .14 & - 82 & $10 c$ & BURETINO & STRENOTH & UP & To & 45 & PSI. & PBREINS & c. & KANUAL & CLAKP \\
\hline 2344 & $\theta$ & 15.57 & 26.80 & $-1 \cdot 43$ & .70 & 1.09 & $10 c$ & BURSTINO & STRENOTE & UP & $I \theta$ & 45 & PSI. & PEREINS & c. & MANUAL & CLAMP \\
\hline 2626 & б & 15.68 & 25.85 & -2.21 & .14 & 1.03 & $10 \mathrm{C}$ & BURSTINO & STRBNOTB & UP & Te & 45 & PSI. & PBREINS & c. & MANUAC & CLAMP \\
\hline 1232 & $\sigma$ & 15.73 & 27.97 & -.33 & 1.11 & 1.03 & $10 \mathrm{C}$ & BURSTINO & 8TRBNOTH & $\mathbf{U P}$ & TE & 45 & PQI. & PB RIINA & c. & MANUAL & CLAMP \\
\hline 1219 & $\cdot$ & $\$ 5.82$ & $25 \cdot 56$ & -2.05 & .08 & 1.32 & $10 \mathrm{~T}$ & BURATINO & STRBNGTB & UP & To & 45 & PSI. & $I * T, M A N U$ & OAL $c$ & CLAMP & \\
\hline 2484 & $\bullet$ & 85.83 & 25.13 & -2.77 & -.33 & .91 & $10 y$ & BURSTINO & STRBNGTB & UP & To & 45 & PQI, & RBOLBD $M T$ & T/MOI & IT, KANU AL & L CLAMP \\
\hline L2039 & $\theta$ & 26.05 & 26.93 & -1.08 & .34 & 1.44 & $10 \mathrm{C}$ & BURSTINO & STRBNOTB & UP & To & 45 & PSI. & PBRIINS & c. & MANUAL & CLAMP \\
\hline L158 & ฮ & 16.20 & 26.73 & $-1 \cdot 19$ & .11 & .92 & $10 \mathrm{C}$ & BURSTINO & STRBNOTE & UP & To & 45 & PQI, & PBRIINS & c. & MANOAL & CLAMP \\
\hline 2834 & 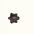 & 86.23 & 30.80 & 2.40 & 2.02 & .67 & $10 \mathrm{C}$ & BURSTINO & STRENOTE & UP & To & 45 & PSI. & PBRIINS & c. & LANUAL & CLAMP \\
\hline$L 330$ & $\theta$ & 16.31 & 26.12 & -1.68 &. .27 & 1.27 & $10 \mathrm{C}$ & BURSIINO & STRBNGTB & UP & To & 45 & PSI. & PBREINS & c. & MANUAL & CLAMP \\
\hline L867 & $\theta$ & 86.60 & 26.45 & -1.25 & -.38 & .47 & $10 \mathrm{C}$ & BURSTINO & STRBNOTB & UP & IO & 45 & PSI. & PBRIINS & c. & KANUAL & CLAMP \\
\hline L268 & $\boldsymbol{\theta}$ & 16.60 & $27 \cdot 10$ &. .67 & .006 & .97 & $10 \mathrm{C}$ & BURSTINO & STRBNOTB & UP & IO & 45 & PSI. & PBRIINS & c. & MANUAL & CLAMP \\
\hline 1264 & 0 & 16.67 & $26 \cdot 27$ & $-1 \cdot 38$ &. .52 & .75 & $10 \mathrm{C}$ & BURSIINO & STRBNOTB & UP & To & 45 & PSI. & PBRIINS & c. & YANUAL & CLAMP \\
\hline L2\& 2 & $\theta$ & 16.77 & 26.97 & -.71 &. .27 & 1.00 & $10 \mathrm{C}$ & BURETING & STRBNOTB & UP & To & 45 & P\&I. & PBRIINS & c. & MANUAL & CIAMP \\
\hline$L 326$ & $\theta$ & 16.80 & 26.53 & -1.08 &. .51 & .86 & $10 \mathrm{C}$ & BURSTINO & STRBNOTB & UP & To & 45 & PEI, & PBRIINS & c. & MANUAL & CLAMP \\
\hline 1279 & $\theta$ & 16.83 & 25.93 & -1.59 & .83 & .98 & $10 \mathrm{C}$ & BURSTING & STRENOTB & UP & To & 45 & PQI, & PBRIINS & c. & MANUAL & CLAMP \\
\hline L233 & $\theta$ & 16.87 & $27 \cdot 40$ & -.28 &. .16 & 1.21 & $10 \mathrm{C}$ & BURSTING & STRBNGTE & UP & TE & 45 & PSI. & PB REI NS & c. & MANUAL & CLAMP \\
\hline L358 & $\boldsymbol{\sigma}$ & 16.90 & $28 \cdot 19$ & .43 & .19 & .59 & 100 & BURSTINO & ST RBNGTB & UP & $\mathbf{T \theta}$ & 45 & PSI. & PBRIINS & c. & MANUAL & CLAYP \\
\hline 2249 & $\sigma$ & 26.95 & 28.28 & .53 & .19 & 1.12 & $10 \mathrm{C}$ & BURSTINO & STRBNGTB & UP & T6 & 45 & PSI. & FBREINS & c. & MANUAL & CLAMP \\
\hline L225 & ฮ & 86.97 & 29.50 & 1.68 & .76 & 1.11 & $10 \mathrm{C}$ & BURSTINO & STRBNGTH & UP & To & 45 & PSI. & PBRIINS & c. & MANOAL & CLAKP \\
\hline L382 & $\theta$ & 16.99 & 28.46 & .70 & .24 & .93 & $10 \mathrm{C}$ & BURSTINO & STRBNOTH & UP & IO & 45 & PSI. & PBRIINS & c. & KANUAL & CLAKP \\
\hline L237B & 0 & 17.00 & $26 \cdot 53$ & -.98 & .69 & .69 & $10 \mathrm{C}$ & BURSTINO & BTRBNGTH & UP & To & 45 & PSI, & PBRIINS & c. & MANUAL & CLAYP \\
\hline 2183 & 0 & 17.03 & 27.83 & .18 & .10 & 1.12 & $10 \mathrm{C}$ & BURSTINO & SIRENGTB & UP & T & 45 & PSI, & PBRIINS & c. & MANUAL & CLAMP \\
\hline 1274 & 0 & 17.07 & 30.63 & 2.65 & 1.21 & .80 & $10 \mathrm{C}$ & BURSTINO & STRBNOTE & UP & To & 45 & PSI, & PERIINS & c. & MANUAL & CLAMP \\
\hline 2321 & $\theta$ & 17.23 & 29.07 & 1.36 & .32 & 1.08 & $10 \mathrm{C}$ & BUREIINO & STRBNGTE & UP & To & 45 & PSI. & PBRIINS & c. & MANUAL & CLAMP \\
\hline 2150 & $\theta$ & 17.27 & 28.73 & 1.08 & .13 & 1.29 & $10 \mathrm{C}$ & BURSTINO & STRENGTE & UP & To & 45 & P\&I. & PERIINS & c. & MANUAL & CLAMP \\
\hline$L 243$ & $\theta$ & 17.33 & 26.23 & -1.09 & -1.12 & $1 \cdot 32$ & $10 \mathrm{c}$ & BURSTINO & ATRENOTE & UP & Io & 45 & PSI. & PBRIINS & $c$ & MANUAL & CLAMP \\
\hline L2 48 & 0 & 17.42 & 27.19 & -.20 & .074 & .87 & $10 B$ & BURETINO & STRBNGTB & UP & Io & 45 & PSI. & PBRIINS & c. & MANUAL & CLAMP \\
\hline$L 356$ & $\theta$ & 17.43 & 25.78 & $-1 \cdot 44$ & -1.42 & 1.10 & $10 \mathrm{C}$ & BURSTINO & STRBNOTB & UP & I $\sigma$ & 45 & PSI. & PBREINS & c. & MANUAL & CLAXP \\
\hline 22501 & - & 17.50 & 26.78 & -.53 & -1.01 & 1.14 & $10 \mathrm{~N}$ & BURSTINO & STRENOTE & UP & To & 45 & PSI, I & LEXUROY, & - MAN. & I. CLAMP. & $20 \mathrm{C}, 65$ \\
\hline L568 & $\theta$ & 17.50 & $28 \cdot 32$ & .83 &. .27 & 1.10 & $10 \mathrm{C}$ & BURSTINO & STRBNOTH & UP & To & 45 & PEI. & PBRIINS & c. & KANUAL & CLAMP \\
\hline 1599 & $\theta$ & 17.51 & 28.25 & .77 &. .32 & .91 & $10 \mathrm{C}$ & BURSTING & STRENOTE & UP & To & 45 & PQI. & PBRIINS & c. & KANUAL & CLAMP \\
\hline 2360 & $\sigma$ & 17.52 & 27.65 & .24 & .061 & .96 & $10 \mathrm{C}$ & BU RSTINO & STRBNOTE & UP & To & 45 & PSI. & PBRIINS & c. & MANUAL & CLAXP \\
\hline 2366 & $\theta$ & 17.60 & $27 \cdot 23$ & -.08 &. .88 & 1.13 & $10 \mathrm{C}$ & BURBIINO & BTRENOTE & UP & Io & 45 & PSI. & PBRIINS & c. & MANUAL & CLAMP \\
\hline 2153 & $\theta$ & 17.90 & 29.80 & 2.32 & .08 & 1.07 & $10 \mathrm{C}$ & BURGTINO & STRBNGTE & OP & To & 45 & PSI. & PBRIINS & c. & MANUAL & CLAYP \\
\hline 2311 & $\boldsymbol{\sigma}$ & 18.03 & 31.13 & 3.55 & .60 & 1.27 & $10 \mathrm{C}$ & BURST INO & STRBNOTE & UP & To & 45 & PQI. & PERIINS & c. & MANUAL & CLAXP \\
\hline 2390 & $\sigma$ & 18.33 & 28.60 & 1.47 & -.87 & .90 & $10 \mathrm{C}$ & BURSTINO & STRENGTE & UP & To & 45 & PQI. & PBRIIN & c. & KANUAL & CLAMP \\
\hline 2128 & $\boldsymbol{\theta}$ & 18.47 & 27.60 & .66 & -1.47 & .84 & $10 \mathrm{C}$ & BURETING & STRBNंOTB & U P & To & 45 & PQI, & PBRIINS & c. & MUNOAL & CLAMP \\
\hline 1207 & $\theta$ & 18.53 & 30.80 & 3.50 & .00 & 1.04 & $10 \mathrm{C}$ & BURAIINO & STRBNOTE & UP & To & 45 & PSI. & PBRIINS & c. & MANUAL & CLAMP \\
\hline$L 299$ & 6 & 18.87 & 31.60 & 4.36 & .09 & 1.19 & $10 \mathrm{C}$ & BURSTINO & STRENOTE & UP & To & 45 & PSI. & PBRIINS & c. & MNUAL & CLAMP \\
\hline L223A & $\sigma$ & 19.17 & 31.57 & 4.48 &. .19 & 1.22 & $10 \mathrm{C}$ & BURETINO & STRENOTE & UP & To & 45 & PSI. & PBRIINS & c. & MANUAL & CLAMP \\
\hline$L 315$ & $\sigma$ & 19.27 & 30.73 & 3.79 & .67 & 1.23 & $10 \mathrm{C}$ & BURSTING & STRBNOTE & UP & To & 45 & P\&I. & PBREINS & c. & MUNUAL & CLAMP \\
\hline L242 & - & 19.43 & 29.88 & 3.12 & $-1 \cdot 23$ & 1.27 & $10 T$ & BURATINO & STRBNOTE & UP & To & 45 & 200 & 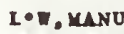 & UAL & CLAXP & \\
\hline LE 69 & - & 20.73 & $31 \cdot 40$ & 5.08 & -1.64 & 1.28 & 104 & BURSIING & STRENOTE & UP & IO & 45 & PSI. & PBRIINS & $\boldsymbol{\Lambda}$ & KANUAL & CLAMP \\
\hline BMAP & & $\begin{array}{c}16.87 \\
95 \%\end{array}$ & $\begin{array}{l}27,72 \\
\text { LLI PSB : }\end{array}$ & 5.40 & 1.86 & $\begin{array}{l}1.00 \\
\text { IT B }\end{array}$ & GAMM & $M A-61$ & BS & & & & & & & & \\
\hline
\end{tabular}



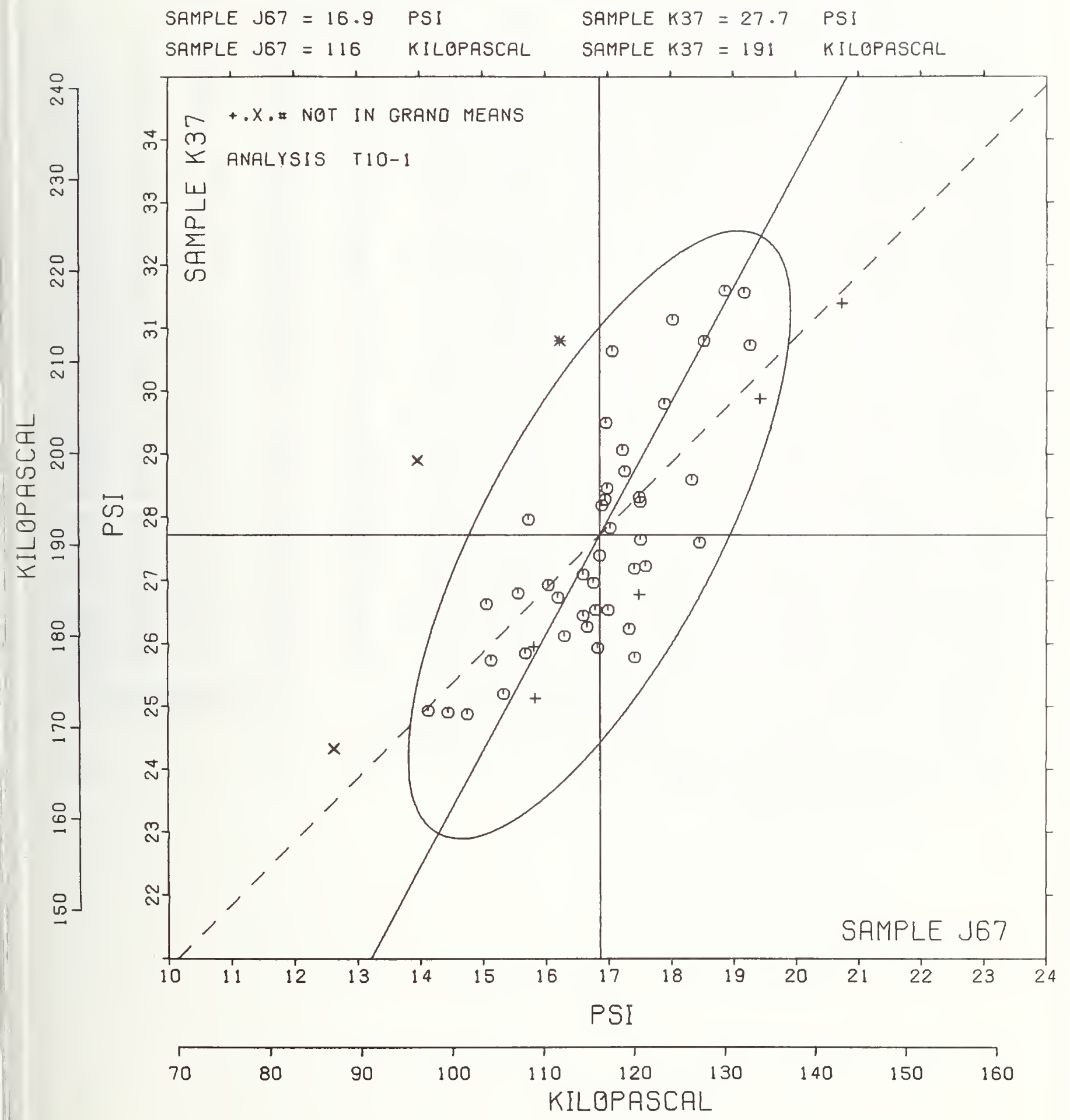
TAPPI STANDARD T403 GS-76. BURSING STRBNGTH OF PAPBR - PBRIINS MODEL C-A OR C

\begin{tabular}{|c|c|c|c|c|c|c|c|c|c|c|c|c|c|}
\hline \multirow{3}{*}{$\begin{array}{l}\mathrm{L} \triangle \mathrm{B} \\
\mathrm{C} D \mathrm{DB}\end{array}$} & \multirow{3}{*}{$\begin{array}{c}\text { SAMPLB } \\
\text { J67 } \\
\text { MBAN }\end{array}$} & \multicolumn{4}{|c|}{ PRINIINO } & \multirow{3}{*}{$\begin{array}{c}\text { SAMPLE } \\
\text { B37 } \\
\text { MEAN }\end{array}$} & \multicolumn{4}{|c|}{ PRINIINO } & \multirow{3}{*}{$\begin{array}{r}\text { TEST } \\
\text { VAR }\end{array}$} & \multirow{3}{*}{$\begin{array}{c}D_{\bullet}= \\
F\end{array}$} & \multirow{3}{*}{$\begin{array}{l}15 \\
\text { LAB }\end{array}$} \\
\hline & & 76 OFAH & 9 PBR 5 & $\triangle R B \quad M E$ & & & 75 GR & S PBR & $\triangle R B$ & & & & \\
\hline & & DEV & N. DBV & SDR & R. SDR & & DBV & $\mathrm{N} \cdot \mathrm{DEV}$ & SDR & R. SDR & & & \\
\hline L1 00 & 18.17 & 1.33 & $\bullet 77$ & .91 & .85 & $27 \cdot 40$ &. .03 & .01 & .99 & .54 & $10 \mathrm{D}$ & $\theta$ & 2100 \\
\hline L8 05 & 13.83 & -3.00 & $-1 \cdot 72$ & .72 & .67 & $22 \cdot 81$ & -4.61 & -1.94 & 2.54 & 1.39 & $10 \mathrm{D}$ & $\theta$ & L105 \\
\hline 1122 & 16.80 & .0 .03 & -.02 & 1.01 & .94 & 26.00 & $-1 \cdot 43$ & -.60 & 2.07 & 1.23 & $10 F$ & $\theta$ & L1 22 \\
\hline L1 25 & 18.60 & 2.77 & 1.01 & 1.45 & 1.35 & 30.37 & 2.94 & 1.24 & 1.37 & .75 & $10 \mathrm{D}$ & $\theta$ & L125 \\
\hline L\$ 48 & 17.53 & .70 & .40 & 1.26 & 1.17 & 28.23 & .81 & .34 & 1.31 & .71 & 100 & $\theta$ & 2141 \\
\hline 1148 & 17.80 & .97 & .56 & 1.21 & 1.12 & 28.80 & 1.37 & .58 & 1.90 & 1.03 & $10 D$ & $\theta$ & 2148 \\
\hline L8 57 & 18.53 & 1.70 & .98 & 1.39 & 1.29 & 29.77 & 2.34 & .98 & 2.76 & 1.50 & 100 & $\theta$ & L157 \\
\hline LI 62 & 16.47 & -.37 & -.21 & 1.19 & 1.10 & 25.73 & -1.69 & -071 & 1.94 & 1.06 & 100 & $\theta$ & L162 \\
\hline 2863 & 16.10 & -.73 & -.42 & 1.02 & .95 & 26.30 & -1.13 & -.47 & 1.92 & 1.04 & 100 & $\theta$ & 2163 \\
\hline 1166 & 17.63 & .80 & .46 & 1.13 & 1.04 & 29.83 & 2.41 & 1.01 & 1.83 & 1.00 & $10 \mathrm{D}$ & $\boldsymbol{\theta}$ & L1 66 \\
\hline L1 85 & 18.53 & 1.70 & .98 & 1.19 & 1.10 & 29.47 & 2.04 & .86 & 1.73 & .94 & $10 \mathrm{D}$ & $\boldsymbol{\theta}$ & 2185 \\
\hline L190C & 16,63 & -.20 &. .11 & .74 & .69 & $25 \cdot 17$ & -2.26 & -.95 & 1.90 & 1.03 & $10 \mathrm{D}$ & $\theta$ & L190 \\
\hline L190R & 17.13 & .30 & .17 & 1.17 & 1.09 & 28.23 & .81 & .34 & 1.67 & .91 & $10 D$ & 6 & 2190 \\
\hline L1 94 & $18 \cdot 36$ & 1.53 & .88 & .71 & .66 & 27.53 & .10 & .04 & 1.24 & .68 & $10 \mathrm{D}$ & $\boldsymbol{\theta}$ & L1 94 \\
\hline L28 7 & 17.27 & .43 & .25 & .70 & .65 & 25.53 & -1.89 & -080 & 1.30 & .71 & $10 F$ & $\theta$ & 2217 \\
\hline L22 68 & 17.27 & .44 & .25 & 1.41 & 1.31 & 28.05 & .62 & .26 & 1.75 & .95 & 100 & $\theta$ & $L=26$ \\
\hline LE2 $6 \mathrm{C}$ & 18.53 & 1.69 & .97 & .71 & .66 & 29.07 & 1.64 & .69 & 2.28 & 1.24 & 100 & $\theta$ & LE 26 \\
\hline 1241 & 18.17 & 1.33 & .77 & .99 & .91 & 29.47 & 2.04 & .86 & 1.52 & .83 & $10 \mathrm{D}$ & $\boldsymbol{\theta}$ & L241 \\
\hline$L 255$ & 16.27 & -.57 & -.32 & .70 & .65 & 25.47 & -1.96 & -.82 & .74 & .40 & $10 \mathrm{D}$ & $\theta$ & 1255 \\
\hline L257A & 17.67 & .83 & .48 & .90 & .83 & 28.67 & 1.24 & .52 & 1.72 & .94 & $10 \mathrm{D}$ & $\theta$ & L257 \\
\hline L2578 & 17.60 & .77 & .44 & 1.06 & .98 & 27.47 & .04 & .02 & 1.92 & 1.05 & $10 D$ & $\boldsymbol{\theta}$ & L257 \\
\hline L257C & 17.67 & .83 & .48 & 1.45 & 1.34 & 28.27 & .84 & .35 & 1.58 & .86 & $10 \mathrm{D}$ & 0 & L2 57 \\
\hline L. 262 & $17 \cdot 27$ & .43 & .25 & 1.15 & 1.06 & 31.23 & 3.81 & 1.60 & 1.50 & .82 & 100 & $\sigma$ & 1262 \\
\hline 2275 & $12 \cdot 37$ & -4.46 & -2.56 & 1.13 & 1.05 & 22.96 & -4.47 & -1.88 & 2.74 & 1.49 & 100 & $\sigma$ & L2 75 \\
\hline L2 80 & 19.06 & 2.23 & 1.28 & 1.18 & 1.09 & 29.93 & 2.50 & 1.05 & 2.80 & 1.52 & $10 D$ & $\theta$ & L2 80 \\
\hline L285 & 15.73 & -1.10 &. .63 & 2.40 & 2.23 & 30.20 & 2.77 & 1.17 & 1.82 & .99 & $10 D$ & $\cdot$ & L285 \\
\hline 1309 & 16.89 & .06 & .04 & .67 & .62 & 27.87 & .45 & .19 & 2.28 & 1.24 & $10 D$ & $\theta$ & 1309 \\
\hline L35E & $15 \cdot 48$ & $-1 \cdot 35$ & -078 & .78 & .72 & 25.00 & $-2 \cdot 43$ & -1.02 & 1.95 & 1.06 & $10 D$ & $\theta$ & L.352 \\
\hline 1563 & 12.93 & -3.90 & $-2 \cdot 24$ & 1.35 & 1.25 & $21 \cdot 25$ & -6.17 & -2.59 & 1.68 & .91 & $10 \mathrm{D}$ & $*$ & 2563 \\
\hline 2567 & 14.67 & -2.17 & -1.24 & 1.45 & 1.34 & $25 \cdot 13$ & -2.29 & -.96 & 1.36 & .74 & $10 \mathrm{D}$ & $\sigma$ & L567 \\
\hline 1575 & 18.63 & 1.80 & 1.03 & 1.29 & 1.20 & 28.80 & 1.37 & .58 & 2.06 & 1.12 & 100 & 0 & L575 \\
\hline 1581 & 15.40 & $-1 \cdot 43$ & -.82 & 1. 87 & 1.74 & 27.50 & .07 & .03 & 1.63 & .89 & $10 \mathrm{D}$ & $\sigma$ & 2581 \\
\hline 1587 & $17 \cdot 20$ & .37 & .21 & .84 & .78 & 29.03 & 1.61 & .68 & 1.49 & .81 & $10 \mathrm{D}$ & $\boldsymbol{\theta}$ & L587 \\
\hline L652 & 12.83 & -4.00 & $-2 \cdot 30$ & 1.08 & 1.00 & 24.20 & -3.23 & -1.36 & 2.74 & 1.49 & $10 D$ & 0 & 1652 \\
\hline 2688 & 18.09 & 1.25 & .72 & 1.13 & 1.05 & 29.19 & 1.76 & .74 & 2.07 & 1.13 & $10 D$ & 0 & L688 \\
\hline
\end{tabular}

GR. MEAN $=16.83 \mathrm{PSI}$

GRAND UBAN - 27.43 PBI

TBST DBTBRMINATIENS - 15 SD MEANS - 1.74 PSI SD OP MBANB - $2,38 \mathrm{PBI}$ $\triangle V B R A O B$ SDR $=1.08 \mathrm{PSI}$ ORAND MEAN - 189.1 IILOPASCAI 


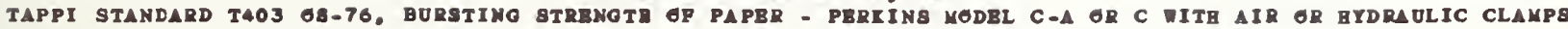

$\begin{array}{lll}\text { CAB } & \text { MBANS }\end{array}$

$1275 \quad 12.37 \quad 22.96$

L652 $12.83 \quad 24.20$

L313 $12.85 \quad 22.01$

L563 12.93 21.28

L105 13.83 22.81

$\begin{array}{llll}1567 & 6 & 14.67 \quad 25.13\end{array}$

$\begin{array}{llll}2581 & 15.40 \quad 27.50\end{array}$

1352615.4825 .00

L285 15.7330 .20

463.16 .1026 .30

L255 $16.27 \quad 25.47$

I1 $62016.47 \quad 25.73$

$1900 \cdot 16.63 \quad 25.17$

$2122,16.8026 .00$

1309616.8927 .87

$490 R \quad 17.13 \quad 28.23$

1587 17.20 29.03

$\begin{array}{llll}1262 & 17.27 & 31.23\end{array}$

$1217017.27 \quad 25.53$

L226B $17.27 \quad 28.05$

L141 $17.53 \quad 28.23$

L257B $17.60 \quad 27.47$

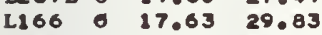

$\begin{array}{llll}257 C & 17.67 \quad 28.27\end{array}$

$12574017.67 \quad 28.67$

$2148 \quad 17.80 \quad 28.80$

L688 18.09 29.19

L100 $18.17 \quad 27.40$

$1241018.17 \quad 29.47$

1194 18.36 27.53

L226C 18.5329 .07

$\begin{array}{llll}2157 & 18.53 & 29.77\end{array}$

1185 ब $18.53 \quad 29.47$

4125 18.60 30.37

$1575 \quad 18.63 \quad 28.80$

$1280 \quad 19.06 \quad 29.93$

ONBANS: $16.83 \quad 27.43$

95* BLIIPAE:
CoERIMTES AVo

MAJGE MINGR R. ADR VAR PRGPBRTY--DBST INBTRUMBNT-D-CENDITIGNS

$-6.201 .16$

$-4.921 .48$

$-6.71 \quad .23$

$-7.30 \quad-.27$

$-5.50 \quad-.13$

$-3.12 .48$

0751.28

$-2.77 .-26$

$1.67 \quad 2.47$

$-1.34 \quad-.03$

$-1.94 \quad-.64$

$-1.60 \quad-.65$

$-1.98 \quad-1.11$

$-1.20 \quad-78$

.40 .20

84

1. 53

3.39

.21

.60

1.79

$-1.32-1.43$

.01

$1.06 \quad 012$

$.47 \quad-.68$

-.61
.70

.70
-.21

$1.16 \quad-2$

1.50

.01

$1.68 \quad-002$

$2.16 \quad-00$

$.73-1.12$

$2.44 \quad .05$

$.95-1.20$

$2.31 \quad .047$

$2.89 \quad-.08$

$2.64-0.25$

3.43 .20

$2.15 \quad-.71$

1.27 10D BURATINo STRBMGTE UP TO 45 PSI. PBRKINS CA OR C. AIR CLAMP

$1.2510 D$ BURATINO ATRENOTH UP TO 45 PSI. PBRIINB CA OR C, AIR CLAMP

Q1 101 BURATINO BTRENOTE OP TO 45 PQI, PBREINS CA OR C AIR CLAMP

1.08 10D BURETINO BTRBNOTE UP TO 45 PQI, PEREINS CA OR C $\triangle I R$ CLAMP

1.03 10 DURGTINe aTRBNOTE UP TO 45 P8I. PBRIINS CA OR C, $\triangle I R$ CLAMP

1.04 $10 \mathrm{D}$ BURATINO STRBMOTH UP TO 45 PSI, PBREINS CA OR C, AIR CLAMP 1.31 1OD BURETINO ATRENOTH UP TO 45 PBI. PBREINS CA OR C, $\triangle I R$ CLAMP . $8910 \mathrm{D}$ BURATINO ATRBNOTH UP TO 45 PSI. PBRINS CA OR C. AIR CLAMP 1.61 10D BURETINO ATRBNOTE UP TO 45 P8I. PBRIINS CA OR C, $\triangle I R$ CLAMP 1.00 $10 \mathrm{D}$ BURATINO BTRBNOTE UP TO 45 PBI. PBREINS CA GR C. AIR CLAMP

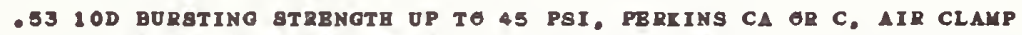
$1.0810 D$ BURATINO STRBNOTB UP TO 45 PQI, PBREINS CA GR C, AIR CLAKP .6 $10 D$ BORATINO ATRENOTE UP TO 45 PQI. PBREINS CA OR C AIR CLAMP 1.03 10 BURATINO ATRBNOTB UP TO 48 PaI, PBRINS C, H.CLAMP.TRANSDUCBR .93100 BURATINo 8TEBNOTE UP TG 45 PaI. PBRINS CA OR C. AIR CLAMP

1.00 10D BURATNO 8TRENOTE UP TO 45 PAI. PBREINS CA OR C. AIR CLAMP $.8010 D$ BURATINO BTRBNOTH UP TO 45 PQI. PBRIINS CA OR C, AIR CLAMP $.9410 D$ BURSTINO STRBNOTE UP TO 45 PSI. PBREINS CA OR C, $\triangle I R$ CLAMP .68 $10 F$ BURETINO ATRBNOTB UP TO 45 PSI.PBREINS C, H.CLNMP T RANADUCER 1. $1310 D$ BORATINO BTRENOTE UP TO 45 PSI. PHRINS CA OR C, AIR CLAMP

.94 10D BURATING ATRBNGTB UP TO 45 PSI. PBRIINS CA OR C AIR CLAMP $1.0110 D$ BURAT 1 NO 8TREHOTH UP TO 45 PQI. PBREINS CA OR C. AIR CLAMP $1.0210 \mathrm{D}$ BURATINO BTRENOTB UP TO 45 PSI. PBREINS CA OR C, $\triangle I R$ CLAMP $1.1010 D$ BURATINO BTRBNOTH UP TO 4 PSI. PBRINS CA OR C. AIR CLAMP $.8910 D$ BURETINO ATRBNGTE OP TO 48 PAI, PBRIINS CA OR C, $\triangle I R$ CLAMP

1.08 $10 D$ BURATINO ATRENOTB UP TO 45 PSI. PBRIINS CA OR C. AIR CLAMP $1.0910 D$ BURATINO ATRENOTE UP TO AS PSI, PBRIINS CA OR C, AIR CLAMP $.6910 \mathrm{D}$ BUROTINO 8TRBNOTE UP TO 45 P8I. PBREINS CA OR C. AIR CLAIP $.8710 D$ BURATINO ATRBNOTH UP TO 45 PQI, PERIINS CA OR C, AIR CLAMP $.6710 D$ BURSTINO aTRBNOTH OP TO 45 PSI. PBREINS CA GR C, AIR CLAMP

$.9510 D$ DURSTINO 8TRBNOTH UP TO 45 PSI. PBRIINS CA OR C, $\triangle I R$ CLAMP 1.40 $10 D$ BUR BTINO STRBNOTB UP TO 45 PSI, PBRIINS CA OR C, $\triangle I R$ CLAMP $1.0210 \mathrm{D}$ BURATINO BTRBNOTH UP TO 45 PaI, PBREINS CA OR C, AIR CLAMP $1.0510 D$ BURETINO STRBNOTR UP TO 45 P8I. PBREINS CA OR C, AIR CLANP $1.1610 D$ BURETIMO STRBNOTH UP TO 45 PSI. PBREINS CA OR C. $A I R$ CLAMP

1.31 10 D BURETINO BTRBNOTB UP TO 45 PSI, PBRIINS CA OR C, AIR CLAMP

1.00

VITB OAMM - SS DEORBER 
BURSTING STRENGTH, MOOEL $\mathrm{C}-\mathrm{A}$

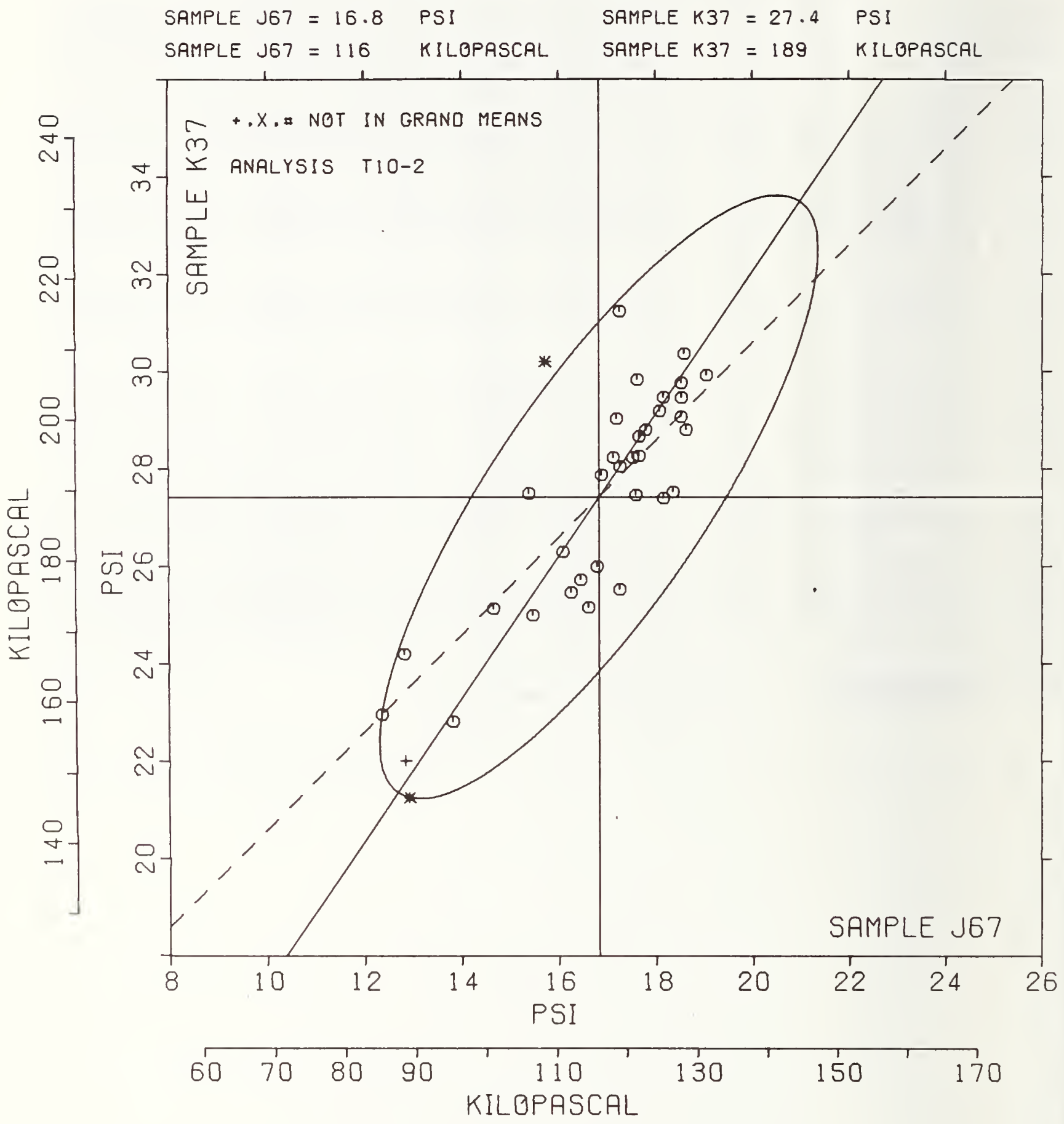


TAPPI 9TANDARD T\$03 08-76. BURBTING 8TRBNGTE OP PAPBR PBREINB MODBL C OP C-A

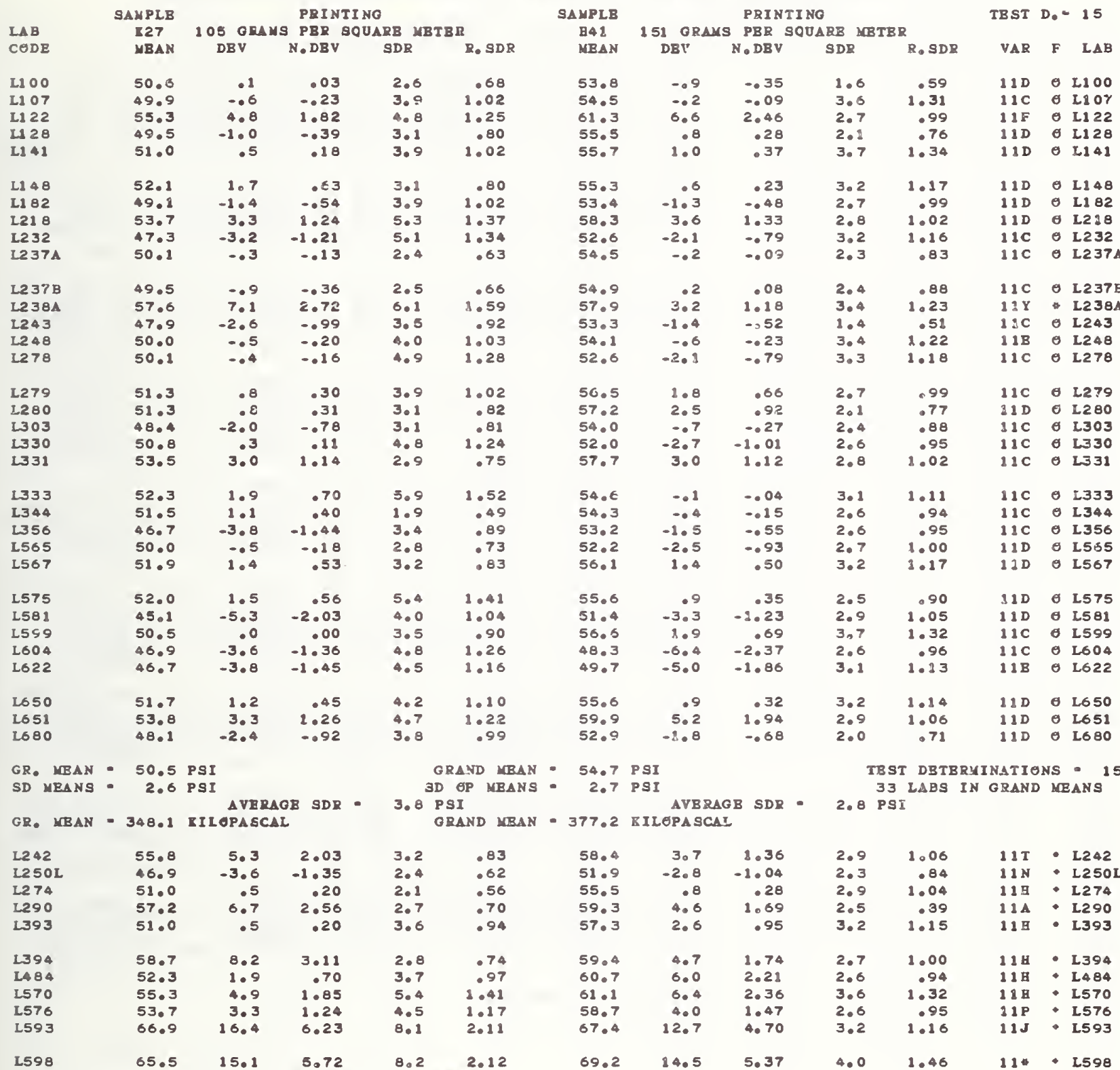

TOTA NUMBBP OP $15.125 .72 \quad 8.2$

Best values: $\mathrm{K} 2750 \pm 4 \mathrm{ps} 1$

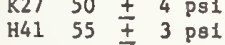


TAPPI STANDARD T403 69-76, BURGTING STRBNGTB OF PAPBR - PBRINS MODEL C OR C-A

\begin{tabular}{|c|c|c|c|c|c|c|c|c|c|c|c|c|c|}
\hline LAB & & MBA & & CEORDI & $\mathrm{N} \triangle \mathrm{TBS}$ & AVG & & & & & & & \\
\hline $\mathrm{CODB}$ & $\mathbf{F}$ & R27 & H41 & MAJOB & MINOR & R. SDR & VAR & PROPBRT & IY---TBST & I N 8 & BTRUMBN & NT- - CENDITIEN & \\
\hline L581 & $\theta$ & $45 \cdot 1$ & 51.4 & $-6 \cdot 1$ & 1.5 & 1.05 & $11 \mathrm{D}$ & BURSTING & STRBNOTB & 40 & -100 & PSI. PBRIINS & CA, AIR CLAMP \\
\hline L622 & $\theta$ & 46.7 & 49.7 & $-6 \cdot 3$ & -7 & 1.15 & $11 \mathrm{~B}$ & BURETINO & STRBNOTB & 40 & -100 & PSI. PEREINS & C, MANUAL CLAYP \\
\hline L3 56 & $\theta$ & 46.7 & $53 \cdot 2$ & -3.7 & 1.7 & .92 & $11 \mathrm{C}$ & BURSTING & STRBNGTB & 40 & -100 & PSI, PBREINS & C. MANUAL CLAMP \\
\hline L6O4 & $\theta$ & 46.9 & 48.3 & -7.1 & -1.9 & 1.11 & $11 \mathrm{C}$ & BURSTING & STRBNOTB & 40 & -100 & PSI. PBREI NS & C, MANDAL CLAMP \\
\hline L250L & $\bullet$ & 46.9 & 51.9 & $-4 \cdot 5$ & .6 & .73 & $12 \mathrm{~N}$ & BURSTING & STRBNGTB & 40 & -100 & PSI, LBEMARGY, & MAN, CLAKP, 2OC,65 XRB \\
\hline L232 & $\theta$ & $47 \cdot 3$ & 52.6 & -3.8 & .8 & 1.25 & $11 \mathrm{C}$ & BURETING & STRBNOTB & 40 & -100 & PSI, PBRIINS & C, MANUAL CLAMP \\
\hline L243 & $\boldsymbol{\theta}$ & 47.9 & 53.3 & $-2 \cdot 8$ & .9 & .71 & $11 \mathrm{C}$ & BURgTING & STRB NGTB & 40 & -100 & PQI, PBRIINS & C. MNUAL CLAMP \\
\hline L680 & $\theta$ & 48.1 & 52.9 & -3.0 & .5 & .85 & $11 \mathrm{D}$ & BURSTINO & बTRBNGTB & 40 & -100 & PSI, PBREINS & CA, AIR CLAMP \\
\hline 2303 & $\theta$ & 48.4 & 54.0 & $-2 \cdot 0$ & 1.0 & .85 & $11 \mathrm{C}$ & BURETINO & GTRENOTB & 40 & -100 & P8I, PBREINS & C. MANUAL CLAMP \\
\hline LI 82 & $\theta$ & 49.1 & 53.4 & $-1 \cdot 9$ & .1 & 1.00 & $11 \mathrm{D}$ & BURSTING & STRBNGTE & 40 & -100 & PQI, PBREINS & CA, $\triangle I R$ CLAMP \\
\hline L128 & $\theta$ & 49.5 & 55.5 & $\bullet 2$ & $1 \cdot 3$ & .78 & $11 \mathrm{D}$ & BURSTING & STRBNGTB & 40 & -100 & PSI, PBRIINS & CA, AIR CLAMP \\
\hline L237B & $\boldsymbol{\theta}$ & 49.5 & $54 \cdot 9$ &. .5 & - 8 & $\bullet 77$ & $11 \mathrm{C}$ & BURSTING & STRBNGTB & 40 & -100 & PSI, PBDINB & C. MANUAL CLAMP \\
\hline 2807 & $\theta$ & 49.9 & 54.5 & -.6 & .3 & 1.16 & $12 \mathrm{C}$ & BURSTING & STRBNGTB & 40 & -100 & PSI, PBREINS & C. YANUAL CLAMP \\
\hline $\mathrm{L} 248$ & $\theta$ & 50.0 & 54.1 & -8 & $=0$ & 1.12 & $11 \mathrm{~B}$ & BURSTINO & STRBNGTB & 40 & -100 & PQI, PBRIINS & C. MANOAL CLAMP \\
\hline 2565 & $\theta$ & 50.0 & $52 \cdot 2$ & $-2 \cdot 1$ & $-1 \cdot 4$ & .86 & $11 \mathrm{D}$ & BURATING & GTRBNGTB & 40 & -100 & PSI, PBRIINS & CA. AIR CEAMP \\
\hline L278 & $\theta$ & 50.1 & 52.6 & -1.8 & $-1 \cdot 2$ & 1.23 & $11 \mathrm{C}$ & BURSTING & STRBNGT B & 40 & -100 & PSI, PSRIINS & C. MANULL CLAMP \\
\hline 22374 & $\theta$ & 50.1 & $54 \cdot 5$ &. .4 & $\bullet 1$ & .73 & $11 \mathrm{C}$ & BURGTING & STRBNOTB & 40 & -100 & PSI, PBRIINS & C. MANUAL CLAMP \\
\hline L599 & $\theta$ & 50.5 & 56.6 & 1.4 & $1 \cdot 3$ & $1 \cdot 11$ & $11 \mathrm{C}$ & BUR IT ING & GTRBNCTB & 40 & -100 & PQI, PBRIINS & C. MANUAL CLAMP \\
\hline L100 & $\theta$ & 50.6 & $53 \cdot 8$ & -.6 & .7 & .64 & $11 \mathrm{D}$ & BURGTING & STRBNGTE & 40 & -100 & PQI, PBRIINS & CA, IIR CLAMP \\
\hline 2330 & $\theta$ & 50.8 & $52 \cdot 0$ & -1.8 & -2.1 & 1.09 & $11 \mathrm{C}$ & BURgTING & STRBNGTH & 40 & -100 & PSI, PERIINS & C. MANUAL CLAMP \\
\hline 2148 & $\theta$ & 52.0 & 5.7 & 1.1 & $\cdot 3$ & 1.18 & $12 \mathrm{D}$ & BURATING & STRBNGTB & 40 & -100 & PSI, PBRIINS & CA, AIR CLAMP \\
\hline 1393 & $\bullet$ & $51 \cdot 0$ & $57 \cdot 3$ & $2 \cdot 2$ & $1 \cdot 4$ & 1.04 & $11 \mathrm{~B}$ & BURGT ING & STRBNGTH & 40 & -100 & PSI, PBREINS & AB, BYDRA UL IC CLAMP \\
\hline 1274 & $\bullet$ & 51.0 & 55.5 & .9 & $\cdot 2$ & .80 & $11 \mathrm{~B}$ & BURSTING & STRBNGTB & 40 & -100 & PQI, PBRIINS & $\triangle B$, BYDR OL IC CLAMP \\
\hline 1279 & $\theta$ & $51 \cdot 3$ & 56.5 & $1 \cdot 8$ & .7 & 1.00 & $11 \mathrm{C}$ & BUR ST ING & STRBNGTE & 40 & -100 & PSI, PBRIINS & C. MANUAL CLAMP \\
\hline 2280 & $\theta$ & $52 \cdot 3$ & 57.2 & $2 \cdot 4$ & $1 \cdot 1$ & .79 & 11D & BURSTING & STRBNGTH & 40 & -100 & PSI, PBRIINS & CA, AIR CLAMP \\
\hline $\mathbf{L 3 4 4}$ & $\theta$ & 51.5 & $54 \cdot 3$ & .4 & $-1 \cdot 0$ & .72 & $11 \mathrm{C}$ & BURST ING & STRBNGTH & 40 & -100 & PSI, PBRIINS & C. MANUAL CLAMP \\
\hline L650 & $\boldsymbol{\theta}$ & $52 \cdot 7$ & 55.6 & $1 \cdot 4$ & $\bullet 3$ & 1.12 & $11 \mathrm{D}$ & BURgTING & STRBNGTB & 40 & -100 & PSI, PBRIINS & CA, AIR CLAMP \\
\hline L567 & $\theta$ & 51.9 & 56.1 & 1.9 & -.0 & 1.00 & $11 \mathrm{D}$ & BURGT ING & STRBNGTB & 40 & -100 & PSI, PBRIINS & CA. AIR CLAMP \\
\hline L575 & $\theta$ & 52.0 & 55.6 & $1 \cdot 7$ & $-\bullet 4$ & 1.16 & $11 \mathrm{D}$ & BURGTING & STRBNGTB & 40 & -100 & PSI, PBRIINS & CA. $\triangle I R$ CLAMP \\
\hline L\$48 & $\theta$ & $52 \cdot 1$ & $55 \cdot 3$ & 1.6 & -68 & .99 & 110 & BURSTING & STRBNGTH & 40 & -100 & PQI, PBRIINS & CA, AIR CLAMP \\
\hline 2484 & $\bullet$ & $52 \cdot 3$ & 60.7 & 5.6 & $2 \cdot 8$ & .95 & $11 \mathrm{H}$ & BURST ING & STRBNGTE & 40 & -100 & PQI, PBRIINS & AB, BYDRAULIC CLAKP \\
\hline L3 33 & $\theta$ & $52 \cdot 3$ & 54.6 & $1 \cdot 2$ & $-2 \cdot 4$ & 1.32 & $11 \mathrm{C}$ & BURGTINO & OTRBNGTB & 40 & -100 & PSI, PBRIINS & C. MANUAL CLAMP \\
\hline L332 & $\theta$ & $53 \cdot 5$ & 57.7 & $4 \cdot 3$ & -.0 & .88 & $11 \mathrm{C}$ & BORATING & ATRBNGTB & 40 & -100 & PQI, PBRIINS & C, MANUAL CLAMP \\
\hline 2576 & $\bullet$ & $53 \cdot 7$ & 58.7 & 5.1 & .4 & 1.06 & $11 \mathrm{P}$ & BURATING & STRBNGTE & 40 & -100 & PSI, PBRIINS & LC, MANUAL CLAMP \\
\hline L21 8 & $\theta$ & 53.7 & $58 \cdot 3$ & 4.8 & .2 & 1.20 & $11 \mathrm{D}$ & BURATING & STRBNCTE & 40 & -100 & PQI, PBRIINS & CA, $\triangle I R$ CLAMP \\
\hline L651 & $\theta$ & 53.8 & 59.9 & 6.1 & $1 \cdot 3$ & 1.14 & $11 \mathrm{D}$ & BURATING & ATRBNGTE & 40 & -100 & PQI, PBRIINS & $\triangle I R$ CLAMP \\
\hline L122 & $\theta$ & 55.3 & $61 \cdot 3$ & 8.1 & $1 \cdot 2$ & 1.12 & $11 \mathrm{P}$ & BURAIINO & GTRBNGTH & 40 & -100 & PQI, PBREINSC & C, H. CLAMP, TRANA DUCER \\
\hline $\mathbf{L} 570$ & - & $55 \cdot 3$ & $61 \cdot 1$ & $7 \cdot 9$ & - 9 & 1.36 & $11 \mathrm{~B}$ & BURETINO & ВTRB NGTH & 40 & -100 & PQI. PBRIING & $\triangle B$, HYDRAULIC CLAMP \\
\hline $\mathrm{L} 242$ & $\bullet$ & $55 \cdot 8$ & $58 \cdot 4$ & 6.4 & $-1 \cdot 3$ & - 94 & $11 \mathrm{~T}$ & BURgTINO & GTRBNGTE & 40 & -100 & $P Q I, L+\pi, M A N D$ & OAL CLAMP \\
\hline L290 & - & $57 \cdot 2$ & 59.3 & $7 \cdot 9$ & $-1 \cdot 7$ & .79 & 114 & BURSTINO & STRBNGTB & 40 & -100 & PQI, PERIINS & A. MANUAL CLAMP \\
\hline L238A & - & 57.6 & 57.9 & $7 \cdot 3$ & -2.9 & 1.41 & $11 Y$ & BURETING & ATRENGTE & 40 & -100 & PSI, PBRIINS & CA, AIR CLAMP \\
\hline L394 & $\bullet$ & 58.7 & 59.4 & 9.1 & -2.6 & - 87 & $11 \mathrm{~B}$ & BURSTING & ATRBNGTB & 40 & -100 & PEI. PBRIING & AB, BYDRAULIC CLAMP \\
\hline L598 & $\bullet$ & $65 \cdot 5$ & 69.2 & 20.9 & -.7 & 1.79 & $11 \cdot$ & BUR ITING & STRBNOTE & 40 & -100 & PQI, MBSEMER, & MNUAL CLAKP \\
\hline L5\$3 & - & 66.9 & 67.4 & 20.5 & -2.9 & 1.64 & $11 \mathrm{~J}$ & BURSTING & STRBNGTH & 40 & -100 & PQI, PBRIINS & J UMBE, HAND DRIVBN \\
\hline & & $\begin{array}{l}50.5 \\
95 \% \mathrm{~B}\end{array}$ & $\begin{array}{c}54 \cdot 7 \\
\text { L I P SB: }\end{array}$ & 9.4 & 3.0 & $\begin{array}{l}1.00 \\
\text { ITH }\end{array}$ & $O \Delta M I$ & $M \Delta-45$ & PBB 9 & & & & \\
\hline
\end{tabular}


BURSTING STRENGTH, HIGH RANGE

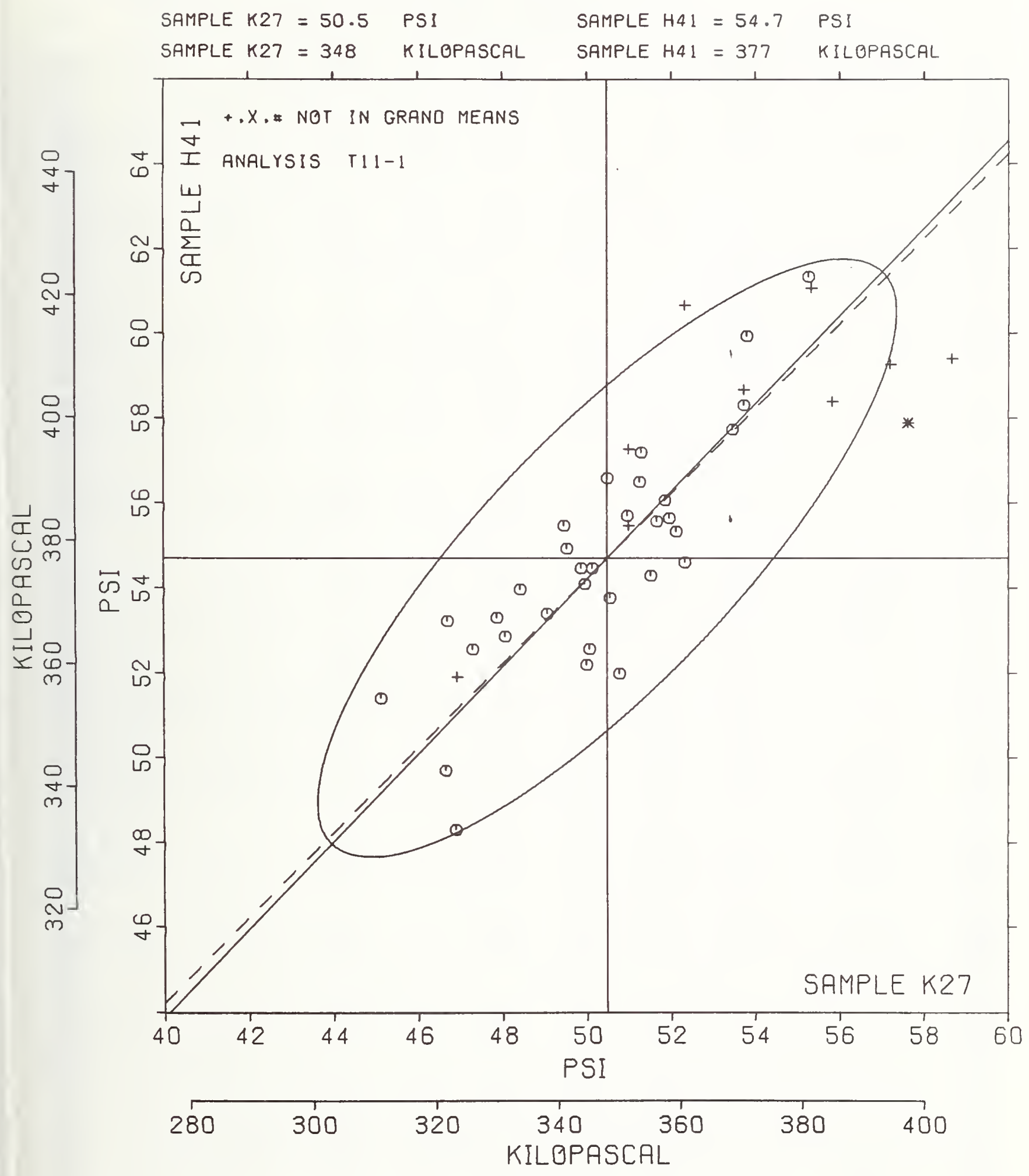


TAPPI STANDARD 1414 IS-65, ANY MAKB BLIGNDGRF FITB DEEP CUTGUT IS BTANDARD FOR IBIS ANALYSIS

\begin{tabular}{|c|c|c|c|c|c|c|c|c|c|c|c|c|c|}
\hline \multirow{3}{*}{$\begin{array}{l}\text { LAB } \\
\text { CबDB }\end{array}$} & \multirow{3}{*}{$\begin{array}{c}\text { SAMPLB } \\
\text { K25 } \\
\text { MBAN }\end{array}$} & \multicolumn{4}{|c|}{ PRINTINO } & \multirow{3}{*}{$\begin{array}{c}\text { SAMPLB } \\
\text { B85 } \\
\text { MBAN }\end{array}$} & \multicolumn{4}{|c|}{ BOND } & \multirow{2}{*}{ TBST } & \multirow{2}{*}{$\mathrm{D}_{0}=$} & \multirow{2}{*}{ - 15} \\
\hline & & 75 GEAI & IS PBR 8 & $A R B$ MB & & & 79 GRA & M 8 PBR \& & ARB IA & & & & \\
\hline & & DBV & $N \cdot D B V$ & SDR & $R_{\bullet} \mathbf{S D R}$ & & DBV & $\mathrm{N} \cdot \mathrm{DB} \mathbf{V}$ & SDR & R. SDR & $\mathbf{V A R}$ & $\mathbf{F}$ & $\mathbf{L A B}$ \\
\hline L100 & $41 \cdot 3$ & $-1 \cdot 1$ & -.051 & .6 & .40 & 39.7 & -.3 & .15 & 1.0 & .81 & $15 \mathrm{M}$ & $\sigma$ & 2100 \\
\hline L105 & 42.7 & $\cdot 3$ & .12 & $2 \cdot 6$ & 1.74 & 40.5 & .6 & .26 & $1 \cdot 2$ & .94 & $15 \mathrm{~T}$ & $\theta$ & L105 \\
\hline L8 07 & 44.0 & 1.6 & .73 & 1.3 & .88 & 41.7 & $1 \cdot 8$ & - 81 & .7 & .56 & $15 \mathrm{~T}$ & $\boldsymbol{\theta}$ & L107 \\
\hline L121 & 35.6 & -6.8 & -3.11 & $2 \cdot 6$ & 1.78 & 35.6 & -4.4 & -2.02 & $1 \cdot 5$ & 1.23 & $15 \mathrm{~T}$ & $\bullet$ & L1 21 \\
\hline L1 22 & $43 \cdot 8$ & $1 \cdot 4$ & .65 & 1.4 & - 56 & $43 \cdot 3$ & $3 \cdot 3$ & 1.54 & $1 \cdot 2$ & .99 & $15 \mathrm{C}$ & $\theta$ & 2122 \\
\hline 1124 & 42.1 & -3 &. .12 & 2.1 & $1 \cdot 39$ & 40.5 & .6 & .26 & $2 \cdot 3$ & 2.85 & $15 \mathrm{~T}$ & $\theta$ & I1 24 \\
\hline L126 & $42 \cdot 9$ & .5 & .21 & 1.7 & 1.14 & $41 \cdot 8$ & $1 \cdot 8$ & . 85 & 1.7 & 1.35 & $15 T$ & $\theta$ & I1 26 \\
\hline L\$28 & 42.4 & .0 & -.00 & $1 \cdot 2$ & .84 & 40.7 & .7 & .32 & .7 & .57 & $15 \mathrm{~T}$ & $\theta$ & L128 \\
\hline L832 & 47.7 & $5 \cdot 3$ & 2.40 & 1.0 & .66 & 45.3 & 5.3 & 2.45 & .7 & .56 & $15 \mathrm{~A}$ & - & L1 31 \\
\hline 1834 & 40.9 & -1.5 &. .70 & $\cdot 8$ & .56 & 39.7 & -.2 & -.11 & .9 & .70 & $15 \mathrm{C}$ & $\theta$ & I1 34 \\
\hline L139 & 43.9 & 1.5 & .70 & 1.0 & .70 & $41 \cdot 6$ & 1.8 & .85 & .7 & .54 & $15 \mathrm{~T}$ & $\theta$ & L1 39 \\
\hline L141 & 42.3 & -01 & -.06 & 1.5 & 1.00 & 39.5 & -.5 & $\because 23$ & .9 & .73 & $15 \mathrm{~T}$ & $\theta$ & I141 \\
\hline L 143 & 35.4 & -7.0 & -3.20 & 1.6 & 1.19 & 29.9 & -10.1 & -4.68 & 4.1 & 3.26 & $15 \mathrm{~T}$ & $*$ & I143 \\
\hline L1 45 & 8.5 & -33.9 & $-15 \cdot 46$ & $\bullet 8$ & .56 & $7 \cdot 8$ & $-32 \cdot 2$ & -14.88 & .4 & .33 & $15 x$ & $\bullet$ & I145 \\
\hline 2148 & 40.5 & -1.9 & -85 & $\cdot \theta$ & .56 & 39.5 & -.4 & -.20 & .5 & .41 & $15 \mathrm{~T}$ & $\sigma$ & 2148 \\
\hline II 50 & 53.0 & 10.6 & 4.83 & $1 \cdot 1$ & .72 & 50.3 & 10.4 & 4.79 & .8 & .65 & $15 \mathrm{~T}$ & 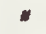 & L1 50 \\
\hline L1 51 & 53.1 & 80.7 & 4.86 & 2.9 & 1.98 & 47.7 & 7.8 & 3.59 & $1 \cdot 2$ & .92 & $15 \mathrm{C}$ & $*$ & I1 51 \\
\hline L153 & 42.3 &. .1 &. .03 & 1.4 & .98 & 39.1 & -08 & -.39 & .7 & .59 & $15 \mathrm{C}$ & $\sigma$ & 2153 \\
\hline L157 & $41 \cdot 8$ & -.6 & $-.2 \theta$ & 2.1 & 1.40 & 39.3 & .06 & -.30 & .8 & .65 & $15 \mathrm{~T}$ & 6 & L1 57 \\
\hline 2158 & $41 \cdot 6$ & -08 & -.37 & 1.7 & 1.16 & 40.1 & $\cdot 2$ & .07 & 3.6 & 2.84 & $15 R$ & $\sigma$ & 2158 \\
\hline L162 & 43.3 & .9 & .42 & 1.0 & $\bullet \in 6$ & 40.5 & .6 & .26 & 1.4 & 1.12 & $15 \mathrm{~T}$ & $\theta$ & 1162 \\
\hline L1 63 & 44.2 & 1.8 & .82 & 1.8 & 1.23 & 39.4 & -.6 &. .26 & $1 \cdot 2$ & .99 & $15 \mathrm{~T}$ & $\theta$ & 1163 \\
\hline 2166 & 42.4 & -00 &. .00 & $1 \cdot 2$ & .80 & 39.5 & $\bullet 5$ & -.23 & $1 \cdot 1$ & .84 & $15 \mathrm{~T}$ & $\theta$ & 2166 \\
\hline L167 & 41.9 & $\because .5$ & -.25 & 1.8 & 1.19 & $39 \cdot 3$ & -.66 & -.30 & 1.2 & .98 & $15 \mathrm{C}$ & $\sigma$ & 2167 \\
\hline$L 273 B$ & 44.5 & $2 \cdot 1$ & .97 & 1.4 & .95 & $41 \cdot 3$ & $1 \cdot 4$ & .63 & 1.0 & .77 & $15 \mathrm{~T}$ & $\theta$ & 21738 \\
\hline L1 $82 A$ & 43.3 & .9 & .39 & $2 \cdot 3$ & 1.52 & 38.3 & $-1 \cdot 7$ & .79 & $1 \cdot 3$ & 1.06 & 154 & $\boldsymbol{\theta}$ & II $82 A$ \\
\hline L1 82T & 46.9 & 4.5 & 2.07 & 1.4 & - 97 & $43 \cdot 8$ & $3 \cdot 8$ & 1.77 & .9 & .68 & $15 \mathrm{~T}$ & $\theta$ & L1 $82 \mathrm{~T}$ \\
\hline L1 83 & 41.8 & -06 & -.28 & .9 & .58 & 37.5 & $-2 \cdot 5$ & -1.16 & .8 & .66 & $15 T$ & $\theta$ & L1 83 \\
\hline L1 85 & 42.8 & .4 & .18 & 1.7 & 1.18 & 41.3 & 1.4 & .63 & .8 & .65 & $15 \mathrm{~T}$ & $\theta$ & I1 85 \\
\hline 1289 & 43.0 & .6 & .27 & $1 \cdot 5$ & 1.02 & $41 \cdot 8$ & 1.8 & .85 & .9 & .75 & $15 \mathrm{~T}$ & $\sigma$ & L189 \\
\hline $\mathrm{L1} 90 \mathrm{C}$ & 43.1 & .7 & .33 & 1.1 & .76 & 39.5 & -.4 &. .20 & .7 & .59 & $15 T$ & $\sigma$ & $2190 \mathrm{C}$ \\
\hline $\mathrm{LI} 90 \mathrm{R}$ & 40.9 & $-1 \cdot 5$ & -.70 & - 8 & .56 & 37.7 & $-2 \cdot 3$ & -1.07 & .6 & .49 & $15 \mathrm{C}$ & $\sigma$ & L190R \\
\hline L192 & 36.1 & $-6 \cdot 3$ & -2.86 & $1 \cdot 8$ & 1.19 & 34.4 & -5.6 & $-2 \cdot 58$ & 1.1 & .89 & $15 \mathrm{~T}$ & - & L191 \\
\hline L194 & 45.1 & 2.7 & 1.24 & 1.2 & .79 & 43.6 & 3.6 & 1.66 & 1.2 & .94 & $15 \mathrm{~T}$ & $\sigma$ & 1194 \\
\hline L195 & 44.1 & 1. 7 & .79 & $1 \cdot 8$ & 1.19 & $39 \cdot 7$ & -.2 & -011 & $1 \cdot 5$ & 1.18 & $15 \mathrm{C}$ & $\boldsymbol{\sigma}$ & L195 \\
\hline 2206 & 42.9 & .5 & .21 & 1.1 & .76 & 39.5 & -.5 & -.23 & 1.0 & .79 & $15 R$ & $\boldsymbol{\theta}$ & L206 \\
\hline 2207 & 51.2 & e. 7 & 3.99 & $2 \cdot 3$ & 1.53 & 49.5 & 9.5 & 4.40 & 1.5 & 1.17 & $15 R$ & $*$ & L207 \\
\hline 2211 & $42 \cdot 3$ & -.1 & -.03 & 1.3 & .87 & 41.4 & 1.4 & .66 & $1 \cdot 7$ & $1 \cdot 33$ & $15 R$ & 6 & L211 \\
\hline Lâ1 2 & 43.9 & 1.5 & .67 & 1.6 & 1.11 & 40.7 & $\bullet 8$ & $\cdot 35$ & $1 \cdot 8$ & 1.39 & $15 \mathrm{~T}$ & $\theta$ & 1212 \\
\hline 1213 & 44.3 & 1.5 & .85 & 1.3 & .86 & 42.0 & 2.0 & .94 & $1 \cdot 3$ & 1.04 & $15 \mathrm{~T}$ & $\theta$ & L213 \\
\hline 1217 & 43.2 & .8 & .36 & 1.2 & .80 & 41.5 & 1.5 & .70 & .7 & .53 & $15 T$ & $\sigma$ & $L 217$ \\
\hline 1219 & 43.2 & - 8 & .36 & $2 \cdot 1$ & 1.43 & $41 \cdot 3$ & 1.4 & .63 & 1.0 & .77 & $15 \mathrm{~L}$ & $\boldsymbol{\theta}$ & 1219 \\
\hline L223 & 43.8 & 1.4 & .64 & 1.0 & .70 & 40.3 & .3 & .16 & .9 & .71 & $15 R$ & $\sigma$ & L223 \\
\hline$\lcm{2225}$ & $43 \cdot 3$ & .9 & .39 & 1.0 & .70 & 43.4 & 3.4 & 1.59 & . 8 & .56 & $15 \mathrm{~T}$ & $\sigma$ & L2 25 \\
\hline L226B & $43 \cdot 3$ & .9 & .42 & $2 \cdot 2$ & 1.50 & 40.0 & .0 & .01 & $1 \cdot 5$ & 1.20 & $15 T$ & $\theta$ & L226 B \\
\hline $1226 C$ & 39.2 & $-3 \cdot 2$ & $-1 \cdot 45$ & 1.4 & .91 & 36.2 & -3.7 & -1.73 & .8 & .64 & $15 \mathrm{~T}$ & $\theta$ & $L 226 C$ \\
\hline L228 & $46 \cdot 3$ & 3.9 & 1.76 & 2.0 & 1.34 & 40.7 & .7 & - 32 & 2.0 & 1.55 & $15 \mathrm{~T}$ & - & L228 \\
\hline 1232 & 42.0 & -.4 & -.19 & 1.7 & 1.14 & 39.6 & -.4 & -17 & 1.9 & 1.49 & $15 \mathrm{~T}$ & $\boldsymbol{\theta}$ & L232 \\
\hline 1236 & 45.7 & $3 \cdot 3$ & 1.52 & $1 \cdot 5$ & 1.04 & $42 \cdot 7$ & 2.7 & 1.25 & 1.5 & 1.19 & $15 \mathrm{~T}$ & $\sigma$ & 1236 \\
\hline $1237 A$ & 40.7 & $-1 \cdot 7$ & .076 & $1 \cdot 3$ & .90 & 37.8 & $-2 \cdot 2$ & -1.00 & $1 \cdot 1$ & .86 & $15 \mathrm{~T}$ & $\sigma$ & L237A \\
\hline $1237 \mathrm{~B}$ & 43.0 & .6 & .27 & 1.0 & .68 & 40.6 & .6 & .29 & .9 & .72 & $15 \mathrm{~T}$ & $\theta$ & L237B \\
\hline L238A & 40.7 & $-1 \cdot 7$ & -.76 & 1.4 & .94 & 39.3 & -.7 & -.33 & 1.2 & .97 & $15 \mathrm{~T}$ & $\sigma$ & L2 384 \\
\hline 2241 & 44.7 & $2 \cdot 3$ & 1.06 & 1.1 & .74 & 43.1 & 3.1 & 1.43 & .8 & .63 & $15 \mathrm{~T}$ & $\theta$ & L241 \\
\hline 1243 & $42 \cdot 8$ & .4 & .18 & 1.2 & .82 & 39.6 & -04 & -11 & .7 & .58 & $15 \mathrm{~T}$ & $\sigma$ & L243 \\
\hline 2244 & 45.3 & 2.9 & $1 \cdot 31$ & 1.3 & .90 & $43 \cdot 8$ & 3.8 & 1.77 & $1 \cdot 5$ & 1.21 & $15 \mathrm{C}$ & $\boldsymbol{\sigma}$ & 1244 \\
\hline 1248 & $42 \cdot 6$ & .2 & .10 & 1.9 & $1 \cdot 2 \theta$ & 40.3 & .4 & .16 & .5 & .42 & $15 J$ & $\sigma$ & 1248 \\
\hline 2249 & 40.4 & -2.0 & -.92 & 1.4 & .95 & 37.6 & $-2 \cdot 4$ & -1.10 & $1 \cdot 1$ & .84 & $15 \mathrm{~T}$ & $\sigma$ & 1249 \\
\hline 1254 & 44.9 & 2.5 & 1.15 & 1.5 & 1.00 & 40.6 & .8 & $\cdot 38$ & $1 \cdot 3$ & 1.00 & $15 \mathrm{~T}$ & $\theta$ & L254 \\
\hline L255 & 42.3 & -.1 & -.006 & .5 & .31 & 38.6 & -1.4 & -63 & .5 & .40 & $15 \mathrm{~T}$ & $\sigma$ & L255 \\
\hline $1257 A$ & $43 \cdot 5$ & 1.1 & .48 & 1.2 & . 80 & 41.6 & 1.6 & .75 & - 8 & .66 & $15 \mathrm{C}$ & $\theta$ & $2257 A$ \\
\hline$L 257 \mathrm{~B}$ & 43.5 & 1.1 & .48 & 1.4 & .95 & 42.0 & 2.0 & .94 & $1 \cdot 3$ & 1.04 & $15 \mathrm{C}$ & $\boldsymbol{\sigma}$ & L257B \\
\hline $1257 \mathrm{C}$ & 43.1 & .7 & .30 & 1.7 & 1.13 & 41.9 & 1.9 & .88 & 1.2 & .94 & $15 \mathrm{C}$ & $\boldsymbol{\sigma}$ & L257C \\
\hline L259 & 42.3 & -1 & -.03 & 1.8 & 1.19 & 40.9 & 1.0 & .44 & $1 \cdot 2$ & .92 & $15 T$ & $\sigma$ & L259 \\
\hline L261 & 41.5 & -.9 & -.40 & 1.3 & .88 & 37.9 & -2.0 & .94 & 1.0 & .76 & $15 \mathrm{~T}$ & $\theta$ & L261 \\
\hline 1262 & 42.1 & -.3 & -.15 & 1.1 & .74 & 40.0 & .0 & .01 & $1 \cdot 1$ & .90 & $15 \mathrm{~T}$ & $\sigma$ & $L 262$ \\
\hline
\end{tabular}


ANAYSIR T1S-1 TABLB

TAPPI STANDARD T414 TB-65, ANY MEB BLMBNDGRF IITB DBBP CUTOUT IB STANDARD POR TBIS ANALYSIS

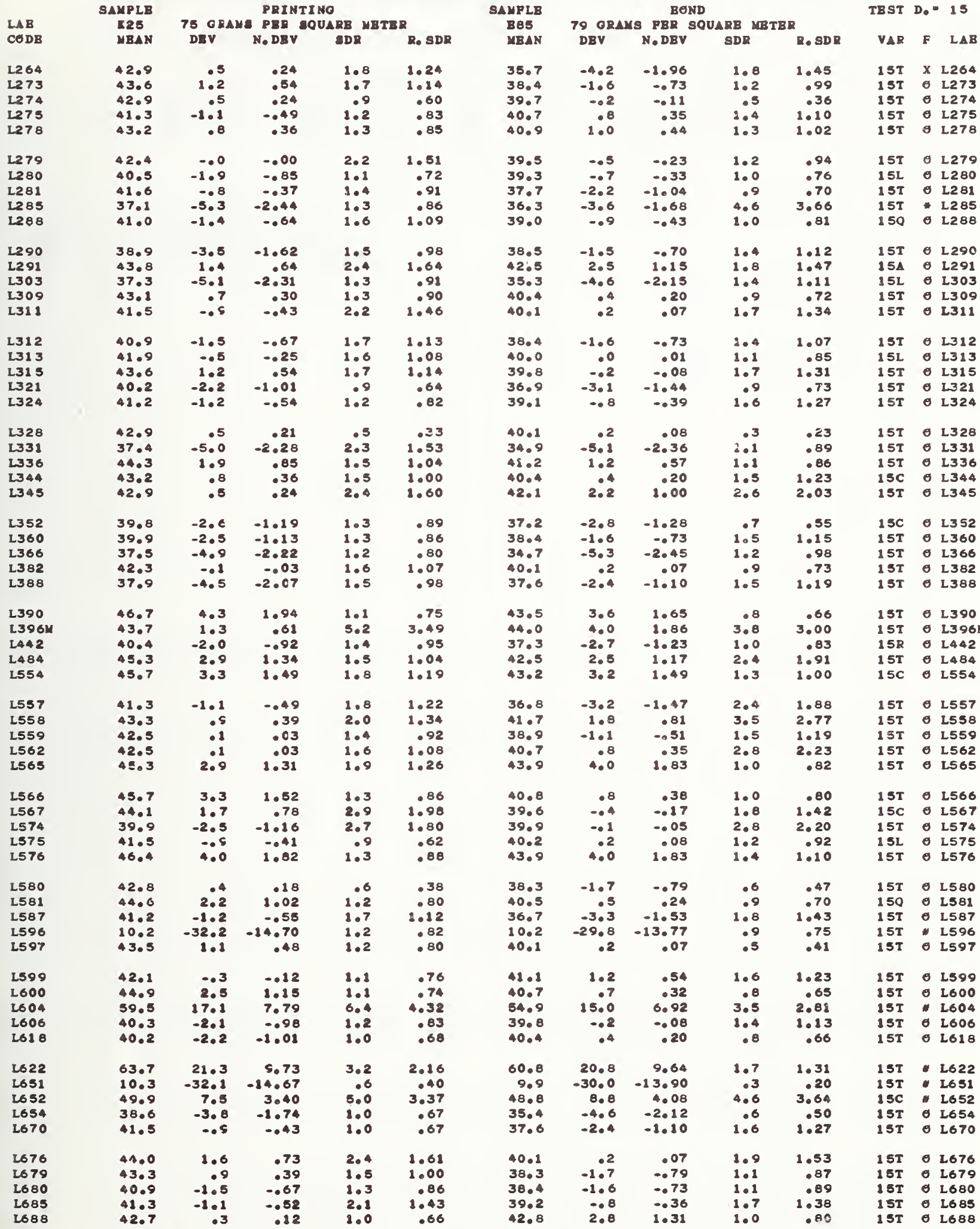

OR. MBAN - 42.4 GRAMS GRAND MBAN - 40,0 GRAMS

SD MRANS - 202 ORAM

SD MRAS 40.0 GRM

TBST DETBRMINATIONS - 15

110 LABS IN GRAND NEANS

$\triangle V E R A O B$ SDR - 1.5 ORAMS

AVBRAGB SDR - 1.3 GRAMS

GR. MEAN - 415.9 UILLIYBVTON

GRAND MBAN - 392.0 UILLINBWTON 


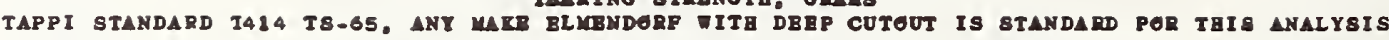

\begin{tabular}{|c|c|c|c|c|c|}
\hline \multirow{3}{*}{$\begin{array}{l}\text { LAB } \\
C E D E\end{array}$} & \multicolumn{2}{|l|}{ SAMPLB } & \multicolumn{3}{|c|}{ PRINTINO } \\
\hline & R25 & 75 GLAMS & PBR \& & $\triangle R B$ & \\
\hline & MBAK & DEV & N.DBV & SDR & $\mathbf{R} \cdot \mathbf{8}$ \\
\hline 1230 & 39.1 & $-3 \cdot 3$ & -1.49 & 1.4 & .94 \\
\hline $\mathrm{L} 242$ & 43.6 & 1.2 & .54 & 1.8 & 1.22 \\
\hline L250L & 45.4 & 3.0 & 1.38 & 1.2 & .81 \\
\hline 1299 & 45.4 & 3.0 & $1 \cdot 37$ & 1.4 & .91 \\
\hline L680 & 40.5 & -1.5 & .085 & 1.9 & 1.30 \\
\hline 1684 & 33.7 & -8.7 & -3.96 & 1.8 & 1.10 \\
\hline \multicolumn{6}{|c|}{ TETAL NUMBER GF LABEDATEDIBE LBPCRTINO - 136} \\
\hline Best values: & $\begin{array}{ll}\text { K25 } & 43 \\
\text { E85 } & 40\end{array}$ & \pm 3 grams & & & \\
\hline
\end{tabular}

The following laboratorles were onitted from the grand means because of extreme test results: 143 , $150,151,207,604,622,652$.

Data from the following laboratories appear to be off by a multiplicative factor: 145, 596, 651 .

Data from the following laboratories appeared to be off by a multiplicative factor: $230,299$. Code $15 \mathrm{~V}$ was assigned temporarily to put in a factor of 2 .

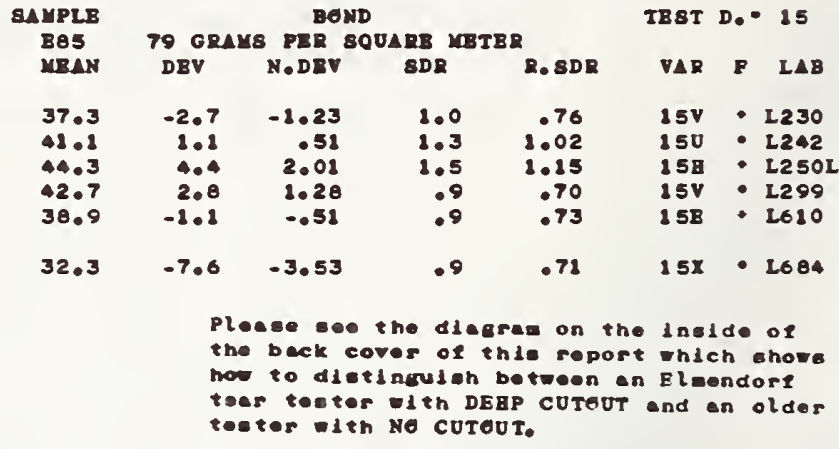


IAPPI STANDARD 1414 TS-65, ANY MAKB BLNENDERP TITE DBEP CUIEUT IS STANDARD FER TBIS ANALYSIS

\begin{tabular}{|c|c|c|c|c|c|}
\hline \multicolumn{2}{|l|}{ LAB } & \multicolumn{2}{|c|}{ MBANS } & \multicolumn{2}{|c|}{ CEGRDINATBS } \\
\hline$C E D B$ & $\mathbf{F}$ & B25 & E85 & MAJOR & MINO \\
\hline L145 & $\bullet$ & 8.5 & 7.8 & -46.7 & \\
\hline L596 & 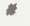 & 10.2 & 10.2 & $-43,8$ & 1 \\
\hline L651 & $*$ & $10 \cdot 3$ & 9.9 & -44.0 & 2 \\
\hline L684 & + & 33.7 & $32 \cdot 3$ & $-11 \cdot 5$ & \\
\hline L2 43 & $*$ & 35.4 & 29.9 & $-12 \cdot 1$ & \\
\hline L1 21 & $\bullet$ & 35.6 & 35.6 & $-7 \cdot 9$ & \\
\hline 1291 & $*$ & 36.1 & 34.4 & $-8 \cdot 4$ & \\
\hline L285 & - & 37.1 & 36.3 & $-6 \cdot 4$ & \\
\hline 1303 & $\sigma$ & 37.3 & $35 \cdot 3$ & -6.9 & \\
\hline 1331 & $\theta$ & 37.4 & 34.9 & $-7 \cdot 1$ & \\
\hline 2366 & $\theta$ & 37.5 & 34.7 & $-7 \cdot 2$ & \\
\hline L388 & $\sigma$ & 37.9 & 37.6 & -4.9 & \\
\hline LS54 & $\sigma$ & 38.6 & 35.4 & -5.9 & \\
\hline 1290 & 0 & 38.9 & 38.5 & -3.6 & \\
\hline 2230 & • & 39.1 & $37 \cdot 3$ & -4.2 & \\
\hline L22 SC & $\boldsymbol{\theta}$ & 39.2 & 36.2 & -4.9 & \\
\hline L352 & $\theta$ & 35.8 & $37 \cdot 2$ & -3.8 & \\
\hline L574 & $\theta$ & 39.9 & 39.9 & $-1 \cdot 9$ & \\
\hline 1360 & $\sigma$ & 39.9 & $3 e \cdot 4$ & $-2,9$ & \\
\hline L618 & $\sigma$ & 40.2 & 40.4 & $-1 \cdot 3$ & \\
\hline L321 & $\sigma$ & 40.2 & 36.9 & -3.8 & \\
\hline 1606 & $\boldsymbol{\sigma}$ & 40.3 & 39.8 & -1.6 & \\
\hline L442 & $\theta$ & 40.4 & $37 \cdot 3$ & $-3 \cdot 3$ & \\
\hline L249 & $\theta$ & 40.4 & 37.6 & -3.2 & \\
\hline L610 & - & 40.5 & 38.9 & $-2 \cdot 1$ & \\
\hline 1148 & $\theta$ & 40.5 & 39.5 & -1.6 & \\
\hline L280 & $\theta$ & 40.5 & 39.3 & $-1 \cdot 8$ & \\
\hline L2 $38 \mathrm{~A}$ & $\boldsymbol{\sigma}$ & 40.7 & 39.3 & -1.7 & \\
\hline $2237 A$ & $\theta$ & 40.7 & 37.8 & -2.7 & \\
\hline L1 34 & $\theta$ & 40.9 & 39.7 & $-1 \cdot 3$ & \\
\hline L190R & $\theta$ & 40.9 & 37.7 & -2.7 & \\
\hline L680 & $\theta$ & 40.9 & 38.4 & $-2 \cdot 2$ & \\
\hline 1312 & $\theta$ & 40.9 & 38.4 & $-2 \cdot 2$ & \\
\hline 1288 & $\theta$ & 41.0 & 39.0 & $-1 \cdot 7$ & \\
\hline L587 & $\theta$ & 41.2 & 36.7 & $-3 \cdot 2$ & \\
\hline 1324 & $\theta$ & 41.2 & 39.1 & $-1 \cdot 4$ & \\
\hline 2685 & $\theta$ & $41 \cdot 3$ & 39.2 & $-2 \cdot 4$ & \\
\hline L100 & $\theta$ & 41.3 & 39.7 & -2.0 & \\
\hline L557 & $\theta$ & 41.3 & 36.8 & -3.0 & -1 \\
\hline L275 & $\theta$ & $41 \cdot 3$ & 40.7 & -.2 & \\
\hline LS70 & $\theta$ & $41 \cdot 5$ & 37.6 & $-2 \cdot 3$ & -2. \\
\hline 2312 & $\theta$ & 41.5 & 40.1 &. .6 & \\
\hline L575 & $\theta$ & 41.5 & 40.2 & -.5 & \\
\hline L261 & $\theta$ & 41.5 & 37.9 & -2.1 & \\
\hline L158 & $\boldsymbol{\sigma}$ & 41.6 & 40.1 & -.5 & \\
\hline L281 & $\theta$ & 41.6 & 37.7 & $-2 \cdot 1$ & -1. \\
\hline L1 57 & $\theta$ & 41.8 & $39 \cdot 3$ &. .9 & \\
\hline II 83 & $\boldsymbol{\sigma}$ & $41 \cdot 8$ & $37 \cdot 5$ & $-2 \cdot 2$ & -1 \\
\hline 1313 & $\theta$ & 41.9 & 40.0 &. .4 & \\
\hline L167 & $\sigma$ & $41 \cdot 9$ & 39.3 & -08 & \\
\hline L232 & $\boldsymbol{\theta}$ & 42.0 & 39.6 & -.6 & \\
\hline L262 & 0 & $4 \bar{\varepsilon} \cdot 2$ & 40.0 & -.2 & \\
\hline 1824 & $\theta$ & $42 \cdot 1$ & 40.5 & $\cdot 2$ & \\
\hline 2559 & $\theta$ & 42.1 & 41.1 & .6 & \\
\hline 2241 & $\theta$ & 42.3 & 39.5 & -.5 & - \\
\hline L25 5 & $\theta$ & 42.3 & 38.6 & $-1 \cdot 1$ & \\
\hline L1 53 & $\theta$ & 42.3 & 39.1 &. .6 & \\
\hline L382 & $\theta$ & $42 \cdot 3$ & 40.1 & .1 & \\
\hline L211 & $\sigma$ & $42 \cdot 3$ & 41.4 & .9 & \\
\hline L259 & $\sigma$ & $42 \cdot 3$ & 40.9 & .6 & \\
\hline L1 66 & $\sigma$ & 42.4 & 39.5 & -.4 & \\
\hline L128 & $\sigma$ & 42.4 & 40.7 & .5 & \\
\hline 1279 & $\theta$ & 42.4 & 39.5 & -4 & \\
\hline L562 & $\theta$ & $42 \cdot 5$ & 40.7 & .6 & \\
\hline L559 & $\theta$ & $42 \cdot 5$ & 38.9 & -.7 & \\
\hline
\end{tabular}

$\triangle$ VG

R. SDR VAR PREPBRTY---TBST INSTRUMBNT---CENDITIENS

\begin{abstract}
.45 15 T TBARING STRENGTH, STANDARD, THWING-BLYKNDERF( SCALE TG 100 .7815 T TBARING STRENGTH, STANDART, THIING-BLITENDERP(SCAIZ TO 100$)$ - 3015 T I3ARING STRBNGTH, STANDARD, THVING-BLIGNDERF( SCALE TO 100) .95 15Y TBARING STRBNGTH, STANDARD: GIVE INSTRUMBNT MAKR, MODEL

2.2215 IBARING SIRENGIH, STANTARD, IBMING-BLNBNDORP( SCALB IO 100)

1.51 15T TYARING STRBNGTH, STANDARD, THVING-BLNBNDERR( SCALB TO 100) 1.0415 T TBARING STRBNGTB, STANDARJ. TEMING-ELJBNDERF(SCALE TO 100 ) 2.2615 TBARING STRBNGTH, STANDARD,THWING-BLISNDERR( SCALE TO 800 ) 1.01 15 L TBARING STRBNGTH, STANDARD, LORBNTZ-WBITRES

1.21 15 T TBAZING SIRBNGTH, STANDARD, THVING-BL MBNDERP( SCALB TO 100 )
\end{abstract}

.89 15 T TBARING STRBNGTH, STANDARD, THFING-ELMGNDERF(SCALB TO 100 ) $1.0915 T$ TIBARING STRENGTH, STANDARD, THWING-BLMBNDERF( SCALS TO 1C0)

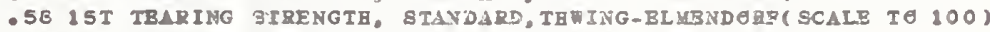
3.05 15 T TBARINO STRZMGTH, RTANDARD.THEING-BLNENDERF( SCALE T 100 )

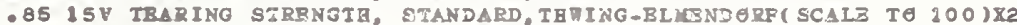

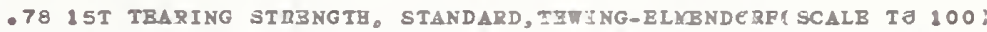
$.7215 C$ TBARING STRZNGIH, STANDARD, TEWING-ZIMGNDERP (HOAIR CLAMP) 2.0015 TBARING STRENG II S STANDARD, THWING-ZIMBND ERF( SCALB TO 100 ) 1.01 IST TEARIMG STRBNGTH, STA!DARD, THWING-BLKBNDERF(SCAIE TO 100 .6715 T TBARING STRBNGTH, STANDARD, TBIING-ELHBNDERFI SCALB TO 100

.68 15 T TBARING STRENGTH, STANDARD, THMING-BLMBNDERP( SCALB TE 100 ) .9815 T TBARING STRBNGTH, STANDARD, THWING-ELKCSDERF(SCALE TE 100 ) .89 15R TBARING STRBNGTE, STANDARD, THFING-BLIGNDERF, DIGITAL RELDEUT 0915 T TBARING STRBNGIB, STINDARD, TRVING-BLYZNDERP( SCALB TO 100$)$ 1.01 15B TBARING STRENGIH, STANDARD, THNING-BLLIBNDERR, AMIIBNT CEND.

.49 15 T TBARING STRENGTH, SILNDARD,THRING-BLIBNDERP(SCALB TE 100) $7415 L$ TRARING STRBNGTH, STANDARD, LERSYTZ-TBTTRBS

-95 15 T TBARING STRENGTE, STANDARD, TEPING-BLICNDERP( SCALE TE 100 ) $.8815 T$ TRARING STRBNCTH, STANDARD, IBTING-BLNBNDERF( SCALB TO 100) $.6315 \mathrm{C}$ TEARING STRBNGTB, S'TANDARD, THIING-BLIENDERF (T.AIR CLAMP)

$.5315 C$ TBARING STRBNGTH, STANDARD, THWING-BLYBNDGRF (W.AIR CLAMP) .8815 I TBARTNG STRBNGTH, STANDARD, TBWIMG-BLNGNDERP(SCALB TO 100) $1.1 C 15 T$ TEARING STRBNGTI, STANDARD, IBMING-3LKBNDERF(SCALD TE 100) .95 150 IEARING STRBNGTH, STANDARD, IBWING-BLICNDFRF, $A$ IR CLAMP, DIGITL 1.27 15I TEAZING STRBNCTE, STANDARD, TIWIFG-EIMBNDERP(SCAIB TE 100)

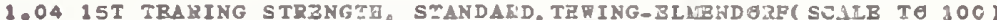
1.41 $15 T$ TBARINO STRBNGTY, STINDARD, TEFING-B?,UBNDERF(SCALB IE 100) .6115 TBARING STRBNGTH, STANDARD, T.M.MIREIBLD(APPITA-BLLENDORF) $1.5515 T$ TBARING SIRBNGTH, STINDAKD, IBTING-BLMENDERP(SCALE TC 200 )

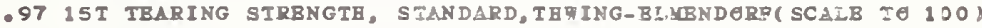

$.9715 T$ TEARING STRBNGTH, STANDARD, THWING-ELMBNDERF (SCALB TE 100) 1.40 $15 T$ TBARING STRBNGTH, STANDARD, THWING-BILENDORF( SCALE TO 100) $.7715 L$ TBARING STRBNGTE, STANDARD, LORZNTZ-METTRBS

$.8215 T$ TBARING STRSNGTY, STANDARD, THWING-BLMBNDERP(SCALB TE 100) $2.0015 R$ TBARING STRBNGTB, STANDARD, TBWING-BLMBNDERF, DIGITAL READEUT

$6125 T$ TBARING STRBNGTH, STANDARD, THWING-BLMBNDERF( SCALZ TE 100) 1.0315 I TBARING STRBNGIH, STANDARD. IHWING-BL IBNDERP( SCALE TO 100) $6215 T$ TBARING STRBNGTE, STANDARD, THVING-ELMBNDERP( SCALE TO 100 ) $.9615 L$ IBARZNG STRBNGTH, STANDARD, LERBNTZ-WBTTRBS

1.0915 C TBARING STRBNGTH, STANDARD, THWING-BLMTNDERF (W.AIR CLAMP)

1.32 $15 T$ TBARING STRENGTB, STANDARD, IBDING-BL IRNDERF( SCALB TE 100) . 8215 T TBARING STRENGTH, STANDARD, THEING-ELIENDGRF( SCALE TO 100 ) $1.6215 T$ TBARING STRENGTH, STANDARD, IBWING-BLIBNDORP( SCALB TO 100 ) $1.0015 T$ TBARING STRBHGIH, SIANDARD, IBRING-BLIBNDERF( SCALE T 100 ) $.8715 T$ TBARING STRBNGTH, STANDARD, THWING-ELLBNDERF(SCALB TO 100)

$.3615 T$ TEARING STRENOTH, STANDARD, THEING-ELLENDERF( SCALB TE 100 ) .78 15C IBARING STRBNGIB, STANDARD, IRWINO-BLIENDERF (V.AIR CLAMP) .90 $15 T$ TBARING STRBNGTH, STANDARD, THVING-BLMBND ERP( SCALB TE 100 ) $1.1015 \mathrm{R}$ TBARING STRZNGTH, STANDARD, TH ING-BLLBNDERP, DIGITAL READEUT 1.06 isT TBARING STRBNGTE, STANDARD, IBGING-BLNBNDERP( SCALB TO 100 )

$8215 T$ TIARING STRBNGTH, SIANDARD, TIVING-BL IRNDEPP(SCALB IO 100 ) - 71 15T TRARING STRENGTH, STANDARD, TBRING-BLLENDORP(SCALB TO 100) $1.2215 T$ TBARING STRBNGTB, STANDARD, TH ING-BLLENDERF( SCALB TӨ 190 $1.6615 T$ TBARING SIRZNGTH, STANDARD, IHVING-BLHBNDERP( SCALB TO 100) $1.0615 T$ TBARING STRBNGTH, STANDARD, TEITHG-BLIRNDERF( SCALB TO 100) 
TAFPI STANDARD IAI 4 TS-65. ANY LAKB BLMBNDORP WITB DEBP CUTOUT IS STANDARD FOR THIS ANALYSIS

\begin{tabular}{|c|c|c|c|c|c|c|c|c|c|c|c|}
\hline \multicolumn{2}{|l|}{ LAB } & \multicolumn{2}{|c|}{ MBANS } & \multicolumn{2}{|c|}{ COORDINATBS } & $\Delta V_{G}$ & \multirow[b]{2}{*}{ VAR } & \multirow{2}{*}{\multicolumn{2}{|c|}{ PROPBRTY - - IBST }} & \multirow[b]{2}{*}{ INST RUMBNT $-\infty$ CENDITIENS } & \\
\hline CEDB & $\mathbf{F}$ & $\mathrm{B} 25$ & E85 & MAJOR & MINER & R. SDR & & & & & \\
\hline L248 & $\theta$ & 42.6 & 40.3 & .4 & $\bullet 1$ & .85 & $15 \mathrm{~J}$ & TBAR ING & SI RENGTH, & I & \\
\hline 2688 & $\theta$ & 42.7 & 42.8 & $2 \cdot 2$ & 1.8 & .73 & $15 \mathrm{~T}$ & TBARING & STRBNGTH, & STANDARD, TBVING-ELNBNDORP ( SCALE & IE 1001 \\
\hline L105 & $\theta$ & 42.7 & 40.5 & .6 & .2 & 1.34 & $15 T$ & TBARING & STRENGTB, & SIANDARD, TB ING-BINGNDORF( SCALB & TO 100 ) \\
\hline L243 & $\theta$ & 42.8 & 39.6 & .0 & .5 & .70 & $15 \mathrm{~T}$ & TBARING & STRBNGTH. & STANDARD, THWING-BLLBNDERF( SCALB & To 1001 \\
\hline L580 & $\theta$ & 42.8 & $38 \cdot 3$ & $\because 9$ & $-1 \cdot 5$ & .42 & $15 T$ & TBARING & STRENGTB, & STANDARD, THWING-ELMBNDERF( SCALE & $T 0100)$ \\
\hline L1 85 & $\theta$ & $42 \cdot 8$ & $42 \cdot 3$ & 1.2 & .7 & .91 & $15 \mathrm{~T}$ & TBARING & STRBNGTB, & STANDARD, IBWING-BLMBND GRF ( SCALB & T0 100) \\
\hline 1206 & $\theta$ & $42 \cdot 9$ & 39.5 & -.0 & -07 & .77 & $15 R$ & TBAR I NG & STRBNGIB, & STANDARD, I BVING-ELIOBNDGRP, DI G I I & $\triangle L$ LBADEUT \\
\hline L126 & $\theta$ & 42.9 & 41.8 & 1.6 & 2.0 & 1.24 & $15 \mathrm{~T}$ & TBARING & SIRBNGTB, & STANDARD, THVING-BL VENDERPI SCALB & IO 100$)$ \\
\hline L 328 & $\theta$ & $42 \cdot 9$ & 40.1 & .5 & -.2 & .28 & $15 \mathrm{~T}$ & TBARING & STRENGTB, & STANDARD, THE ING - ELNGNDERP ( SCALB & I0 1001 \\
\hline 1274 & $\theta$ & $42 \cdot 9$ & $39 \cdot 7$ & .2 & -.5 & .48 & $15 \mathrm{~T}$ & TBARING & STRBNOTB, & STANDARD, TBVING - ELLBNDGRP ( SCAIB & To 100$)$ \\
\hline L345 & $\theta$ & 42.9 & $42 \cdot 1$ & 1.9 & 1.2 & 1.82 & $15 \mathrm{~T}$ & TBARING & STREN3TH, & ST $\triangle N D A R D, T B W I N G-B L M B N D \in R P(S C A L E$ & To 1001 \\
\hline L2є4 & $\mathbf{x}$ & 42.9 & 35.7 & -2.6 & -3.4 & 1.34 & $15 \mathrm{~T}$ & TBARING & STRBNGIB. & STANDARD, IBWING - BLMBNDERP ( SCALB & I0 1001 \\
\hline L237B & $\theta$ & 43.0 & 40.6 & .9 & .0 & .70 & $15 \mathrm{~T}$ & TBARING & STRBNOIB. & STANDARD, I EW ING-ELIOBNDERF( SCALB & TO 100$)$ \\
\hline L1 89 & $\boldsymbol{\theta}$ & 43.0 & 41.8 & $1 \cdot 7$ & .9 & .88 & $15 \mathrm{~T}$ & IBARING & STRBNGTB, & STANDARD, IBVING - ELIOBNDGRF( SCALB & I0 100$)$ \\
\hline $\mathbf{L 3 0 9}$ & $\theta$ & 43.1 & 40.4 & .8 & -.2 & .82 & $15 \mathrm{~T}$ & TBAEING & STRBNGTB. & STANDARD, TBVING = EL ICBN DERF( SCALE & To 1001 \\
\hline $2257 \mathrm{C}$ & $\theta$ & 43.1 & 41.9 & 1. 8 & .9 & 1.03 & $15 \mathrm{C}$ & TBARING & STRENGTB, & IBWING-BLYBNDERP & - AIR CLAMP) \\
\hline $1290 \mathrm{C}$ & $\theta$ & 43.1 & 39.5 & .2 & -.8 & .67 & $15 \mathrm{~T}$ & TBARING & STRBNGTB, & ST NNDARD, TBWING-ELIBNDERP( SCALB & I0 1001 \\
\hline 2217 & $\theta$ & 43.2 & $41 \cdot 5$ & 1.6 & $\cdot 5$ & .67 & $15 T$ & TBARING & STRBNGTB, & STAND $\triangle R D$, TH QING - BL MBNDERF( SCAIB & I0 1001 \\
\hline 1278 & $\theta$ & 43.2 & 40.9 & 1.2 & $\cdot 1$ & .93 & $\$ 5 \mathrm{~T}$ & TBARINO & BTRBNOTB, & STANDARD, TBWING-BLMBNDERF( SCALB & IO $1(0)$ \\
\hline $\mathbf{L 3 4 4}$ & $\theta$ & 43.2 & 40.4 & .9 & $\leadsto 3$ & 1.12 & $15 \mathrm{C}$ & TBARING & STRBNGIB, & IEVING-ELMBNDGRF ( & $\triangle I R$ CLAMP) \\
\hline $\mathbf{L 2 1 9}$ & $\theta$ & 43.2 & $41 \cdot 3$ & 2.5 & .4 & 1.10 & $15 I$ & TEARING & STRENOTB, & LORENTZ-WBTIRBS & \\
\hline LI B2A & $\sigma$ & $43 \cdot 3$ & 38.3 & -.06 & $-1 \cdot 8$ & 1.29 & 154 & TBARING & STRBNGIB, & STANDARD, & \\
\hline 2679 & $\theta$ & $43 \cdot 3$ & 38.3 & -.06 & $-1 \cdot 8$ & .94 & $15 \mathrm{~T}$ & TBARING & SIRBNGTB. & STANDARD, IBW ING - BLIBNDERF( SCALB & I0 1001 \\
\hline 1225 & $\theta$ & $43 \cdot 3$ & $43 \cdot 4$ & 3.0 & $1 \cdot 8$ & .68 & $15 \mathrm{~T}$ & TBARING & STRBNGTB. & STANDARD, I BWING-BL KBNDERFI SCAIB & I0 100$)$ \\
\hline $\mathbf{L 5 5 8}$ & $\theta$ & $43 \cdot 3$ & $41 \cdot 7$ & $1 \cdot 8$ & $\cdot 7$ & 2.06 & $15 \mathrm{~T}$ & TBARING & STRBNGIB, & STANDARD, THWING - BLLBNDGRFI SCALB & IO 100$)$ \\
\hline L226B & $\theta$ & 43.3 & 40.0 & .7 & -.6 & 1.35 & $15 \mathrm{~T}$ & TBARING & ST RBNGTH, & ST $\triangle N D \triangle R D, T H W I N G=B L W B N D O R F(S C A L B$ & I0 100$)$ \\
\hline LL 62 & $\theta$ & $43 \cdot 3$ & 40.5 & 1.1 &. .2 & .89 & $15 \mathrm{~T}$ & IBARINO & ST RBNGTH, & ST $\triangle N D A R D$, I HW I NG - BL)KBNDERP ( SCALB & I 100$)$ \\
\hline L597 & $\theta$ & 43.5 & 40.1 & .9 &. .6 & .61 & $15 \mathrm{~T}$ & TBARING & STRBNGTB, & STAND $\triangle R D, T B W I N G-B L L B N D G R F(S C A L B$ & IO 1001 \\
\hline L257A & $\theta$ & 43.5 & 41.6 & 1.9 & .4 & .73 & $15 \mathrm{C}$ & TBARING & STRBNGTH, & STANDARD, TBWING-BL MBNDORF ( & AIr CLANP) \\
\hline L257B & $\theta$ & 43.5 & 42.0 & 2.2 & .7 & .99 & $15 \mathrm{C}$ & TBARING & STRBNGTH, & IBTING - ELMBND GRF & - AIR CLAMP) \\
\hline L242 & $\cdot$ & 43.6 & 41.1 & 1.6 & $\cdots 1$ & 1.12 & 150 & TEARING & STPBNGTE, & AUSTRALIAN EFT, CO. & \\
\hline $\mathrm{L} 273$ & $\theta$ & 43.6 & 38.4 & -.3 & -2.0 & 1.06 & $15 \mathrm{I}$ & IBAR I NG & STRBNGTB, & STAND $\triangle R D$, IBWING-BL NBNDORF( SCALB & To 1001 \\
\hline L315 & $\theta$ & 43.6 & 39.8 & .7 & -1.0 & 1.22 & $15 \mathrm{~T}$ & TBARING & STRBNGTB, & STANDARD, THEING-BLMBNDORP( SCALB & To 1001 \\
\hline L35,6M & $\theta$ & 43.7 & 44.0 & 3.8 & 1.9 & 3.25 & $15 \mathrm{~T}$ & TBARING & SIRBNGTB, & STANDARD, THWING-BLIBNDERF( SCALB & 101001 \\
\hline L291 & $\theta$ & 43.8 & $42 \cdot 5$ & 2.7 & .8 & 1.55 & 154 & IBARING & STRBNGIB, & STANDARD, & \\
\hline 1223 & $\theta$ & 43.8 & 40.3 & 1.2 & -.7 & .71 & $15 \mathrm{R}$ & TBA RING & ST RBNGTH, & STANDARD, TBWI NG-EL MBND $Q R F$, DI G I TA & AL RBADEUT \\
\hline L122 & $\theta$ & 43.8 & 43.3 & 3.4 & 1.4 & .97 & $15 \mathrm{c}$ & IBARING & STRBNGTB, & THEI NG-BLMBND ERP ( & AIR CLAMP) \\
\hline L212 & $\theta$ & 43.9 & 40.7 & 1.6 & -.5 & $1 \cdot 25$ & $15 \mathrm{~T}$ & TBAPING & SIPBNGTE, & ST $\triangle N D \triangle R D, T H \cup I N G$ - ELMBNDERF( SCALE & I0 100) \\
\hline L139 & $\theta$ & 43.9 & 41.8 & 2.4 & .2 & .62 & $15 \mathrm{I}$ & TBARING & STRBNOTB, & STAND $\triangle R D, T B W I N G$ - BLMCBNDG RF( SCALB & To 1001 \\
\hline 1676 & $\theta$ & 44.0 & 40.1 & 1.2 & -1.0 & 1.57 & $15 \mathrm{~T}$ & TBARING & ST RBNOTB, & STANDARD, TBEING - BLICBNDGRF( SCALB & Io 100$)$ \\
\hline 1107 & $\theta$ & 44.0 & 41.7 & 2.4 & .1 & .72 & $15 \mathrm{~T}$ & TBARING & STRBNGTB, & ST ANDARD, TBVING -BL NBNDERP( SCALE & TO 1001 \\
\hline $\mathbf{L 5 6 7}$ & $\theta$ & 44.1 & 39.6 & 1.0 & $-1 \cdot 5$ & 1.70 & $15 \mathrm{C}$ & TBARING & SIRENOTE, & IBUING-BLIB NDERP ( $\bar{*}$ 。 & - AIR CLAMP) \\
\hline 1295 & $\mathbf{0}$ & 44.1 & 39.7 & 1.1 & -1.4 & 1.19 & $15 \mathrm{C}$ & TEARING & STRBNGTB, & TBEING-ELIBNDORP $($. & AIR CLAMF) \\
\hline L1 $<3$ & $\boldsymbol{\sigma}$ & 44.2 & 39.4 & .9 & $-2 \cdot 7$ & 1.11 & $15 \mathrm{~T}$ & TBARINO & BTRENOTB, & STANDARD, TBW ING - BLMBNDGRF ( SCALB & To 100$)$ \\
\hline $\mathbf{L 3 3 6}$ & $\boldsymbol{0}$ & 44.3 & 41.2 & 2.2 & .4 & .95 & $15 \mathrm{~T}$ & TBA ING & STRBNGTB. & STANDARD, THEING - BLLBNDERF( SCALB & To 1001 \\
\hline L2:3 & - & 44.3 & 42.0 & $\cdot 7$ & $\cdot 1$ & .95 & $15 \mathrm{~T}$ & TBARING & STRENGTH, & BT $\triangle N D \triangle R D, T B=I N O-B L M B N D E P(S C A L B$ & TO 100$)$ \\
\hline LI $73 B$ & $\boldsymbol{\theta}$ & 44.5 & 41.3 & $2 \cdot 5$ & -.5 & .86 & $15 T$ & IBARING & BTRBNGIB, & SIANDARD, THWING-BLMENDERF ( SCALB & To 100$)$ \\
\hline L581 & $\theta$ & 44.6 & 40.5 & 2.0 & 1.2 & .75 & 150 & TBARING & STRBNGTE, & STANDARD, TBWING-BLIRNDERF, $\triangle I R C L$ & LAM,DIGITL \\
\hline L241 & $\boldsymbol{\theta}$ & 44.7 & 43.1 & 3.8 & .6 & .69 & $15 \mathrm{I}$ & TBARING & SIRBNGIB, & STANDARD, IBW ING - BLIBNDORF ( SCALB & TO 1001 \\
\hline L254 & $\theta$ & 44.9 & 40.8 & $2 \cdot 4$ & $-1 \cdot 2$ & 1.00 & $15 \mathrm{~T}$ & TBARING & STRBNGTB, & STANDARD, TBVING - BLMBNDORF( SCALB & T0 1001 \\
\hline 2600 & $\theta$ & 44.9 & 40.7 & 2.3 & $-1 \cdot 3$ & .70 & $13 \mathrm{I}$ & TBA ING & ST RBNGTH, & ST $\triangle N D \triangle R D$, THW ING - BLMBNDORF( SC $\triangle L B$ & To 100$)$ \\
\hline L194 & $\theta$ & $4 \varepsilon_{.} 1$ & 43.6 & 4.4 & .7 & .86 & $15 \mathrm{I}$ & TBAP I NG & SIRBNGIB, & SIANDARD, I BW ING-BLMBND ORF( SCALB & I0 100$)$ \\
\hline L565 & $\theta$ & 45.3 & 43.9 & 4.8 & - 8 & 1.04 & $15 \mathrm{I}$ & TBARING & STRBNGIH, & STANDARD, TBVING - BLMBNDORP( SCALE & I6 100$)$ \\
\hline 2244 & $\theta$ & 45.3 & 43.8 & 4.7 & .7 & 1.05 & $15 \mathrm{C}$ & TBARING & SIRBNGTH, & IBWING-BLIBNDORP ( & $\Delta I R$ CLAMP) \\
\hline $\mathbf{L} 484$ & $\theta$ & 45.3 & 42.5 & 3.9 & -.2 & 1.48 & $15 \mathrm{I}$ & TB $\triangle R$ I NG & ST RBNGTH, & 8TANDARD, I B ING - BLIGBNDERP ( SCALB & $10100)$ \\
\hline 1299 & $\bullet$ & 45.4 & 42.7 & 4.1 & .1 & .81 & $25 \mathrm{~V}$ & TBARING & SIRBNGTH, & ST $\triangle N D \triangle R D, T H \|$ ING - BL MBNDGRP( SCALB & TO $1 0 0 \longdiv { \times 2 }$ \\
\hline 22501 & + & $4 \Xi_{\bullet} 4$ & 44.3 & 5.2 & 1.0 & .98 & $15 \mathrm{~B}$ & TBARI NO & SIRB NGIB, & LHEMARGY, $20 \mathrm{C}, 65 \%$ & RH \\
\hline $\mathbf{1 5 5 4}$ & $\theta$ & 45.7 & 43.2 & 4.6 & .0 & 1.10 & $15 \mathrm{C}$ & TEAR ING & SIRBNGTH, & IHEING-BLMBNDERP ( & $\triangle I R$ CLAMP) \\
\hline L566 & $\theta$ & 45.7 & 40.8 & 3.0 & -1.7 & .83 & $15 \mathrm{~T}$ & IBARING & STRBNGTH, & STANDARD, THWING-BLLBNDERP( SCALB & IO 100$)$ \\
\hline $\mathbf{L 2 3 6}$ & $\theta$ & 45.7 & 42.7 & 4.3 & $\because 4$ & 1.11 & $15 \mathrm{~T}$ & TBARING & STRBNGTH, & STANDARD, TBWING - BLMBNDGRPI SCALB & TO 100$)$ \\
\hline L228 & - & 46.3 & $40 \cdot 7$ & 3.2 & $-2 \cdot 2$ & 1.44 & $15 \mathrm{~T}$ & TBARING & STRBNGTE, & STANDARD, THVING - BL KBNDERF( SCALB & TO 100$)$ \\
\hline L576 & 6 & 46.4 & 43.9 & 5.6 & .0 & .99 & $15 \mathrm{~T}$ & IEAR ING & STRBNGTH. & STANDARD, THE ING - BLMBNDERP( SCALB & To 100$)$ \\
\hline L390 & $\theta$ & 46.7 & 43.5 & $5 \cdot 5$ & -.4 & .71 & $15 \mathrm{I}$ & TBARINO & STRBNG TE, & STANDARD, IBW ING - B LMBNDORP( SCALB & To 100$)$ \\
\hline L182T & $\theta$ & 46.9 & $43 \cdot 8$ & 5.9 & $\ldots 4$ & .83 & $15 \mathrm{~T}$ & IBARING & STRBNGTH, & STANDARD, THE ING - BLMBND ERP ( SCALB & To 100$)$ \\
\hline L131 & - & 47.7 & 45.3 & 7.5 & .1 & .61 & 154 & IBARING & STRBNGTE, & STANDARD, & \\
\hline
\end{tabular}


TAPPI STANDARD I414 T9-65, $\triangle N Y$ MARB BLKBNDERP VITB DEBP CUIGUT IS SIANDAED POR IBIS ANALYSIS

\begin{tabular}{|c|c|c|c|c|c|c|c|c|c|c|}
\hline LAB & & MB. & & Coerd & NATBS & $\Delta$ VG & & & & \\
\hline CODE & $\mathbf{F}$ & Kas & B85 & MAJER & MINER & R. SD R & VAR & PREPBI & RTY $=-$ TBST & INSTRUMBNT - - CONDITIENS \\
\hline 1652 & * & 49.9 & 48.8 & 11.5 & 1.1 & 3.50 & $15 \mathrm{C}$ & TBAR ING & STRBNGTH, & THWING-BLYBNDERF (M.AIR CLAMP) \\
\hline 1207 & * & 51.2 & 49.5 & 12.9 & .6 & $\therefore .35$ & 158 & IBAR I NG & STRBNGTH, & STANDARD, TH ING-BLIENDERF DIGITAL READGUT \\
\hline L150 & 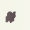 & 53.0 & $50 \cdot 3$ & 14.8 & .00 & .68 & $\$ 5 T$ & TBAR ING & STRBNGTE, & STANDARD, THWING-ELUBNDORF( SCALE TO 100) \\
\hline 2158 & 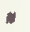 & 53.1 & 47.7 & 13.0 & -1.9 & 1.45 & $15 \mathrm{C}$ & TBARING & STRENGTH, & STANDARD, THWING-BLKBNDORF (T.AIR CLAUP) \\
\hline L604 & . & 55.5 & 54.9 & 22.7 & $-1 \cdot 3$ & 3.56 & $15 \mathrm{~T}$ & TBARING & STRBNGTE。 & STANDARD, THWING-BLIENDERP( SCALB TO 100 ) \\
\hline 1622 & . & 63.7 & 60.8 & 29.8 & -.1 & 1.74 & $15 T$ & TBARING & STRBNGTH, & STANDARD, THWING - BLNENDERF( SCALB TO 100) \\
\hline GMBAN & & $\begin{array}{l}42.4 \\
95 \times\end{array}$ & $\begin{array}{l}40.0 \\
\text { LIPSB : }\end{array}$ & $7 \cdot 3$ & 2.3 & $\begin{array}{l}1.00 \\
\text { WITH }\end{array}$ & $\mathbf{G A M}$ & $\mathbf{L}-44$ & EGREES & \\
\hline
\end{tabular}


TEARING STRENGTH, DEEP CUTOUT

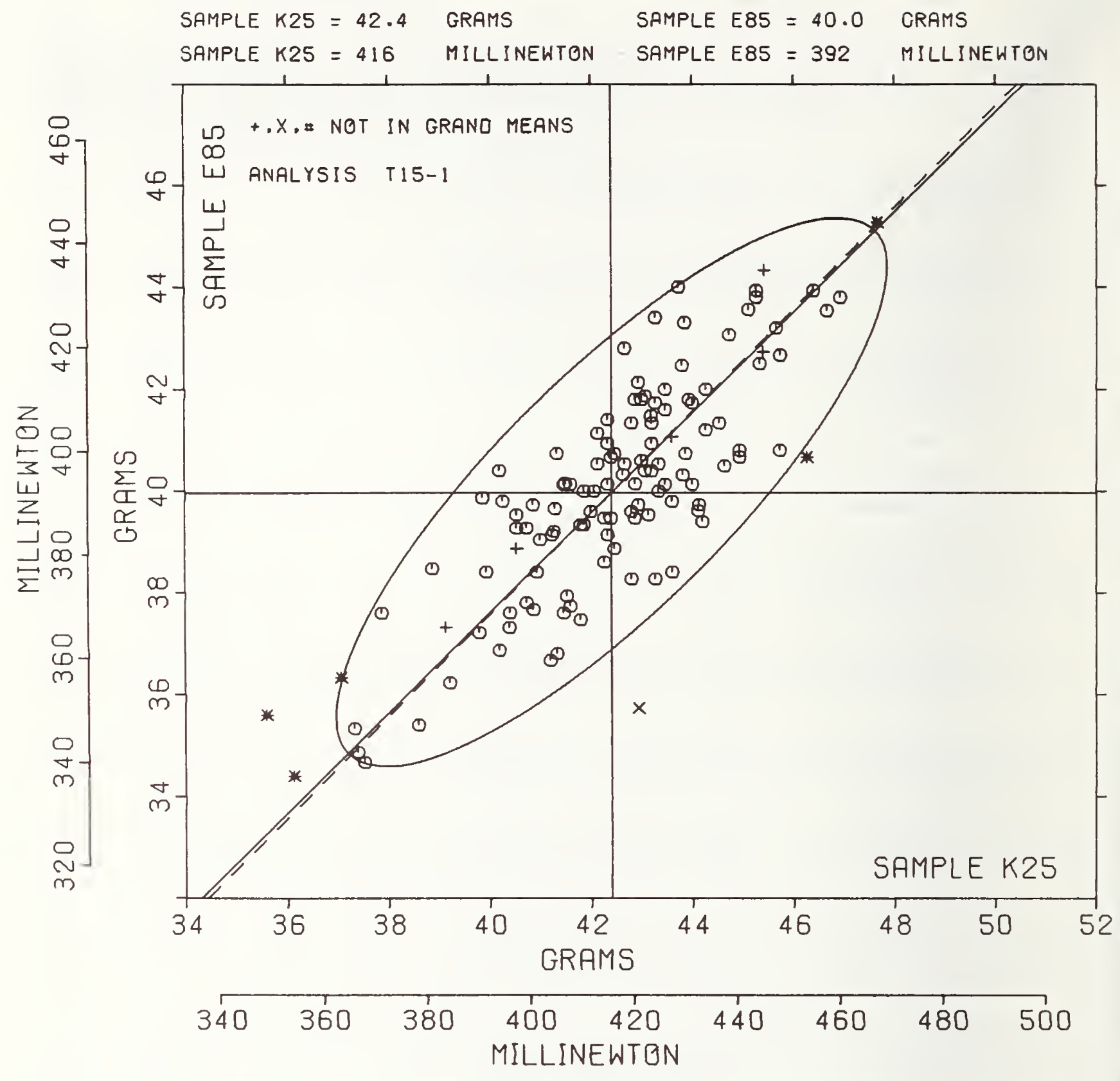


TAPPI STANDARD T414 TS-65, TBWING-BLIGNDORP WITBOUT DBHP CUTOUT IS STANDARD FOR IBIS ANALYSIS

\begin{tabular}{|c|c|c|c|c|c|c|c|c|c|c|c|c|c|}
\hline \multirow{3}{*}{$\begin{array}{l}\mathrm{LAB} \\
\mathrm{CODB}\end{array}$} & \multirow{3}{*}{$\begin{array}{c}\text { SAMPLB } \\
\text { K } 35 \\
\text { MBAN }\end{array}$} & \multicolumn{4}{|c|}{ PRINTING } & \multirow{3}{*}{$\begin{array}{c}\text { SAMPLB } \\
\text { J } 42 \\
M B A N\end{array}$} & \multirow{3}{*}{$\begin{array}{l}86 \text { GRAM } \\
\text { DSV }\end{array}$} & \multicolumn{3}{|c|}{ PRINI ING } & \multirow{3}{*}{$\begin{array}{r}\text { TBST } \\
\text { VAR }\end{array}$} & \multirow{2}{*}{$D_{0} \cdot$} & \multirow{2}{*}{15} \\
\hline & & 105 GEAMS & 3 PBR & SQUARE & & & & S PER & SQUARB $M$ & MBTBR & & & \\
\hline & & DE V & N. DEV & SDR & R. SDR & & & N. DBV & SDR & R. SDR & & $\mathbf{F}$ & $L \Delta B$ \\
\hline L1 22 & 125.0 & 6.1 & .62 & 3.8 & .95 & 66.0 & 2.8 & .34 & 4.0 & 1.39 & $17 \mathrm{~N}$ & $\theta$ & L122 \\
\hline L148 & 118.7 &. .2 & -.02 & 3.8 & .93 & 57.1 & -7.1 & -1.32 & 2.6 & .90 & $17 \mathrm{~N}$ & $\theta$ & Lis 48 \\
\hline 2231 & 120.0 & 1.1 & .11 & 2.1 & .52 & 66.5 & $2 \cdot 3$ & .42 & 1.6 & .57 & $17 \mathrm{~N}$ & $\theta$ & L231 \\
\hline 1234 & 130.1 & $11 \cdot 2$ & 1.15 & 5.2 & 1.29 & 70.4 & 6.2 & 1.15 & 2.7 & .95 & $17 \mathrm{~N}$ & $\sigma$ & L234 \\
\hline$\llcorner 267$ & 135.3 & 16.3 & 1.68 & 6.4 & 1.57 & 73.1 & 8.9 & 1.66 & 4.0 & 1.39 & $17 \mathrm{~N}$ & $\theta$ & L267 \\
\hline 2269 & 123.9 & 5.0 & .51 & 4.0 & 1.00 & 67.8 & 2.9 & .55 & 3.2 & 1.10 & $17 N$ & $\theta$ & L269 \\
\hline L302A & 119.2 & .3 & .03 & 4.4 & 1.10 & 64.2 & .0 & .00 & 2.7 & .92 & $17 \mathrm{~N}$ & $\theta$ & L301A \\
\hline L301B & 117.5 & $-1 \cdot 4$ & -.15 & 3.7 & .91 & 65.7 & $1 \cdot 5$ & .27 & 2.7 & .94 & $17 \mathrm{~N}$ & $\boldsymbol{\theta}$ & L301 B \\
\hline 2308 & 125.9 & 6.9 & .71 & 5.2 & 1.29 & $64 \cdot 3$ & .8 & .08 & 3.0 & 1.04 & $17 N$ & $\theta$ & L308 \\
\hline 1326 & $111 \cdot 3$ & -7.7 & -.79 & 3.3 & .82 & 58.7 & $-5 \cdot 5$ & -1.02 & 2.8 & .99 & $17 \mathrm{~N}$ & $\theta$ & 1326 \\
\hline 2339 & 100.8 & -18.1 & -1.87 & 3.1 & .77 & 55.2 & -9.0 & -1.67 & 2.5 & .90 & $17 \mathrm{~N}$ & $\theta$ & L339 \\
\hline 1372 & 104.1 & -14.9 & -1.53 & 4.1 & 1.02 & 58.3 & -5.9 & -1.10 & 2.3 & .78 & $17 \mathrm{~N}$ & $\theta$ & 1372 \\
\hline$L 393$ & 114.5 & -4.4 & .045 & 3.4 & .84 & 68.0 & 3.8 & .71 & 3.3 & 1.14 & $17 \mathrm{~N}$ & $\theta$ & L393 \\
\hline
\end{tabular}

GR. MBAN - 118.9 GRAMS

SD MBANS - 9.7 GRAMS

AVERAGB SDR -

GR. MEAN -1166.5 MILLINEWTEN GRAND MBAN - 64.2 GRAMS
SD OF MBANS - 5.4 GRAMS 4. O GRAHS

\section{GRAND}

Best values: $\begin{array}{rr}\mathrm{K} 35 & 119 \pm 16 \text { grams } \\ \mathrm{J} 42 & 65 \pm 9 \text { grams }\end{array}$
Plense see the diagram on the inslde of the back cover of thlo report ahlch shows how to distingulsh between an Elmendorf tear tegter 1 th DEEP CJTEUT and an older tegter ith Ne cutejt.

TAPPI STANDARD T414 TS-65. TBVING-BL UENDERF WITBEUT DERP CUTEUT IS STANDARD FER THIS ANALYSIS

\begin{tabular}{|c|c|c|c|c|c|c|c|c|c|c|c|c|c|}
\hline \multirow{2}{*}{$\begin{array}{l}\mathrm{LAB} \\
\mathrm{C} D \mathrm{DB}\end{array}$} & \multirow[b]{2}{*}{$\mathbf{F}$} & \multicolumn{2}{|c|}{ MBANS } & \multicolumn{2}{|c|}{ COORD I NAT BS } & \multicolumn{8}{|l|}{ AvG } \\
\hline & & $\mathbf{3 5}$ & 342 & MAJER & MINER & R. SDR & VAR & \multicolumn{2}{|c|}{ PREPBRTY - - TBST } & \multicolumn{4}{|c|}{ INSTRONENT - - CENDIT IENS } \\
\hline L339 & $\theta$ & 100.8 & 55.2 & $-20 \cdot 3$ & .1 & .83 & $17 \mathrm{~N}$ & TBAR I NG & STRBNGTB & No & $\cot T$ & EUT. ? & TBWI NG-BL KBND ERF \\
\hline$L 372$ & 0 & 104.1 & 58.3 & $-16 \cdot 0$ & $1 \cdot 3$ & .90 & $17 \mathrm{~N}$ & TBARING & STRBNGTB & NO & CUT & OUT, & I घWI NG -BLMBND ORF \\
\hline L326 & $\theta$ & 111.3 & 58.7 & -9.3 & -1.5 & .90 & $17 \mathrm{~N}$ & TBARING & STRBNGTB, & No & CUT & өठT, & TBW I NG-BL MBND E RF \\
\hline L393 & $\theta$ & 114.5 & 68.0 & $-2 \cdot 3$ & 5.4 & .99 & $17 \mathrm{~N}$ & TBAR ING & STRBNGTB, & ne & CUT & EUT, ? & IBWI NG-BL MBNDERP \\
\hline L301B & $\theta$ & 117.5 & 65.7 & .66 & 2.0 & .93 & $17 \mathrm{~N}$ & IBAR I NO & STRBNOTB, & No & CUT & बUT, & I BW I NG - BLYB ND ERF \\
\hline 2148 & $\theta$ & 118.7 & 57.1 & -3.4 & $-6 \cdot 3$ & .91 & $17 \mathrm{~N}$ & TBARING & STRBNGTB, & Ne & $\cot T$ & өUT, & TBWI NG-BL MEN DERF \\
\hline 23014 & 0 & 115.2 & 64.2 & .2 & .1 & 2.01 & $17 \mathrm{~N}$ & TBAR ING & STRB NGTB, & NO & COT & ธบT. & I BW I NG - BL NBB N D ERF \\
\hline L231 & $\theta$ & 120.0 & 66.5 & 2.0 & 1.6 & .54 & $17 \mathrm{~N}$ & TBAR ING & BTRENGTB & No & CUT & อUT. & TBVING-BLKBNDERF \\
\hline L269 & 6 & 123.9 & 67.1 & 5.8 & .4 & 1.05 & $17 N$ & TBARING & STRBNGTB, & Ne & CUT & ยบT. & IBW ING-BL MBN DERF \\
\hline L122 & $\theta$ & 125.0 & 66.0 & 6.2 & -2.1 & 1.17 & $17 \mathrm{~N}$ & TBARING & STRBNGTB, & No & CUT & OUT, & I BW INO-BL LBN DO RP \\
\hline L 308 & $\theta$ & 125.9 & $64 \cdot 3$ & 6.2 & -3.0 & 1.26 & $17 \mathrm{~N}$ & TBARINO & STRBNGTH, & $\mathbf{N} \boldsymbol{\theta}$ & $\mathrm{COT}$ & өOT. & THW I NG - BLMBND ORF \\
\hline L234 & $\theta$ & 130.1 & 70.4 & 12.8 & .6 & 1.12 & $17 \mathrm{~N}$ & TEARING & STRB NOTE, & NO & cot & өUT. & IBG I NG-BL MB NDERP \\
\hline 2267 & $\theta$ & 135.3 & 73.1 & 18.6 & .7 & 1.48 & $17 \mathrm{~N}$ & IBAR ING & STRBNGTB, & NO & COT & बUT, ? & IB ING-BLLENDORF \\
\hline \multirow{2}{*}{\multicolumn{2}{|c|}{ GUBANS: }} & 118.9 & $64 \cdot 2$ & & & 1.00 & & & & & & & \\
\hline & & $95 \times:$ & L IPSB : & 31.8 & 8.1 & I T B. & & $4 A=26$ & RGRBBS & & & & \\
\hline
\end{tabular}


TEARING STRENGTH, NO CUTOUT
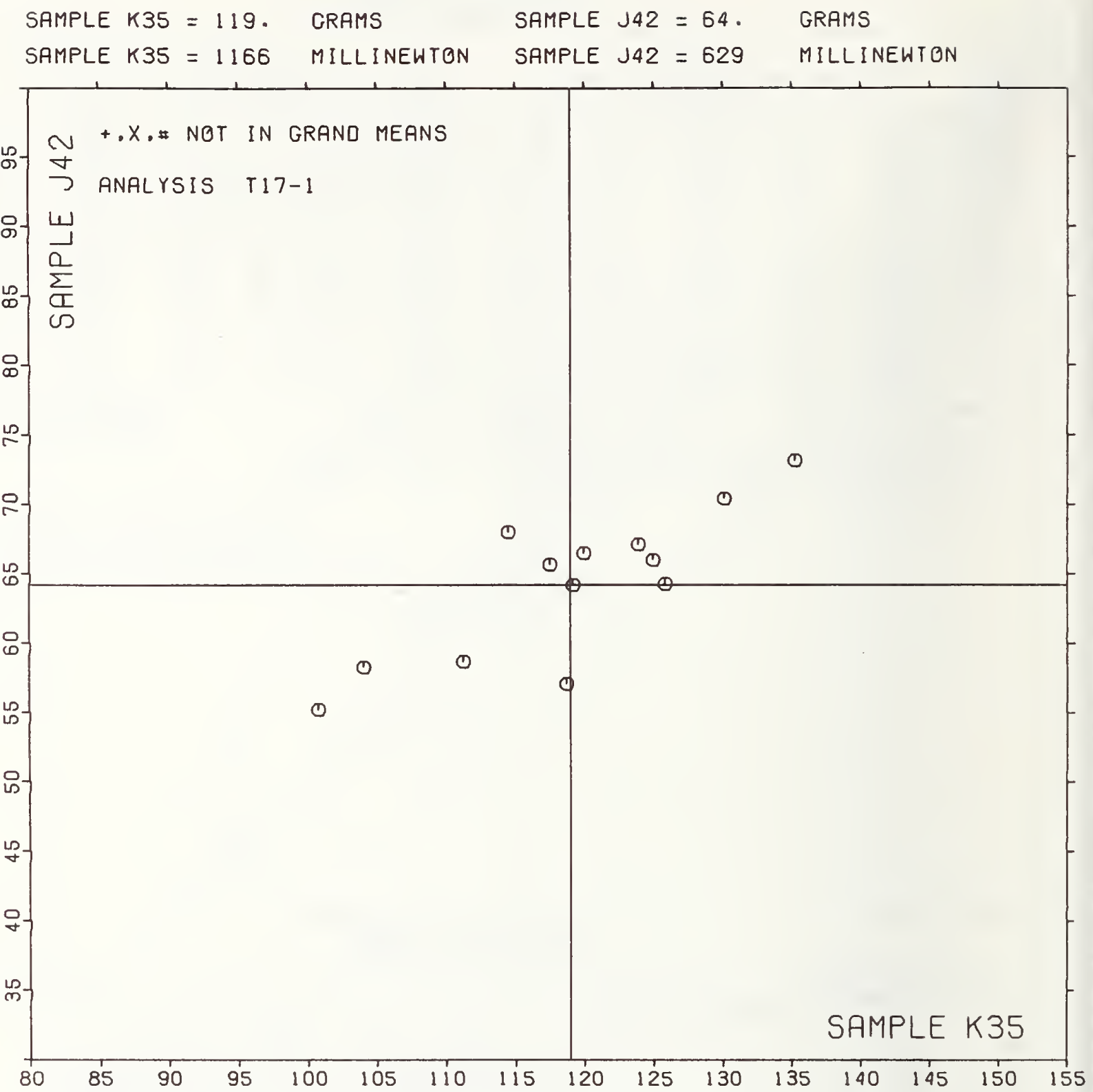
GRAMS

\begin{tabular}{|c|c|c|c|c|c|c|c|c|}
\hline$\Gamma$ & $T$ & 1 & $T$ & $T$ & $T$ & $T$ & $T$ & 1 \\
\hline 800 & 850 & 900 & 950 & 1050 & 1150 & 1250 & 1350 & 1450 \\
\hline
\end{tabular}


IBNSILE BREAKING STRBNGTH, KILJNBWTONS PER IETBR - PACKAGING PAPBR

IAPPI STANDAEDS T404 OS-76 $\triangle N D$ T494 OS-70, IBNSILE BRBATING STRBNGTH, PBNDULUN AND CRB TYPES

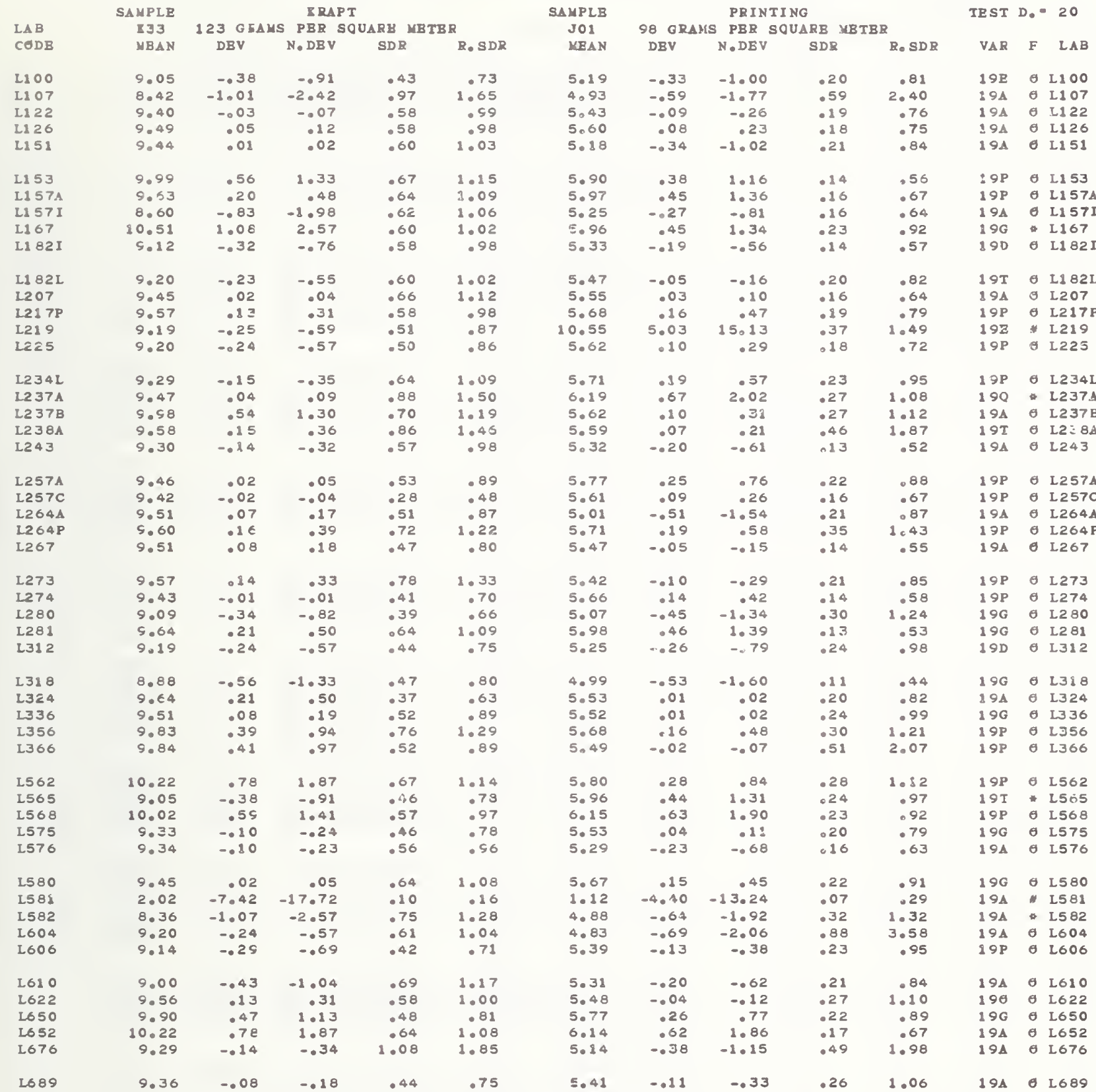

GR. MAN - 9.43 IILENBDTEN/M GRAND MELN - 5.52 KILGNBWTON/M

SD MBANS - 42 KILENBWTON/M

$\begin{aligned} \text { GRAND MELN } & 5.52 \text { RIL GNBWTON/M } \\ \text { SD OF MBANS } & .33 \text { KILGNBWTEN/Y }\end{aligned}$ AVERAGB SDR - .59 RILENBWTEN/M

GR. JEAN = $53.88 \mathrm{LB} / \mathrm{INCE}$

GRAND LEAN $-31.52 \mathrm{LB} / \mathrm{INCH}$

TBST DBTERMINATIENS - 20

49 LABS IN GRAND MEANS 25 BILONBWTEN/M

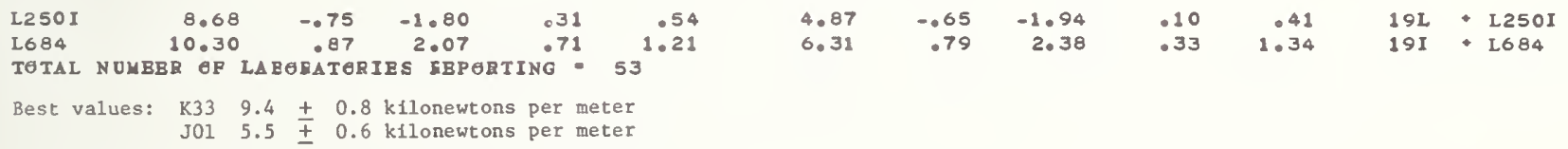

The following laboratorles were omitted from the

grand means because of extreme test results: 219.

Data from the following laboratories appear to be

off by a multiplicative factor: 581 . 
IENSILB BREAZING STRBNGTB, IILONBWTONS PBR IETBR - PACEAOING PAPBR

TAPPI STANDARDS T404 OS-76 AND I494 09-70, TBNSILB BRBARING STRBNGTB, PBNDULUM AND CRB IYPBS

\begin{tabular}{|c|c|c|c|c|c|c|c|c|c|c|c|c|c|}
\hline \multirow{3}{*}{$\begin{array}{l}\text { LAB } \\
\operatorname{CODB} \\
\text { L581 }\end{array}$} & \multirow{3}{*}{ F } & \multicolumn{2}{|c|}{ MBANS } & \multicolumn{2}{|c|}{ COORDINATBS } & \multirow{2}{*}{$\begin{array}{l}\text { AVG } \\
\text { R. SDR }\end{array}$} & \multirow[b]{2}{*}{ VAR } & \multirow{2}{*}{\multicolumn{2}{|c|}{ PROPB RTY _ - IBST }} & \multirow{2}{*}{\multicolumn{4}{|c|}{ INST RUMENT --- CONDITIONS }} \\
\hline & & $\mathbf{8 3 3}$ & $\mathrm{JOI}$ & MAJER & MINGR & & & & & & & & \\
\hline & & 2.02 & 1.12 & -8.59 & .76 & .23 & $19 \mathrm{~A}$ & TENSILB & STRBNOTE, & PACEAGING & PAPBR, & LOAD & $\operatorname{CBLL}(\operatorname{cBE})$ \\
\hline L582 & $*$ & 8.36 & 4.88 & $-1 \cdot 24$ & .11 & 1.30 & $19 A$ & TBNSIIE & STRBNGTB & PACKAGING & PAPBR, & $L \Theta \Delta D$ & CBLL (CRB) \\
\hline L807 & 0 & 8.42 & 4.93 & -1.17 & .11 & 2.02 & 194 & TBNGIIB & STRBNGTB & PACEAGING & PAPBR。 & LOAD & CBLL ( $\mathrm{CDB})$ \\
\hline L\$ 57 I & $\theta$ & 8.60 & 5.25 & -.93 & .27 & .85 & 194 & TBNSILB & STRBNOTB & PACKAGING & PAPBR. & LOAD & CBLL ( CRB ) \\
\hline L250I & - & 8.68 & 4.87 & -.99 & .009 & .47 & $19 \mathrm{~L}$ & TBNSILB & STRBNGTB, & PACEAGING & PAPBR. & CRB. & $20 \mathrm{C}, 65 \% \mathrm{RB}$ \\
\hline L38 8 & $\theta$ & 8.88 & 4.99 &. .76 & .011 & .62 & 190 & TBNSILB & STRBNGTB, & PACKAGING & PAPBR. & LOAD & CBLL (CRB) \\
\hline L100 & $\theta$ & 9.05 & 5.19 &. .50 & .005 & .77 & $19 \mathrm{~B}$ & TBNSILB & SIRBNGIB & PACKAOING & PAPBR. & LEAD & CBLL (CRB) \\
\hline L565 & 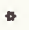 & 9.05 & 5.56 & .005 & .58 & .87 & $19 I$ & IBNSILB & SIRBNOTB, & PACKAGING & PAPBR. & PBNDU & DLUM TBSTBR \\
\hline L2 80 & $\boldsymbol{\theta}$ & 9.09 & 5.07 & $\because 54$ &. .16 & .95 & 190 & IBNS ILB & STRBNOTB. & PACKAOING & PAPBR. & LEAD & $\operatorname{CBLL}(\mathrm{CRB})$ \\
\hline L1 821 & $\theta$ & 9.12 & 5.33 & $\ldots 37$ & .04 & .78 & 190 & TBNSILB & STRBNOTB, & PACEAGING & PAPBR, & LOAD & CBLL ( $C R B)$ \\
\hline 2606 & $\theta$ & 9.14 & 5.39 & $\ldots 31$ & .07 &.$\$ 3$ & $19 P$ & IBNSILB & SIRBNOTB, & PACKAGING & PAPBR. & FBNDU & ULUM IBSTER \\
\hline 1289 & $n$ & 9.19 & 10.55 & 2.74 & 4.23 & 1.18 & $19 B$ & IBNSILB & STRBNOTB & PACKAOING & PAPBR. & LOAD & CBLL (CDB) \\
\hline L312 & $\theta$ & 9.19 & 5.25 & .035 & .007 & .86 & 190 & TBNSILB & STRBNGTB & PACTAGING & PAPBR. & LOAD & CBLL ( CDB ) \\
\hline 1604 & $\theta$ & 9.20 & $4 \cdot 83$ & -.59 &. .42 & $2 \cdot 31$ & 194 & TBNSILB & STRBNOIB, & PACKAGING & PAPBR. & LOAD & CBLL ( CDB ) \\
\hline L225 & $\theta$ & 9.20 & 5.62 & $\ldots 14$ & .22 & .79 & $19 P$ & IBNSILB & STRBNGTB, & PACXAGING & PAPBR. & PBNDO & DLOM TBSTBR \\
\hline L182L & $\theta$ & 9.20 & 5.47 &. .22 & .08 & .92 & 191 & TBNSILB & STRBNOTB, & PACKAGINO & PAPBR. & PBNDD & DLUM TBSTER \\
\hline $1234 \mathrm{~L}$ & $\theta$ & 9.29 & 5.72 & -.01 & .24 & 1.02 & $19 P$ & TBNSIIE & STRBNGTE. & PACKAGING & PAPBR. & PBNDU & ULUX IBSIBR \\
\hline 2676 & $\boldsymbol{\theta}$ & 9.29 & 5.14 &.- .34 &. .23 & 1.92 & 194 & TBNBILB & SIRBNOTB & PACKAGING & PAPBR. & LOAD & CBLL ( CRB ) \\
\hline $\mathrm{L} 243$ & $\theta$ & 9.30 & 5.32 & -.23 & .008 & .75 & 194 & T BNSILB & STRBNOTB. & PACKAGING & PAPBR. & LEAD & CBLL (CRE) \\
\hline L575 & $\theta$ & 9.33 & 5.55 &. .06 & .09 & .79 & 190 & TBNSILB & SIRBNGTB, & PACEAGING & PAPBR。 & LEAD & CBLL ( CRB ) \\
\hline L576 & $\theta$ & 9.34 & 5.29 &. .21 &. .13 & .80 & 194 & TBNSILB & STRBNGTB, & PACKAGINO & PAPBR, & LGAD & CBLL (CRE) \\
\hline 1689 & $\theta$ & 9.36 & 5.41 &. .12 & .0 .04 & .90 & 194 & IBNSILB & SIRBNGTB, & PACKAOING & PAPBR. & LOAD & CBLL ( CRB) \\
\hline L122 & $\theta$ & 9.40 & 5.43 & .0 .08 & .05 & .87 & 194 & TBNSI LB & STRBNGIB, & PACKAGING & PAPBR, & LEAD & CBLL ( CRB) \\
\hline $1257 \mathrm{C}$ & $\theta$ & 9.42 & 5.61 & .04 & .08 & .57 & $19 \mathrm{P}$ & IBNS I LB & SIRBNGTE, & PACKAGING & PAPBR, & PBNDU & ULUM TBSTBR \\
\hline 1274 & $\theta$ & 9.43 & 5.66 & .08 & .12 & .64 & $19 P$ & TBNSI LB & SIRBNGIB. & PACEAOINO & PAPBR, & PBNDU & ULUM IBSTER \\
\hline LI51 & $\theta$ & 9.44 & 6.18 & .019 &. .28 & .94 & 194 & TBNSILB & SIRBNGTB & PACKAGING & PAPBR, & LAAD & CBLL ( CRB) \\
\hline L207 & $\theta$ & 9.45 & 5.55 & .03 & .02 & .88 & 194 & TBNS ILB & SIRBNGIB & PACKAGING & PAPBR, & LOAD & CBLL ( $C D B)$ \\
\hline L580 & $\theta$ & 9.45 & 5.67 & .11 & .11 & .99 & 190 & TBNSILB & STRBNGTB & PACKAGING & PAPBR. & LOAD & CBLL (CRB) \\
\hline L257A & $\theta$ & 9.46 & 5.77 & .16 & .19 & .89 & $19 \mathrm{P}$ & TBN SILB & SIRBNGTB. & PACRAgING & PAPBR. & PBNDU & ULUN TBSTER \\
\hline $1237 A$ & - & 9.47 & 6.19 & .42 & .52 & 1.29 & 190 & IEN SILB & STRBNGIB, & PACRAGING & PAPBR, & PBNDO & OLOM TBSTER \\
\hline L267 & $\theta$ & 9.51 & 5.47 & .03 &. .09 & .68 & 194 & TBNSILB & STRBNGIB, & PACKAGING & PAPBR. & LOAD & CBLL (CRB) \\
\hline 1336 & $\theta$ & 9.51 & $5 \cdot 52$ & .07 & -.04 & .94 & 190 & TBNSILB & SIRBNGIB, & PACKAGING & PAPBR, & LOAD & CBLL ( CRB ) \\
\hline L622 & $\theta$ & 9.56 & 5.48 & .08 & $\ldots 11$ & 1.05 & 190 & TBNSILB & STRBNGIB, & PACRAOINO & PAPBR, & PBNDU & ULUM TBSTBR \\
\hline L2\& $7 P$ & $\sigma$ & 5.57 & 5.68 & .20 & .05 & .89 & $19 \mathrm{P}$ & TBNAILB & STRBNGTB, & PACKUGINO & PAPBR, & PENDU & ULUM IBSTBR \\
\hline 1273 & $\theta$ & 9.57 & 5.42 & .06 &. .16 & 1.09 & $19 \mathrm{P}$ & TBN SILB & 8TRBNGIB, & PACZAOINO & PAPBR, & PBNDO & ULUN IBSTBR \\
\hline L238A & $\theta$ & 9.58 & 5.59 & .16 &. .03 & 1.67 & $19 T$ & TBN SILB & BTRBNGTB, & PACEAOING & PAPBR. & PBNDO & ULUM TBSIBR \\
\hline $1264 P$ & $\theta$ & 9.60 & 5.71 & .24 & .06 & 1.32 & $19 P$ & TBN SI LB & SIRBNOTB, & PACEAGING & PAPBR. & PBNDU & ULUN TBSTBR \\
\hline L157A & $\theta$ & 9.63 & 5.97 & .43 & .25 & .88 & $19 \mathrm{P}$ & TENBILB & STRBNGTH. & PACEAGING & PAPBR, & PBNDU & ULUM TBSTBR \\
\hline L281 & $\theta$ & 5.64 & 5.58 & .44 & .25 & .81 & 190 & TBN SI LE & STRBNOTH, & PACKAGINO & PAPBR. & IOAD & CBLL ( CRB) \\
\hline$L 324$ & $\theta$ & 9.64 & 5.53 & .17 &.- .12 & .73 & 194 & TBN SI LB & STRBNGIB, & PACRAOINO & PAPBR, & LOAD & CBLL ( CRE) \\
\hline L356 & $\theta$ & 9.83 & 5.68 & .48 & -.10 & 1.25 & $19 P$ & TBNSILB & STRBNGTB, & PACEAGING & PAPBR, & PENDO & ULUM TBSTBR \\
\hline L366 & $\theta$ & 9.84 & 5.49 & $\cdot 32$ & -.26 & 1.48 & $19 \mathrm{P}$ & TBNSILB & STRBNGTB, & PACKAOING & PAPBR, & PBNDU & ULUM TBSTBR \\
\hline 1650 & $\theta$ & 9.90 & 5.77 & .53 & .007 & .85 & 190 & TBNSILB & SIRBNGTE, & PACKAOING & PAPBR, & LEAD & CBLL ( CRB ) \\
\hline L237B & $\theta$ & 9.98 & 5.62 & .50 & -.23 & 1.15 & 194 & TBN SILB & SIRBNOTH, & PACRAGING & PAPBR. & LOAD & CBLL ( $C R B$ ) \\
\hline$L 1 \leqslant 3$ & $\theta$ & $\$ .99$ & 5.90 & .68 &. .01 & .85 & $19 \mathrm{P}$ & IBNSILB & BIRBNOTB, & PACKAGING & PAPBR, & PBNDU & UZUM TBSTBR \\
\hline L568 & $\theta$ & 10.02 & 6.15 & .85 & .17 & .95 & $19 P$ & TBNSILB & SIRBNOTB, & PACKAGING & PAPBR, & PENDU & ULUY TBSTBR \\
\hline 1652 & $\boldsymbol{\theta}$ & 10.22 & 6.14 & 1.00 & .04 & .88 & 194 & TBN SILB & BTRBNOTB & PACKAOING & PAPBR, & LOAD & CBLL (CRB) \\
\hline L562 & $\theta$ & 10.22 & 5.80 & .80 & -.23 & 1.13 & $19 \mathrm{P}$ & TBNSILB & BI RBNOTH. & PACKAGING & PAPBR, & PBNDU & ULUM TBST \\
\hline 1684 & $\bullet$ & 10.30 & 6.31 & 1.17 & .13 & 1.27 & 191 & TBNSILB & STRBNGTB, & PACELGINO & PAPBR, & CRB. & 20C, $65 \% \mathrm{RH}$ \\
\hline$L 167$ & $*$ & 10.51 & 5.56 & 1.13 & -.27 & .97 & 190 & I BNSILB & SIRBNGTH. & PACKAGING & $P \triangle P B R$ & LOAD & CBLL (CRB) \\
\hline G & & 9.43 & 2 & & & 1.00 & & & & & & & \\
\hline & & $95 \%$ & SB & .26 & 52 & I IB & & 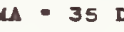 & BB 8 & & & & \\
\hline
\end{tabular}


TENSILE STRENGTH, PACKAGING PAPERS

SAMPLE K33 $=9.4 \quad$ KILONEWTON/M SAMPLE JOI $=5.5 \quad \mathrm{KILONEWION/M}$ SAMPLE K33 $=53.9 \quad$ LB/INCH SAMPLE JOI $=31.5 \quad \mathrm{LB} / I N C H$

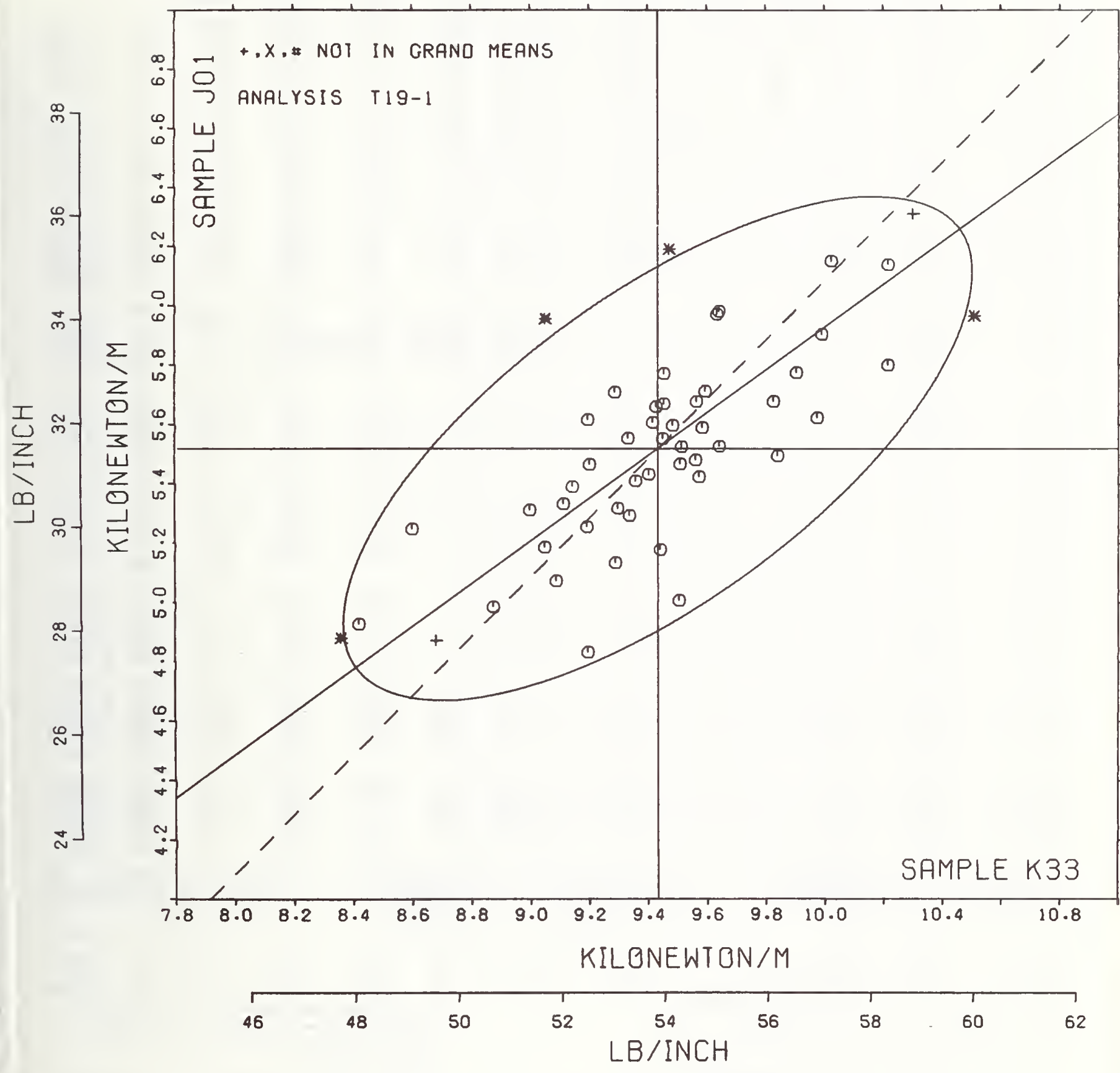


TBNBILE BREAEING OTRENGTB IILCNETTENB PBR IBTER

TAPPI STANDARD T494 OS-70. TBNSILB BRBAEING PROPBRTIBS OP PAPBR \& PAPBRBALRD (CONSTANT RATB OF BLGNGATIGN)

\begin{tabular}{|c|c|c|c|c|c|c|c|c|c|c|c|c|c|}
\hline \multirow{3}{*}{$\begin{array}{l}\text { LAB } \\
C A D B\end{array}$} & \multirow{3}{*}{$\begin{array}{c}\text { SAMPLB } \\
\text { JO6 } \\
\text { MBAN }\end{array}$} & \multicolumn{4}{|c|}{ PRINTINO } & \multicolumn{2}{|l|}{ SAMPLB } & \multicolumn{3}{|c|}{ PRINTING } & \multirow[t]{2}{*}{ IEST } & D. - & \multirow[t]{2}{*}{ - 20} \\
\hline & & 85 G FAM & 8 PBR 8 & OQUARB HE & & $J 71$ & 76 ORAM & 8 PBP & 8QUARE MBT & & & & \\
\hline & & DB V & N. DBV & GDR & R. SDR & MBAN & DBV & $N \cdot D B V$ & 8DR & R. SDR & $\mathbf{V} \boldsymbol{\Delta R}$ & $\mathbf{F}$ & $L_{\triangle} \mathbf{B}$ \\
\hline 1100 & 5.13 & -.18 & -1.08 & .17 & .74 & 3.63 & .0 .01 & -.08 & .12 & .75 & $20 B$ & $\boldsymbol{a}$ & 1200 \\
\hline L1 05 & 5.62 & .31 & 1.81 & .27 & 1.17 & 3.69 & .05 & .41 & .14 & .90 & 204 & 0 & L105 \\
\hline L122 & 5.24 & -.07 & .041 & .18 & .76 & 3.66 & .02 & .15 & .14 & .87 & 204 & 0 & 1122 \\
\hline $2124 \mathrm{C}$ & 5.27 &. .04 &. .22 & .23 & 1.00 & $3 \cdot 51$ &. .13 & -.98 & .21 & 1.36 & 204 & $\theta$ & L1 240 \\
\hline 1225 & 5.48 & .05 & .55 & .35 & 1.53 & 3.82 & .28 & 1.39 & .13 & .83 & $20 C$ & 0 & 1125 \\
\hline L131 & 5.44 & .13 & .73 & .17 & .71 & 3.78 & .14 & 1.11 & .13 & .82 & $20 B$ & $\boldsymbol{0}$ & 2131 \\
\hline L\&41 T & 5.22 & .05 & -.50 & .22 & .93 & 3.61 & -.03 & -.20 & .13 & .81 & 204 & 0 & $1141 \mathrm{I}$ \\
\hline 1243 & 5.87 & .56 & 3.28 & .21 & .89 & 4.01 & .37 & 2.85 & .12 & .74 & $2 O B$ & - & 1143 \\
\hline L148 & 5.50 & .55 & 3.44 & .22 & .95 & 3.87 & .24 & 1.82 & .18 & 1.16 & 204 & 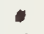 & 2148 \\
\hline 183 & 3.40 & .09 & .50 & .18 & .77 & 3.72 & .08 & .60 & .13 & .80 & 201 & $\theta$ & 2163 \\
\hline 2185 & 5.17 & .014 &. .81 & .37 & 1.59 & 3.44 & -.18 & -1.50 & .21 & 1.36 & $20 c$ & $\sigma$ & 2185 \\
\hline LI9OR & 5.26 & .005 & .30 & .26 & 1.13 & 3.65 & .01 & .10 & .14 & .91 & 204 & 0 & 21908 \\
\hline L194 & 5.11 & -.21 & -1.20 & .17 & .71 & 3.55 & -.09 & .070 & .13 & .85 & 204 & $\sigma$ & 2194 \\
\hline L22 $3 B$ & 5.49 & .18 & 1.05 & .12 & .52 & 3.73 & .09 & .73 & .14 & .87 & 201 & 0 & L223E \\
\hline 22260 & 5.37 & .06 & .35 & .46 & 2.00 & 3.62 & .001 & -011 & .29 & 1.85 & $20 \mathrm{C}$ & 0 & 12260 \\
\hline 1230 & 5.24 & .007 & -.41 & .16 & .70 & $3 \cdot 54$ &. .10 & .74 & .08 & .53 & 200 & $\sigma$ & L230 \\
\hline 1243 & 5.36 & .05 & .29 & .11 & .46 & 3.58 & -.05 & -.41 & .16 & 1.04 & 204 & $\sigma$ & 1243 \\
\hline L2ES & 5.44 & .13 & .77 & .17 & .74 & NO DATA & RBPORTE & D PER & EAMPLB J71 & & 201 & $\mathbf{M}$ & 2255 \\
\hline 1260 & 5.98 & .67 & 3.94 & .24 & 1.04 & 3.98 & .34 & 2.62 & .18 & 1.17 & 201 & $\bullet$ & $L 260$ \\
\hline 2268 & 5.49 & .18 & 1.04 & .28 & 1.19 & 3.86 & .22 & 1.72 & .18 & 1.13 & 204 & $\theta$ & $L 261$ \\
\hline L278 & 5.51 & .20 & 1.19 & .16 & .70 & 3.88 & .24 & 1.88 & .20 & 1.28 & 204 & $\sigma$ & 1278 \\
\hline L291 & 4.90 & -.41 & -2.39 & .19 & .81 & 3.59 & -.05 & -.38 & .17 & 1.05 & 204 & - & L291 \\
\hline 1309 & 5.16 & -.15 & -.87 & .25 & 1.07 & 3.65 & .01 & .09 & .12 & .76 & 208 & $\boldsymbol{\theta}$ & 1309 \\
\hline 2315 & 5.13 & $-.1 E$ & -1.08 & .23 & .98 & 3.43 &. .20 & -1.56 & .17 & 1.12 & 201 & $\sigma$ & 2315 \\
\hline 2318 & 4.98 & -.23 & -1.92 & .14 & .59 & 3.38 & -.26 & -1.98 & .10 & .63 & 200 & $\theta$ & 2318 \\
\hline 1325 & 5.32 & .00 & .03 & .20 & .87 & 3.62 & -.02 & -.17 & .14 & .88 & $20 B$ & $\boldsymbol{\theta}$ & 2325 \\
\hline$L 328$ & 5.09 &.$-<2$ & -1.29 & .19 & .82 & 3.59 &. .05 & -.37 & .16 & 1.03 & 204 & 0 & L328 \\
\hline L331 & 0.08 & .76 & 4.47 & .28 & 1.23 & 3.97 & .33 & 2.56 & .13 & .82 & 204 & 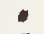 & L331 \\
\hline 2333 & 5.44 & .13 & .77 & .17 & .72 & 3.78 & .14 & 1.09 & .14 & .90 & 204 & 0 & 1.333 \\
\hline 2344 & 5.55 & .24 & 1.40 & .21 & .89 & 3.78 & .14 & 1.08 & .13 & .84 & 201 & $\sigma$ & 2344 \\
\hline 1352 & 5.52 & .20 & 1.20 & .41 & 1.74 & 3.79 & .16 & 1.20 & .16 & 1.04 & 204 & $\theta$ & $L 352$ \\
\hline 1356 & 5.42 & .11 & .64 & .15 & .63 & 3.64 & .01 & .05 & .14 & .98 & 204 & $\theta$ & 2356 \\
\hline 2360 & 5.40 & .08 & .53 & .21 & .90 & 3.69 & .06 & .43 & .10 & .65 & 208 & $\sigma$ & 2360 \\
\hline 2390 & 5.48 & .17 & .99 & .27 & 1.17 & 3. 76 & .12 & .92 & .12 & .79 & 204 & 0 & $L 390$ \\
\hline 1442 & 5.28 & -.03 & -.18 & .14 & .59 & 3.60 & $\ldots 04$ &. .28 & .10 & .62 & 200 & $\boldsymbol{\sigma}$ & 2442 \\
\hline 1557 & 5.05 & -.26 & -1.53 & .23 & .99 & 3.42 & -.22 & -1.68 & .16 & 1.01 & 204 & $\sigma$ & 1557 \\
\hline L558 & 1.13 & $-4.1 \mathrm{e}$ & $-24 \cdot 44$ & .06 & .27 & .75 & 2.89 & $-22 \cdot 19$ & .04 & .27 & 201 & - & L558 \\
\hline $\begin{array}{l}\text { L559 } \\
\text { L5634 }\end{array}$ & $\begin{array}{l}5.97 \\
5.51\end{array}$ & $\begin{array}{l}.66 \\
.20\end{array}$ & $\begin{array}{l}3.86 \\
1.18\end{array}$ & $\begin{array}{l}.14 \\
.29\end{array}$ & $\begin{array}{r}.62 \\
1.26\end{array}$ & $\begin{array}{l}4.09 \\
3.77\end{array}$ & $\begin{array}{r}.46 \\
.13\end{array}$ & $\begin{array}{l}3.51 \\
1.00\end{array}$ & $\begin{array}{l}.07 \\
.27\end{array}$ & $\begin{array}{r}.42 \\
1.70\end{array}$ & $\begin{array}{l}201 \\
201\end{array}$ & $\theta$ & $\begin{array}{l}1559 \\
25634\end{array}$ \\
\hline L567 & 5.22 & .009 & -.51 & .21 & .90 & 3.39 &. .25 & -1.93 & .16 & 1.04 & 201 & $\sigma$ & $L 567$ \\
\hline 1574 & 6.01 & .70 & 4.09 & .25 & 1.10 & 3.90 & .27 & 2.05 & .19 & 1.23 & 204 & 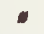 & 2574 \\
\hline 1575 & 5.31 & .00 & .02 & .24 & 1.01 & 3.64 & .00 & .04 & .19 & 1.19 & 200 & $\theta$ & 2575 \\
\hline L592 & 5.32 & .01 & .03 & .26 & 1.14 & 3.62 & -.01 & -.10 & .15 & .93 & 201 & 0 & L592 \\
\hline 2616 & 1.16 & -4.15 & -24.25 & .11 & .49 & .62 & -3.02 & -23.18 & .05 & .33 & 200 & 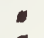 & 1616 \\
\hline 2618 & 5.38 & .06 & .38 & .49 & 2.12 & 3.53 & -.10 & .00 & .31 & 1.99 & 201 & 0 & 1618 \\
\hline
\end{tabular}

GR. MBAN - 5.31 IILONBTION/M SD LEANS. 17 KILONBUTON/M GR. NBAN $=17.912$ LB/15 MM
GPAND MRN - 3.64 EILONBVTON/Y BD OP MBANS - .13 IILONBTTON/Y .23 IILOKB TON/M AVBRAGE $8 D R$.

TEST DBTERMINATIONB - 20 36 IABS IN GRAND IRANS $.16 \mathrm{KILONE}$ TON $\mathrm{K}$

\begin{tabular}{|c|c|c|c|c|c|c|c|c|c|c|c|c|}
\hline 1239 & 5.33 & .02 & .13 & .28 & 1.20 & 3.61 & .003 &. .23 & .17 & 1.07 & $20 \mathrm{H}$ & - L1 39 \\
\hline L218 & 1.97 & -3.34 & -19.52 & .45 & 1.92 & 3.68 & .04 & .33 & .42 & 2.71 & $20 I$ & - L211 \\
\hline L250 I & 4.74 & -.57 & -3.32 & .14 & .59 & 3.23 &. .41 & -3.15 & 10 & 63 & 201 & - L2501 \\
\hline
\end{tabular}
.59 It 1 eupected thet the following loboratorlee recelved eaples whlch vere different froe the reet: $143,148,260$. 331 . 559.574 .

J71 $3.6 \pm 0.2$ kilonewtons per meter

Data from the following laboratories appear to be

off by a multiplicative factor: 558,616 .

For these Ix leboretoried the following epplie日:

\begin{tabular}{rrr} 
& 506 & 571 \\
SDMAN & 5.97 & 3.97 \\
\hline & .07 & .08
\end{tabular}


IBNAILB BREAITNG BTRBNOTH, IILONBWTENB PBR IRTER

TAPPI STANLARD T494 69-70, TINAILE BREAIING PROPBRTIBS OP PAPBR \& PAPBRBOARD (CONBTANT RATB OF BLENGATION)

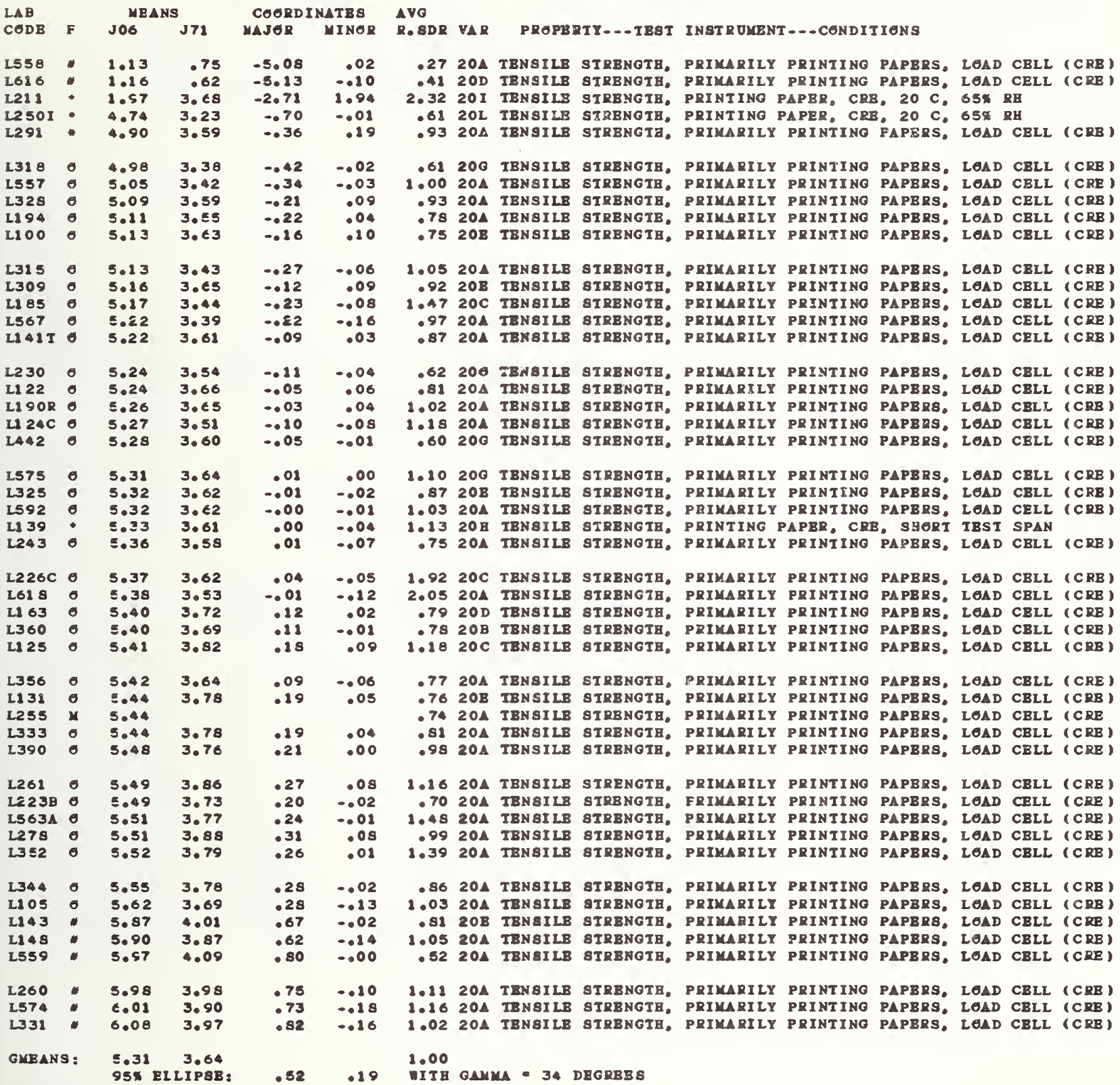


tensile stREnGTH, CRE TYPE

SAMPLE JO6 $=5.31$ KILONEWTON/M SAMPLE J71=3.64 KILONEWTON/M

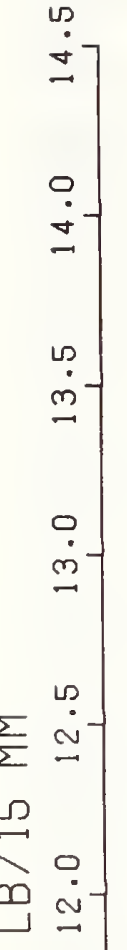

SAMPLE J06 $=17.91$ LB/15 MM SAMPLE J71 $=12.27$ LB/15 MM 
TAPPI STANDARD T404 OA-76, TBNAILE BREAIING GTRENOTB OP PAPBR $\triangle N D$ PAPBRBOARD (PBNDULOM-TYPB TBSTBR)

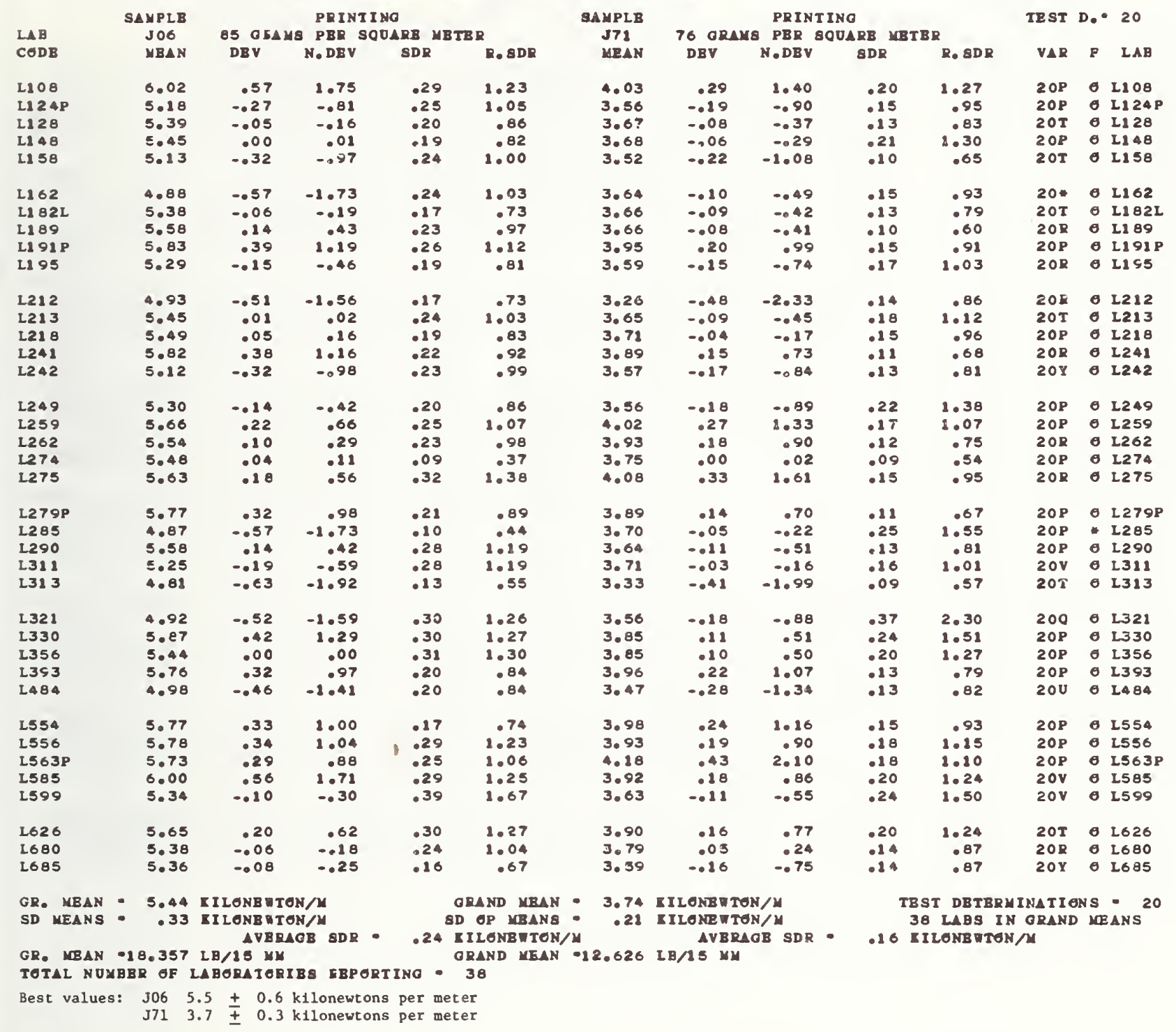




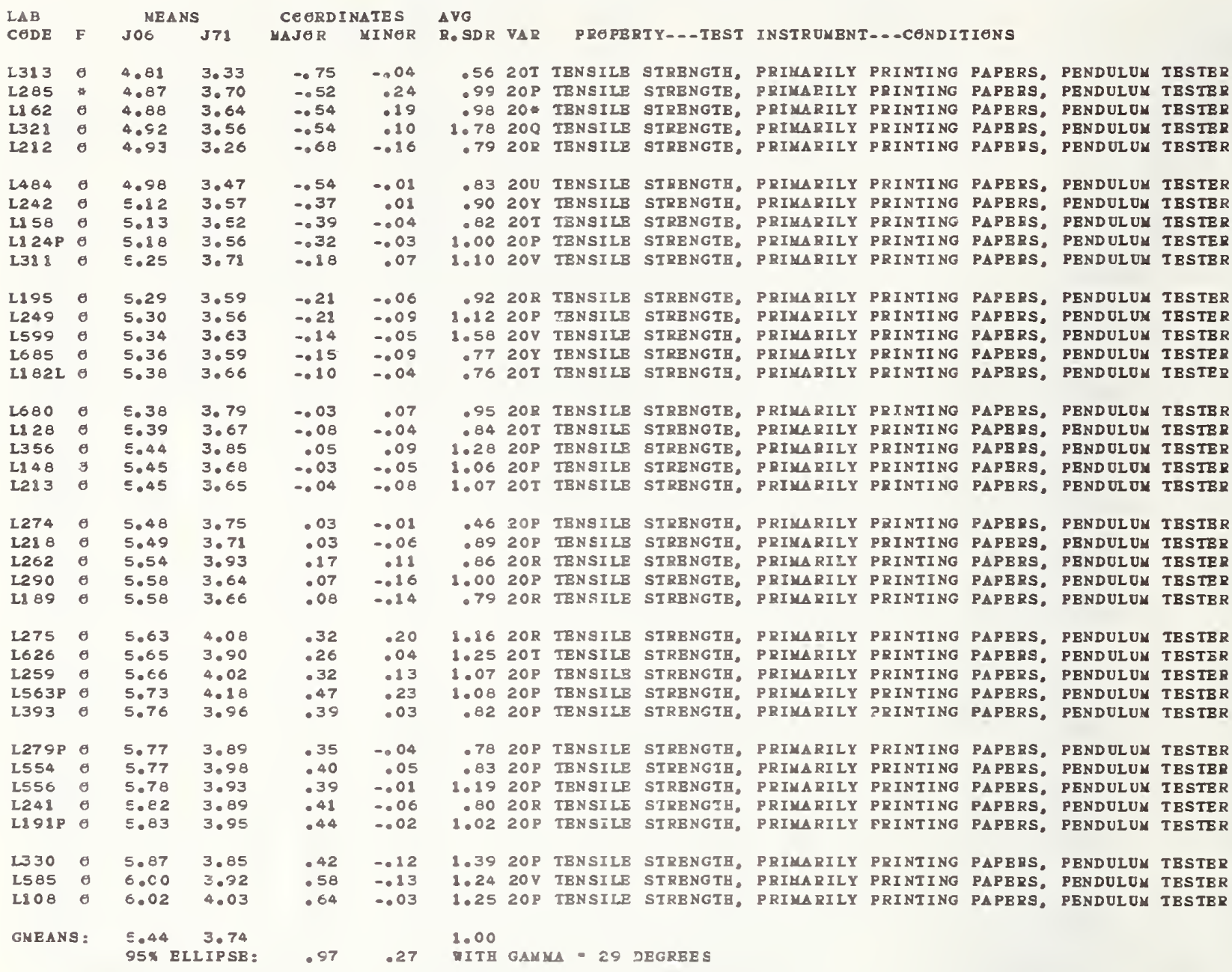


TENSILE STRENGTH, PENDULUM TYPE

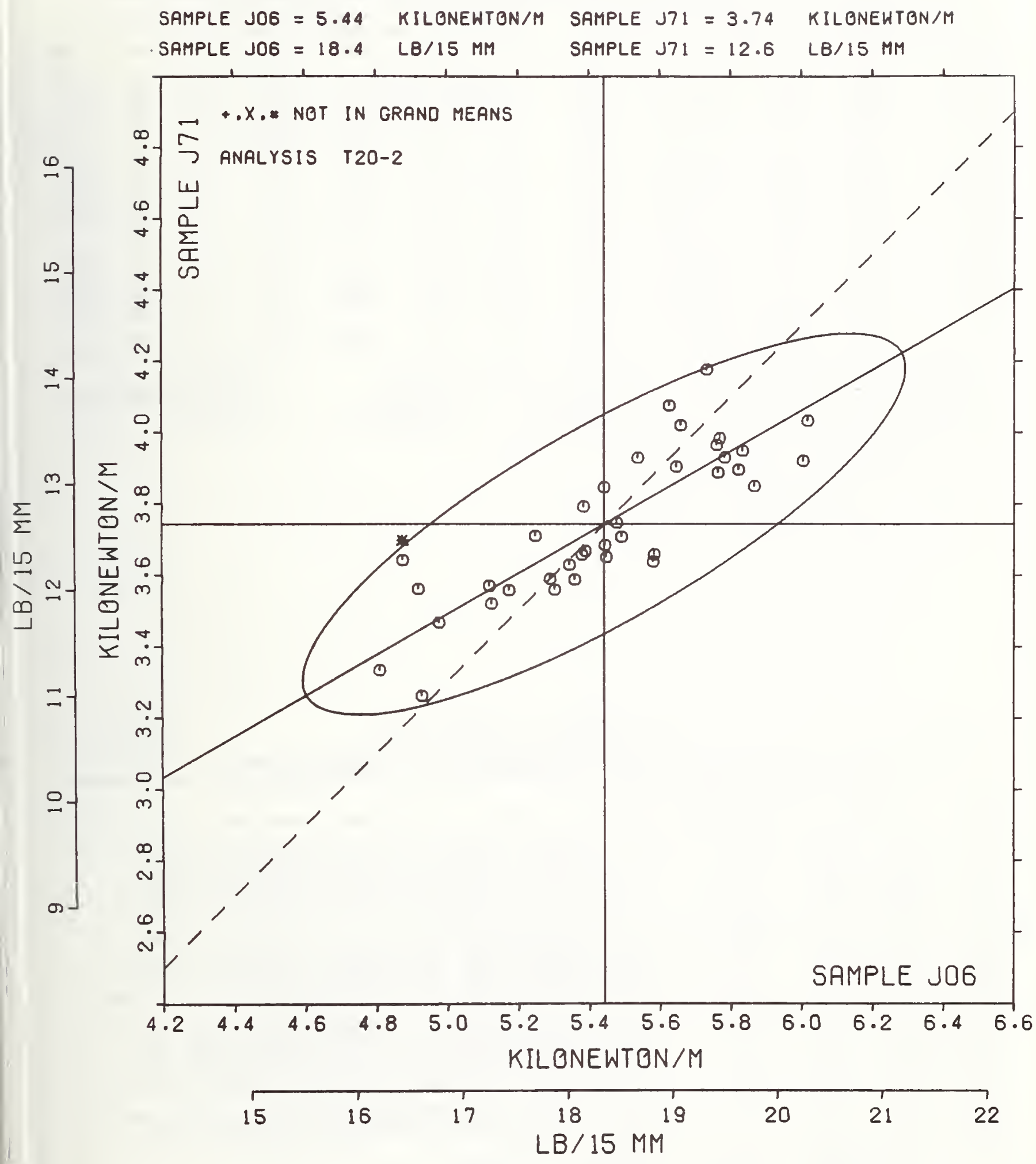


TBNSILB BNBROY $\triangle B$ BORTION, JEULES PBR SOUARB VOBTER - PACRAING PAPER

TAPPI STANDARD TA94 OS-70. TBNSILB BRBAIINO PREPBRTIBS OP PAPBR B PAPBRBOARD (CONSIANT RAIB OF BLONGATION)

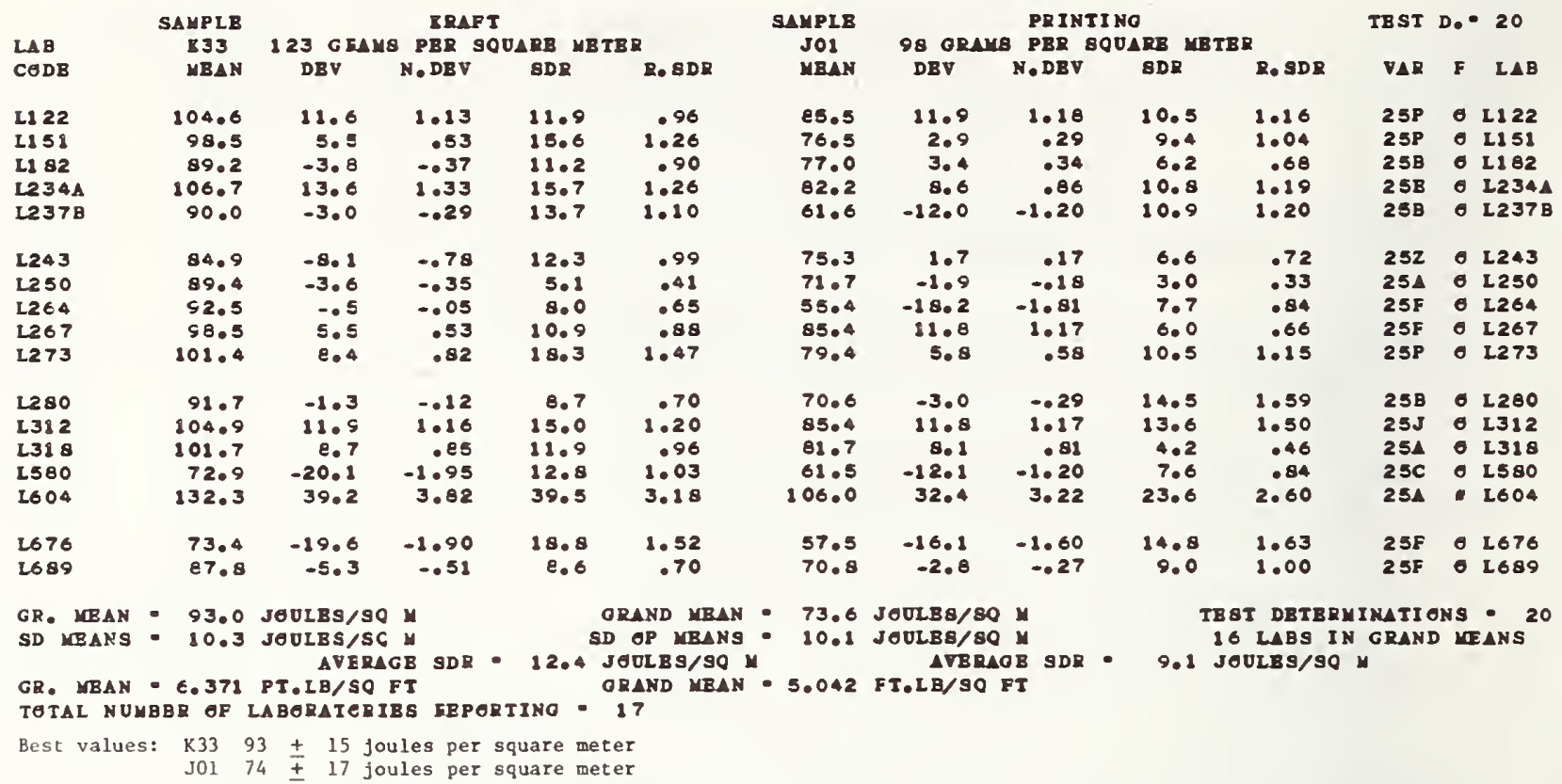

The following laboratorles were omitted from the

grand means because of extreme test results: 604.

\begin{tabular}{|c|c|c|c|c|c|c|c|c|c|c|c|c|}
\hline \multirow{2}{*}{$\begin{array}{l}\text { LAB } \\
C \in D E\end{array}$} & \multirow[b]{2}{*}{$\mathbf{F}$} & \multicolumn{2}{|c|}{ MBANS } & \multicolumn{2}{|c|}{ COERDINATBS } & \multirow{2}{*}{$\begin{array}{l}\text { AVG } \\
\text { R. SD R }\end{array}$} & \multirow[b]{2}{*}{ VAR } & \multirow{2}{*}{\multicolumn{4}{|c|}{ PROPBRTY---IBSI INGTRUMENT---CONDITIGNB }} & \\
\hline & & 133 & 301 & MAJOR & MINOR & & & & & & & \\
\hline L580 & $\theta$ & 72.9 & 61.5 & $-22 \cdot 8$ & $5 \cdot 3$ & .93 & $25 \mathrm{C}$ & TBNSILB & BNBRG T & $\triangle B S$. PACKAINO & PAPBR,LINE/L INB & JAvS \\
\hline$L 676$ & $\theta$ & 73.4 & 57.5 & $-25 \cdot 3$ & $2 \cdot 1$ & 1.57 & $25 F$ & IBN $B$ ILB & B NB ROY & $\triangle B 8 ., P \triangle C K A O I N O$ & PAPBR, LI NE/PLAT & $\mathbf{J \Delta} \mathbf{w}$ \\
\hline L243 & $\theta$ & 84.9 & $75 \cdot 3$ & -4.6 & 6.9 & .86 & 252 & TBNAILB & BNBRG Y & $\triangle 88$, РАCKAOINO & PAPBR, I INE/LINB & JAES \\
\hline L689 & $\theta$ & 87.8 & 70.8 & -5.7 & 1.7 & .85 & $25 P$ & IBN SI LB & BNBROY & $\triangle B B_{,}$, PACLAOINO & PAPER , I I NE/PLAT & JAES \\
\hline 1182 & $\theta$ & 89.2 & 77.0 & -.4 & 5.1 & .79 & 258 & TBN SILB & BNBRG Y & $\triangle B 8 . . P \triangle C K A O I N O$ & PAPBR, I INB/PLAT & $J \Delta=\mathbf{S}$ \\
\hline L2 50 & $\theta$ & 89.4 & 71.7 & -3.9 & 1.2 & .37 & 254 & TENSILB & B NB RG Y & $\triangle B B_{.}, P \triangle C Y A O I N O$ & PAPBR,FLAT/PLAT & JA $\mathbf{E} S$ \\
\hline L237B & $\theta$ & 90.0 & 61.6 & -10.5 & -6.5 & 1.15 & 25B & IBNSILB & B NB RG Y & АBS. PACKAINO & PAPBR,2-PIN STRA & $\triangle I N G A O B$ \\
\hline I 280 & $\theta$ & 91.7 & 70.6 & -3.0 & $-1 \cdot 2$ & 1.15 & $25 \mathrm{~B}$ & TBNB ILB & BNBROY & $\triangle B$ P. . PACKAOINO & PAPBR, II NE/PLAI & $J \Delta=S$ \\
\hline 1264 & $\theta$ & 92.5 & $\$ 5.4$ & $-13 \cdot 1$ & $-12 \cdot 7$ & .75 & $25 P$ & IBNSILB & BNBROY & $\triangle B S$. PACKAOINO & PAPBR, II NB/PLAI & $J \Delta E S$ \\
\hline L151 & $\theta$ & 98.5 & 76.5 & 6.0 & $-1 \cdot 7$ & 1.15 & $25 P$ & IBNBILB & BNBEOY & $\triangle B B_{.}$, PACRAOINO & PAPB R, P TTE RNBD & PLAT JAIS \\
\hline L267 & $\theta$ & 98.5 & 85.4 & 12.1 & 4.6 & .77 & $25 P$ & TBNSI LB & BNBROY & $\triangle B 8$. PACRAOINO & PAPBR, LINB/PLAT & $J \Delta \mathbb{S}$ \\
\hline 1273 & $\theta$ & 101.4 & 79.4 & 10.1 & $-1 \cdot 7$ & 1.31 & $25 P$ & TBN 8 I LB & BNBROY & $\triangle B 8$. . PACKAOINO & PAPBR, I INB/PLAT & $J \Delta \pm 9$ \\
\hline L318 & $\boldsymbol{\theta}$ & 101.7 & 81.7 & 11.9 & -.3 & .71 & 254 & IBNBI LB & BNBRGY & $\triangle B B_{.} . P \triangle C R A I N O$ & PAPBR, PLAT/PLAT & $J \Delta \mathbb{S}$ \\
\hline 1122 & $\boldsymbol{0}$ & 104.6 & 85.5 & 16.6 & .4 & 1.06 & $25 P$ & TBN 8 I LB & BNBRGY & $\triangle B 8_{.}$. PACYOINO & PAPBR, PAT TBRNBD & FLAT JAES \\
\hline 1312 & $\theta$ & 104.9 & 85.4 & 16.8 & .1 & 1.35 & $25 \mathrm{~J}$ & IBNSI LB & BNBROY & $\triangle B 8$. PАCKAOINO & PAPBR, L I NE/PLAT & JAES \\
\hline $1234 \mathrm{~A}$ & $\boldsymbol{\theta}$ & 106.7 & 82.2 & 15.8 & $-3 \cdot 3$ & 1.23 & $25 B$ & TBNAILB & BNBRG & $\triangle B S .$. PАCRAINO & PAPER,2-PIN STRA & IN $G \triangle G B$ \\
\hline L604 & $*$ & 132.3 & 106.0 & 50.7 & -4.1 & 2.89 & 254 & IBNSI LB & BNBROY & $\triangle B$ B. . PАCRAOINO & PAPBR,PLAT/PLAT & JAES \\
\hline GMEAN & & $\begin{array}{l}93.0 \\
95 \%\end{array}$ & $\begin{array}{l}73.6 \\
\text { IPSB : }\end{array}$ & $38 \cdot 3$ & 13.8 & $\begin{array}{l}1.00 \\
\text { II IB }\end{array}$ & $\boldsymbol{O}$ & 4 & DEGREES & & & - \\
\hline
\end{tabular}


ANAIYSIS T26-1 TABLB 1

TBNSILB BNBROY $\triangle B S E R T I O N$, JUULBS PBR SQUARE NGTBR - PRINTING PAPBR

TAPPI STANDARD TA94 OS-70. TBNSILB BRBAIINO PREPBRTIBS OP PAPBR B PAPBEBEARD (CENSIANI RATB OF BLONGATION)

\begin{tabular}{|c|c|c|c|c|c|c|c|c|c|c|c|c|c|}
\hline \multirow{3}{*}{$\begin{array}{l}\text { LAB } \\
C \otimes D B\end{array}$} & \multirow{3}{*}{$\begin{array}{l}\text { SAMPLB } \\
\text { JOG } \\
\text { MBAN }\end{array}$} & \multicolumn{4}{|c|}{ PRINTINO } & \multicolumn{2}{|l|}{ SAMPLE } & \multicolumn{3}{|c|}{ PRINTING } & \multirow[t]{2}{*}{ IBST } & \multirow{2}{*}{ D. - } & \multirow{2}{*}{ - 20} \\
\hline & & 85 OSAL & IS PBR & SQU $\triangle R B \quad M B$ & & J71 & 76 GRAMS & S PBR & SQDARB LGT & & & & \\
\hline & & DEV & $\mathrm{N} \cdot \mathrm{DBV}$ & SDR & R. SDR & $M B A N$ & DBV & N.DBV & SDR & $R, S D R$ & VAR & $\boldsymbol{F}$ & LAB \\
\hline 2100 & 54.7 & $-4 \cdot 2$ & -1.01 & 3.6 & .64 & 42.9 & $1 \cdot 8$ & .45 & 3.5 & .76 & 264 & $\theta$ & 2100 \\
\hline L12 21 & 34.8 & $-24 \cdot 1$ & -5.78 & $1 \cdot 2$ & .21 & 23.6 & $-17 \cdot 5$ & -4.43 & - 8 & .17 & 26D & $*$ & 2121 \\
\hline L1 22 & 62.7 & 3.8 & .90 & 5.8 & 1.04 & $44 \cdot 8$ & $3 \cdot 6$ & .92 & 4.6 & 1.01 & $26 L$ & $\theta$ & 1122 \\
\hline L1 39 & 58.4 & -.6 & -.13 & 7.9 & 1.43 & 37.4 & -3.7 &. .94 & 4.6 & 1.00 & $26 B$ & $\theta$ & I139 \\
\hline 1863 & 55.8 & .9 & .21 & 5.6 & 1.02 & 43.4 & $2 \cdot 2$ & .56 & $4 \cdot 0$ & .87 & $26 J$ & $\theta$ & 2163 \\
\hline I2 67 & 58.5 & -.4 & -.10 & 2.4 & .43 & 39.7 & $-1 \cdot 4$ & -.37 & 2.4 & .51 & $26 \mathrm{D}$ & $\theta$ & L167 \\
\hline L1 85 & 52.1 & -6.8 & $-1 \cdot \epsilon 4$ & 10.4 & 1.88 & $32 \cdot 8$ & $-8 \cdot 3$ & -2.10 & 6.7 & 1.46 & $26 C$ & $\theta$ & I185 \\
\hline L28 8 & 62.8 & 3.5 & .93 & 6.5 & 1.17 & 40.2 & -09 & -.23 & $\epsilon .6$ & 1.43 & $26 c$ & $\theta$ & I211 \\
\hline L250 & 53.2 & -5.8 & $-1 \cdot 38$ & $2 \cdot 5$ & .44 & 37.0 & $-4 \cdot 2$ & -1.06 & 3.4 & .73 & 264 & $\theta$ & L250 \\
\hline L255 & 60.2 & $1 \cdot 3$ & .31 & $3 \cdot 8$ & .69 & NE IATA & PBPGR TBD & FER & SAMPLB $J 71$ & & $26 P$ & $\mathbf{x}$ & L255 \\
\hline 1309 & 61.5 & $2 \cdot 6$ & .63 & 8.1 & 1.46 & 45.7 & $4 \cdot 6$ & 1.16 & 3.9 & .84 & $26 J$ & $\theta$ & 1309 \\
\hline L318 & 60.1 & $1 \cdot 2$ & .29 & 5.6 & 1.01 & 43.7 & $2 \cdot 6$ & .65 & $5 \cdot 2$ & 1.12 & 264 & $\theta$ & L318 \\
\hline 1356 & 69.3 & 6.4 & 1.53 & 5.1 & .91 & 43.6 & $2 \cdot 5$ & .63 & 5.6 & 1.21 & 264 & $\theta$ & 1356 \\
\hline 2393 & 58.2 & -.8 & -.18 & 4.2 & .76 & 38.8 & $-2 \cdot 4$ & -.60 & $4 \cdot 2$ & .91 & $26 \mathrm{~V}$ & 0 & L393 \\
\hline LA 42 & 63.7 & $4 \cdot 8$ & 1.16 & $4 \cdot 3$ & .78 & 47.0 & 5.9 & 1.48 & 3.4 & .73 & $26 \mathrm{~B}$ & $\theta$ & 2442 \\
\hline L567 & 47.7 & $-11 \cdot 2$ & -2.70 & $5 \cdot 2$ & .94 & $27 \cdot 1$ & $-14 \cdot 1$ & -3.55 & 4.9 & 1.06 & 264 & 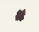 & L567 \\
\hline L575 & 53.9 & -5.0 & $-1 \cdot 20$ & 5.6 & 1.01 & 38.9 & $-2 \cdot 2$ & -.56 & 6.5 & 1.41 & 264 & $\sigma$ & 2575 \\
\hline I592 & 506.8 & 447.9 & 107.50 & 59.9 & 10.81 & 355.9 & 314.8 & 79.44 & 43.1 & 9.36 & $26 B$ & 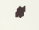 & L592 \\
\hline
\end{tabular}

GR. MBAN - $58.9 \mathrm{~J} E$ OLBS/SC M GRAND IBAN - $41.1 \mathrm{JOULBS/SO} \mathrm{M}$ SD MEANS = 4.2 JUULBS/SO

GR. MEAN - 4.036 PT. LB/EQ PT SD OF VEANS
4.0 JOULBA/SO M $\triangle V B R A G$ BDR
TEST DBTBRMINATIENS - 20

14 LABS IN ORAND MEANS $4.6 \mathrm{JOULBS} / \mathrm{SO}$ H

TETAL NUMBER OP LABERATERIBE SBPERTING - 18

Best values: J06 $59 \pm 6$ joules per square meter

J71 $41 \mp 6$ Joules per square meter

The following laboratories were omitted from the grand means because of extreme test results: 121, 567

Data from the following laboratories appear to be off by a multiplicative factor: 592 .

\begin{tabular}{|c|c|c|c|c|c|c|c|c|c|c|c|c|}
\hline LAB & & $\mathbf{M E}$ & NS & CEORDI & NATES & $A$ YG & & & & & & \\
\hline $\operatorname{CODB}$ & $\mathbf{F}$ & J06 & 371 & MAJER & MINOR & R. SDR & VAR & PROPB & RTY $=-$ I & BST INSTR UMBNT & -- CONDITIONS & \\
\hline L12 21 & $\bullet$ & 34.8 & 23.6 & -29.6 & 3.6 & .19 & $26 D$ & TBNSILB & BNBRGY & $\triangle B S \ldots$ PRINTINO & PAPBRS.2-PIN STRA & AIN GAGB \\
\hline L567 & 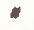 & 47.7 & 27.1 & $-17 \cdot 8$ & $-2 \cdot 6$ & 1.00 & 264 & TBNSI LB & BNBROY & $\triangle B S_{.}$, PRINTINO & PAPERS,PLAT/PLAT & JAWS \\
\hline L2 85 & $\theta$ & 52.1 & 32.8 & -10.7 & $-1 \cdot 4$ & 1.67 & $26 \mathrm{C}$ & IBNSI LB & BNBRGY & $\triangle B S ., P R I N T I N G$ & PAPBRS I INB/LINB & JAWS \\
\hline L250 & $\theta$ & 53.1 & 37.0 & $-7 \cdot 1$ & - 9 & .59 & 264 & IBNSILB & BNBROY & ABS. PRINTINO & PAPERS, PLAT/PLAT & JAWS \\
\hline L575 & $\theta$ & 53.9 & 38.9 & $-5 \cdot 2$ & $1 \cdot 8$ & 1.21 & 264 & TBNSILB & BNBRG Y & $\triangle B S ., P R I N T I N O$ & PAPER S, PLAT/PLAT & JA $\mathbf{A} S$ \\
\hline L100 & $\theta$ & 54.7 & 42.9 & $-1 \cdot 8$ & 4.2 & .70 & 264 & TBNSILE & BNBRGY & $\triangle B S .$, PRINTINO & PAPBRS, FLAT/PLAT & JAWS \\
\hline L393 & $\theta$ & 58.2 & 38.8 & $-2 \cdot 2$ & -1.2 & .84 & $26 \mathrm{~V}$ & TBNSI LE & BNBROY & $\triangle B 8$, , PRINTINO & PAPBRS, LINB/P LAT & JATS \\
\hline L139 & $\theta$ & 58.4 & 37.4 & -2.9 & $-2 \cdot 3$ & 1.22 & $26 \mathrm{~B}$ & TBNSILB & BNBRGY & $\triangle B S, \ldots$ P I NTINO & PAPBRS, 2 -PIN STRA & $\triangle I N$ GAGB \\
\hline L267 & $\theta$ & 58.5 & 39.7 & $-1 \cdot 3$ & -.8 & .47 & 26D & TBNSILB & BNBRGY & A BS. PR I NT I NO & PAPERS, 2-PIN STRA & AIN GAGB \\
\hline L1 63 & $\theta$ & 55.8 & 43.4 & $2 \cdot 1$ & 1.0 & .94 & $26 \mathrm{~J}$ & TBNSILB & BNB ROY & ABS. . PRI NTINO & PAPBRS, II NB/PLAT & $J_{\Delta} \equiv \mathbf{S}$ \\
\hline L318 & $\theta$ & 60.1 & 43.7 & 2.6 & $1 \cdot 1$ & 1.07 & 264 & TBNS I LB & BNBROY & $\triangle B S \ldots$ PR I NTINO & PAPBRS , PLAT/PLAT & JA WS \\
\hline L255 & $\mathbf{M}$ & 60.2 & & & & .69 & $26 P$ & TBNSI LB & BNBROY & $\triangle B S$, PRINTINO & PAPBRS, PATTBRNBD & PLAT JAES \\
\hline L309 & $\theta$ & 61.5 & 45.7 & $5 \cdot 1$ & 1.6 & 1.15 & $26 J$ & TBNSILB & BNBROY & $\triangle B S_{.}$. PRINTINO & PAPBRS, IINB/PLAT & JAWS \\
\hline L122 & $\theta$ & 62.7 & 44.8 & 5.2 & .1 & 1.03 & $26 L$ & TBNSILB & BNBROY & $\triangle B S ., P R I N T I N O$ & PAPERS P PITBRNBD & PLAT JAWS \\
\hline L.211 & $\theta$ & 62.8 & 40.2 & $2 \cdot 2$ & $-3 \cdot 3$ & $1 \cdot 30$ & $26 C$ & TBNSILB & BNBROY & $\triangle B S$. PRINTINO & PAPBRS , L INB/LINB & JADS \\
\hline 2442 & $\theta$ & 63.7 & 47.0 & $7 \cdot 5$ & 1.0 & .76 & $26 \mathrm{~B}$ & TBNSI LB & B NB RGY & $\triangle B S$. PR I NT INO & PAPBRS IINB/PLAT & JA $\mathbf{w S}$ \\
\hline 2356 & $\theta$ & 65.3 & 43.6 & $6 \cdot 3$ & -2.5 & 1.06 & $26 A$ & TENSILB & BNBROY & ABS . PR INIINO & PAPERS, FLAT/PLAT & $J \Delta=S$ \\
\hline L592 & $*$ & 506.8 & 355.9 & 542.1 & -76.0 & 10.08 & $26 \mathrm{H}$ & TBNSI LB & BNB RGY & ABS. . PR I NT INO & PAPBRS, 2-PIN STRA & AIN OAOB \\
\hline GMBAN & & 58.9 & TI.1 & & & 1.00 & & & & & & \\
\hline & & $95 \times$ & LLIPSB : & 15.6 & 5.9 & ET & 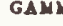 & $14 \cdot 43$ & DEOREES & & & \\
\hline
\end{tabular}




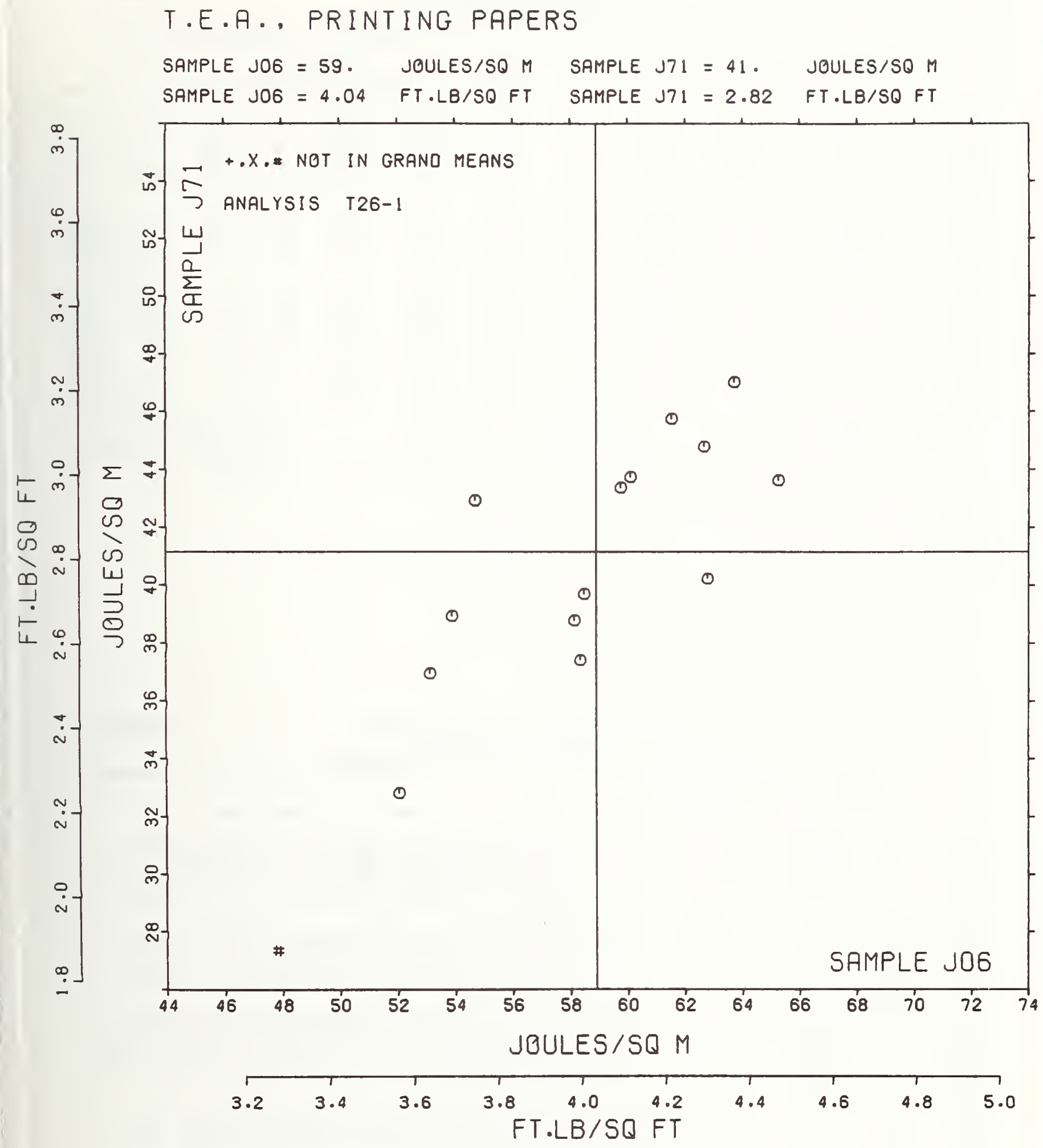


TAPPI SIANDARD T494 OS-70, TBNSILB BRBARINO PROPBRTIBS GP PAPBR \& PAPBRBOARD (CONSTANT RATB OP BLONGATION)

\begin{tabular}{|c|c|c|c|c|c|c|c|c|c|c|c|c|c|}
\hline \multirow{3}{*}{$\begin{array}{l}L A B \\
C O D B\end{array}$} & \multirow{3}{*}{$\begin{array}{c}\text { SAMPLB } \\
\text { B } 33 \\
\text { MBAN }\end{array}$} & \multicolumn{4}{|c|}{ 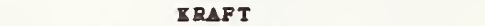 } & \multicolumn{2}{|l|}{ SAMPLB } & \multicolumn{3}{|c|}{ PRINTING } & \multirow[t]{2}{*}{ TB ST } & \multirow[t]{2}{*}{ D. - } & \multirow[t]{2}{*}{ - 20} \\
\hline & & 123 GEA & $S$ PBR 8 & $\triangle B B$ & & Jo1 & $98 \mathrm{GRA}$ & 5 PBR & SQOARB ME & & & & \\
\hline & & DBV & N.DBV & SDR & R. $S D R$ & MBAN & DBV & $N, D B V$ & SDR & R.SDR & VAR & $\mathbf{F}$ & $\mathbf{L} \mathbf{A B}$ \\
\hline 1100 & 1.53 &. .13 &. .59 & .09 & .57 & 2.07 & -.02 & .008 & .26 & .88 & $28 \Delta$ & 0 & 2100 \\
\hline 1122 & 1.77 & .10 & .45 & .10 & .64 & 2.26 & .16 & .62 & .19 & 1.05 & $28 P$ & $\theta$ & L122 \\
\hline 1126 & 1.57 &. .80 & -.044 & .13 & - 78 & 2.08 & -.01 & .005 & .15 & .86 & $28 C$ & 6 & L126 \\
\hline L158 & 8.94 & .27 & 1.19 & .20 & 1.20 & 2.28 & .18 & .71 & .23 & 1.30 & $28 \mathrm{~B}$ & 6 & L2 51 \\
\hline L1 82 & 1.55 &. .12 &.- .53 & .09 & .58 & 2.10 & .00 & .02 & .12 & .69 & $28 \mathrm{~B}$ & 0 & L182 \\
\hline 2243 & 1.45 & -.22 &. .56 & .11 & .69 & 2.02 & -.07 & -.28 & .13 & .71 & $2 B C$ & 6 & 1243 \\
\hline 1264 & 1.70 & .03 & .13 & .12 & .72 & 1.73 & -.36 & $-1 \cdot 38$ & .15 & .83 & $28 B$ & 6 & L264 \\
\hline 2267 & 1.60 & .007 & -.032 & .10 & .63 & 2.17 & .08 & .30 & .10 & .58 & $28 B$ & $\theta$ & 2267 \\
\hline L280 & 1.73 & .06 & .25 & .08 & .50 & 2.08 & .01 & .005 & .26 & 1.50 & $28 B$ & $\theta$ & L2 80 \\
\hline$L 382$ & 1.59 & .32 & 1.41 & .16 & .95 & 2.52 & .42 & 1.63 & .27 & 1.55 & $28 B$ & $\sigma$ & 2312 \\
\hline 2328 & 1.81 & .14 & .61 & .10 & .63 & 2.44 & .34 & 1.31 & .11 & .60 & 284 & $\theta$ & 2318 \\
\hline 1324 & 3.49 & -.18 & -.79 & .12 & .74 & 1.94 & -.16 & .061 & .14 & .82 & $28 P$ & $\sigma$ & 2324 \\
\hline 2336 & 1.71 & .04 & .18 & .10 & .60 & $2 \cdot 22$ & .12 & .47 & .20 & 1.12 & 281 & $\theta$ & 1336 \\
\hline 2580 & 1.28 & -.35 & $-1 \cdot 72$ & .18 & 1.12 & 1.56 & .053 & -2.03 & .12 & .70 & $28 c$ & $\theta$ & L5 80 \\
\hline 2581 & 1.50 &. .17 & .076 & .14 & .85 & 1.71 & .039 & -1.48 & .23 & 1.29 & 284 & 0 & L581 \\
\hline 2582 & 2.25 & .58 & 2.56 & .51 & 3.15 & 2.50 & .40 & 1.55 & .00 & .00 & 284 & $\theta$ & L5 82 \\
\hline 2676 & 1.64 & -.03 & -.13 & .45 & 2.75 & 2.09 & -.02 & .002 & .48 & 2.70 & $28 B$ & $\sigma$ & $L 676$ \\
\hline 2689 & 1.54 &. .12 &. .55 & .15 & .90 & 1.93 &. .26 & -.61 & .14 & .81 & $28 B$ & 6 & L689 \\
\hline
\end{tabular}

GR MEAN - 1.67 PBRCBNT GRAND MBAN - 2.10 PBRCBNT SD MEANS - 23 PBRCBNT SD OF MBANS - .26 PBRCBNT

SVERAGE SDR - .16 PBRCBNI
PDI

AVBRAGB SDR -

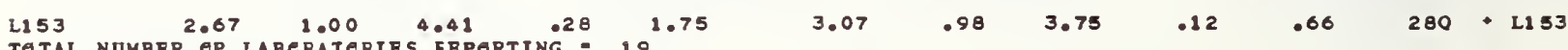

TBST DETBRYINATIENS - 20

18 LABS IN GRAND MBANS 18 PBRCBNT TOTAL NUMBER EP LABERATERIES FBPERTING - 19

Best values: $\mathrm{k} 331.7 \pm 0.3$ percent

j01 $2.1 \mp 0.4$ percent

REPORT NG. 578

TAFPI CELLABORATI VB REFBRBNCB PREORAY $\triangle N A L Y S I B$ I28-1 TABLB 2

DECEMBER 1978

ELGNGATION TO BRBAT. PBRCBNT - PACEGING PAPBR

TAPPI SIANDARD T494 OS-70, TBNSILB BRBAIIG PROPBRTIBS GF PAPER \& PAPBRBOARD (CONSTANT RATB OF ELGNGATIGN)

\begin{tabular}{|c|c|c|c|c|c|c|c|c|c|c|c|c|}
\hline \multirow{2}{*}{$\begin{array}{l}\text { LAB } \\
\text { CODE }\end{array}$} & \multirow[b]{2}{*}{$\mathbf{F}$} & \multicolumn{2}{|c|}{ MEANS } & \multicolumn{2}{|c|}{ CEGRDINATES } & \multicolumn{2}{|l|}{ AVO } & \multirow{2}{*}{\multicolumn{3}{|c|}{ FROPBRTY_-_IBST INSTRUMBNT---CONDITIONS }} & \multirow[b]{3}{*}{ JAMS } & \\
\hline & & $\mathbf{R 3 3}$ & Jol & MAJER & MNOR & R. $8 D R$ & VAR & & & & & \\
\hline L580 & $\theta$ & 1.28 & 1.56 & -.66 & -.04 & .91 & $28 \mathrm{C}$ & BLONGAT ION, PACIAOINO & $P \triangle P B R, L O A D$ & CBLL, LINB/LINB & & \\
\hline L243 & $\theta$ & 1.45 & 2.02 & -.20 & .12 & .70 & $28 \mathrm{C}$ & BLGNOATION, PACEAOING & $P \triangle P B R, L Q A D$ & CBLL, LINB/LINB & $J \boldsymbol{J} \boxplus \mathbf{S}$ & \\
\hline I324 & $\sigma$ & 1.49 & 1.94 & -.24 & .04 & .78 & $28 \mathrm{P}$ & BLONGATION, PACEAGING & PAPBR, LOAD & CBLL, PAT TBRKBD & FL $\mathbf{A I}$ & JAMS \\
\hline L581 & $\theta$ & 8.50 & 1.71 & -.41 & -.12 & 1.07 & 284 & BLONGATION, PACKAOING & PAPER, LGAD & CBLL, PLAT/FLAT & JA $\mathbf{w S}$ & \\
\hline 2100 & $\theta$ & 1.53 & 2.07 & .10 & .09 & .73 & 284 & BLONGATION, PACKAOING & $P \triangle P B R, L O A D$ & CBLL, PLAT/PLAT & 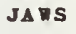 & \\
\hline 1689 & $\theta$ & 1.54 & 1.93 & -.20 & -.01 & .86 & $28 \mathrm{~B}$ & BLONGATION, PACEOINO & PAPBR, LGAD & CBLL, LINB/PLAT & JA $\mathbf{E S}$ & \\
\hline L1 e2 & $\theta$ & 1.55 & 2.10 & .007 & .09 & .64 & $28 B$ & BLONGATION, PACEOING & $P \triangle P B R, L O A D$ & CBLL,LINB/PLAT & JA $\mathbf{M S}$ & \\
\hline L1 26 & $\theta$ & 1.57 & 2.08 & .007 & .07 & .82 & $28 \mathrm{C}$ & BLONGATION, PACEAGING & $P \triangle P B R, L O A D$ & CBLL, IINB/LINB & JA $\mathbf{W S}$ & \\
\hline L267 & $\theta$ & 1.60 & 2.17 & .01 & .11 & .61 & $28 B$ & ELENOATION, PACZUGING & PAPBR, LQAD & CBLL, LINE/PLAT & JA S & \\
\hline 1676 & $\theta$ & 1.64 & 2.09 & -.02 & .02 & 2.73 & $28 \mathrm{~B}$ & BLONGATION, PACKAGINO & $P \triangle P B R, L O A D$ & $C B L L, L I N B / P L A T$ & JA $\mathbf{E S}$ & \\
\hline L264 & $\boldsymbol{\sigma}$ & 1.70 & 1.73 & -.26 & -.26 & .77 & $28 \mathrm{~B}$ & BLGNGATION, PACEAGING & PAPBR, LOAD & CBLL, LINB/PIAT & JA ws & \\
\hline 1336 & $\theta$ & 1.71 & 2.22 & .12 & .05 & .86 & 284 & ELONGATION, PACEAGING & PAPBR, LQAD & CBLL,PLAT/PLAI & JATS & \\
\hline 1280 & $\theta$ & 1.73 & 2.08 & .03 & .05 & 1.00 & $28 \mathrm{~B}$ & ELGNOATIEN, PACEATNO & PAPBR, LGAD & CBLL, IINB/PIAT & $J \Delta \oplus S$ & \\
\hline L12 2 & $\theta$ & 1.77 & 2.26 & .19 & .03 & .84 & $28 P$ & BLONGATIGN, PACKAOING & PAPBR, LOAD & CBLL, PATTBRNBD & FL $\Delta \mathbf{T}$ & JADS \\
\hline t38 8 & $\theta$ & 1.81 & 2.44 & .35 & .11 & .62 & $28 \Delta$ & BLONGATIEN, PACEAGING & $P \triangle P B R, L O A D$ & CBLL,PLAT/PLAT & JA $\mathbf{E S}$ & \\
\hline L151 & $\theta$ & 1.94 & 2.28 & .32 & -.09 & 1.25 & $28 B$ & ELGNGATIEN, PACZAOING & PAPBR, LOAD & CBLL, LINB/PLAT & JA $\mathbb{N S}$ & \\
\hline $\mathrm{L} 322$ & $\theta$ & 1.99 & 2.52 & .53 & .03 & 1.25 & $28 B$ & BLONOATION, PACEAOING & PAPBR, LOAD & CBLL, LINB/PLAT & JA $\mathbf{W} \mathbf{S}$ & \\
\hline LSB2 & $\theta$ & 2.25 & 2.50 & .68 & -.18 & $1 \cdot 57$ & 284 & BLONGATION, PACKAGING & PAPBR, LOAD & CBLL, PLAT/PLAT & $\mathbf{J \Delta} \mathbf{w}$ & \\
\hline $\mathbf{L 1 5 3}$ & - & 2.67 & 3.07 & 1.39 &. .14 & 1.20 & 280 & BLENGATION, PACRAGING & PAPBR, PBNDU & JLOM. PATTBRNBD & FLAT & JA $\mathbb{S}$ \\
\hline GMI & & $\begin{array}{l}1.67 \\
95 \times\end{array}$ & $\begin{array}{l}2.10 \\
\text { IP SB : }\end{array}$ & 91 & .29 & $\begin{array}{l}1.00 \\
\text { II I B }\end{array}$ & & $4=49$ & & & & \\
\hline
\end{tabular}


ELONGATION TO BREAK. PACKAGING PAPER

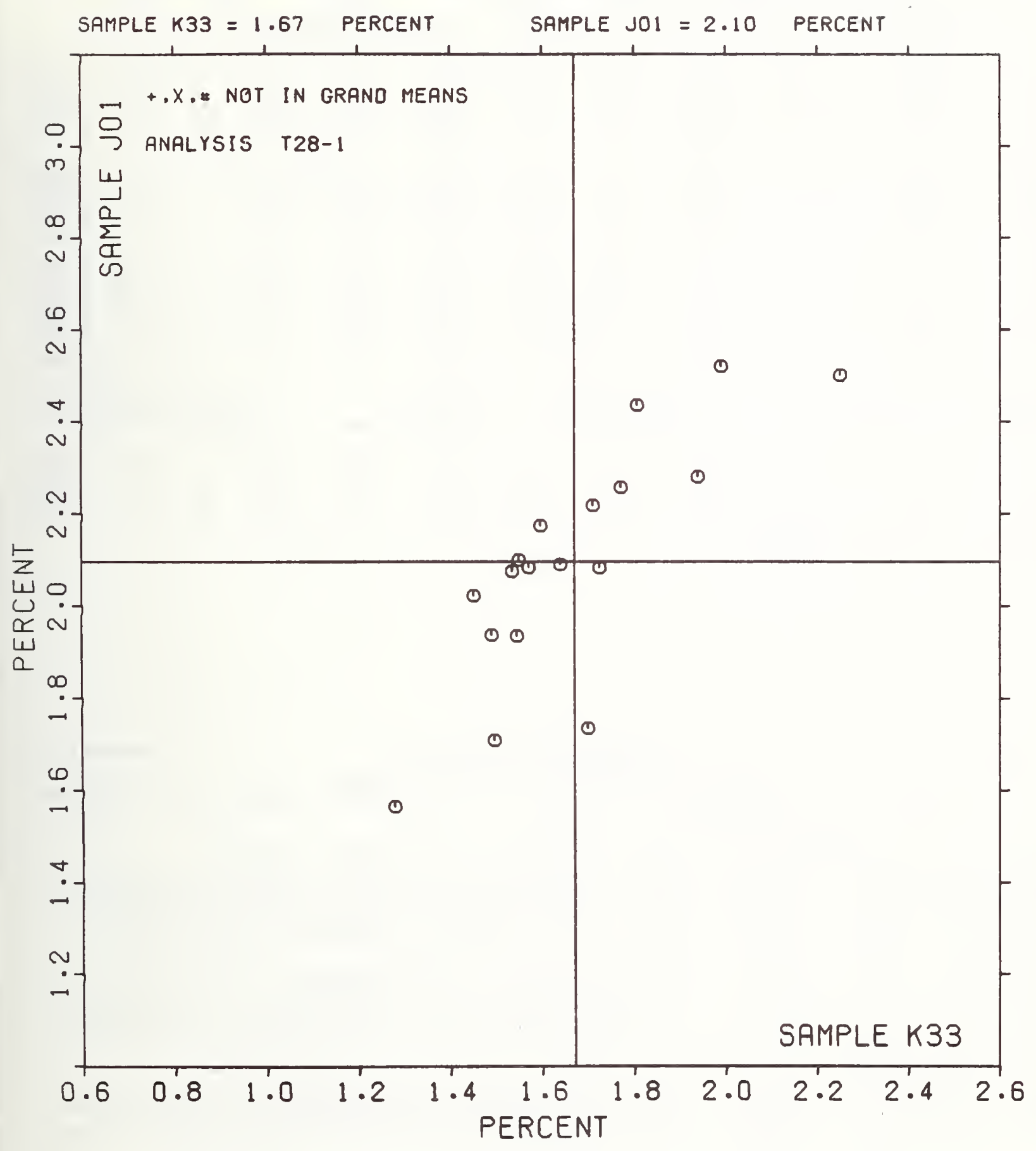


BIONGATION TO BPBAR PBRCBNT - PRINTING PAPBR

TAPPI STANLARD T494 OS-70, IENSILB BREAEING PROPBRTIBS GF PAPBR A PAPBRBARD (CONSTANT RATB GP BLENGATION)

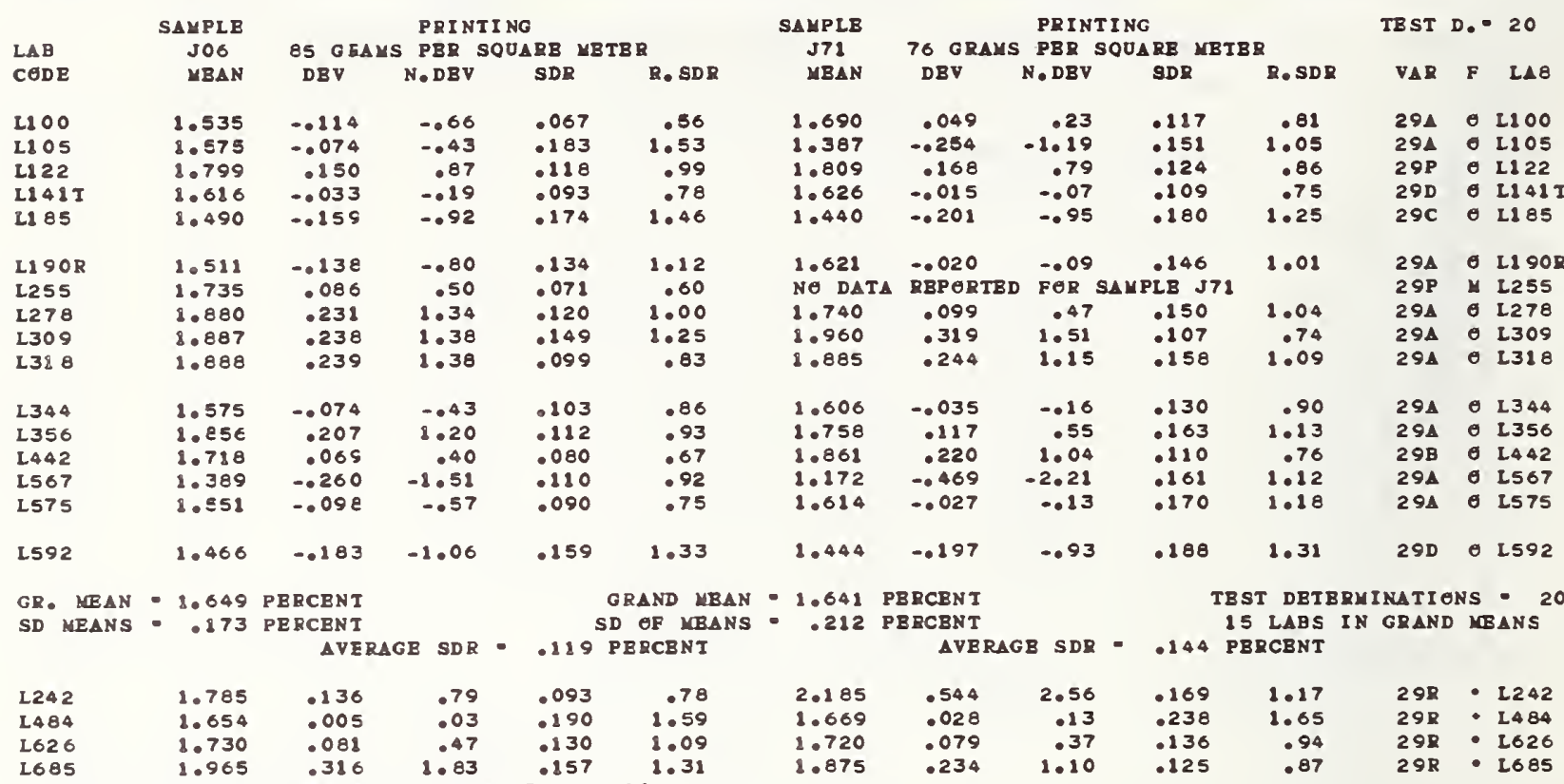

IOTAL NUYBER EF LABGRATEEIES REPERTING 20

Best values: J06 $1.65+0.23$ percent

$3711.64 \mp 0.31$ percent

RBPERT Ne. $57 \mathrm{~S}$

IAPPI COLLABORATIVB RBFBRENCB PROGRAN

DBCBMBER 1978 ANALYSIS I29-1 TABLB 2

BLONGATION TO BRBAT, PBRCBNI - PRINIING PAPBR

TAPPI STANIARD T494 OS-70, TBNBILB BREAIING PROPBRTIBS OP PAPBR B PAPBRBAAD (CONSTANT RATB OF ELENGATION)

\begin{tabular}{|c|c|c|c|c|c|c|c|c|c|c|c|c|}
\hline LAB & & $\mathbf{M B}$ & & COCRDI & NATEB & A VG & & & & & & \\
\hline CODE & $\mathbf{F}$ & J06 & 571 & MAJER & MINOR & R. SDR & $\mathbf{V} \mathbf{A R}$ & PROPBRTY---TEET II & SSTRUMBNT $---C$ & CONDIIIONS & & \\
\hline 1567 & $\theta$ & 1.389 & 1.172 &. .530 & .085 & 1.02 & 294 & BLENGATION, PRINTING & PAPERB, LOAD & CBLI,PLAT/PLAT & JA $\mathbf{W}$ & \\
\hline L592 & $\theta$ & 1.466 & 1.444 &. .268 & .022 & $1 \cdot 32$ & 290 & BLONGATION, PRINIING & PAPERB, LAAD & CBLL, 2-PIN STRA & $\mathbf{I N} \mathrm{G}$ & $\triangle G \mathbf{G}$ \\
\hline L185 & $\theta$ & 1.490 & 1.440 &. .256 & .001 & 1.36 & $29 \mathrm{C}$ & ELONGATION, PRINTING & PAPERS, LADD & CBLL, LINE/LINE & JAIS & \\
\hline L190R & $\theta$ & 1.511 & 1.621 &. .101 & .096 & 1.07 & $29 A$ & BLENGATION, PRINTING & PAPERS, LOAD & CELL,PLAT/PLAT & JANS & \\
\hline 2100 & $\theta$ & 1.535 & 1.690 & .032 & .120 & .68 & $29 \Delta$ & BL $O N G A T I O N$, PRINTING & PAPERS, LOAD & CBLI, PLAT/PLAT & JA WS & \\
\hline L575 & $\theta$ & 1.551 & 1.614 & .082 & .060 & .97 & 291 & BLENGATION, PRINTING & PAPERS, L $\triangle \triangle D$ & CELL, PLAT/PLAT & JA WS & \\
\hline L105 & $\theta$ & 1.575 & 1.387 &. .245 & .098 & 1.29 & $29 A$ & BL ONGATION, PRINIING & PAPBRS, LQAD & CBLI, PLAT/PLAT & 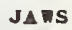 & \\
\hline L 344 & $\theta$ & 8.575 & 1.606 & .073 & .037 & .88 & $29 A$ & BLONGATION, PRINTING & PAPERS, LOAD & CELI, PLAT/PLAT & JA & \\
\hline $\mathrm{LI} 41 \mathrm{~T}$ & $\theta$ & 1.616 & 1.626 & .032 & .017 & .77 & 290 & BLONGATION, PRINTING & PAPERS, LOAD & CBLI, 2-PIN STR & $\mathbf{I I N} G$ & 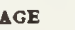 \\
\hline 2484 & - & 1.654 & 1.669 & .025 & .013 & 1.62 & $29 R$ & BLONGATION, PRINTING & PAPERS, PENDU & ULUX, PLAT/FLAT & JA & \\
\hline 2442 & $\theta$ & 1.718 & 1.861 & .216 & .082 & .72 & $29 B$ & BLONGATION, PRINIING & PAPERS, LOAD & CBLL, LINE/PLAT & JAMS & \\
\hline L626 & - & 1.730 & 2.720 & .112 & .015 & 1.02 & $29 R$ & BL ONGATION, PRINTING & PAPBRS, PENDU & ULUM, PLAT/FLAT & JA & \\
\hline 1255 & $\mathbf{y}$ & 1.735 & & & & .60 & $29 P$ & BLONGATION, PRINTING & PAPBRS, LOAD & CBLL, PATTBRNBD & FLAT & JAWS \\
\hline 1242 & - & 1.785 & 2.185 & .512 & .229 & .98 & $29 R$ & BLONGATION, PRINTING & PAPERS, PENDU & ULUY, PLAT/PLAT & JAWS & \\
\hline 2122 & $\theta$ & 1.799 & 1.809 & .225 &. .014 & .92 & $29 P$ & BLONGAIION, PRINTING & PAPBRS, LOAD & CBLL, PAT TERNBD & FI $\Delta \mathbf{T}$ & JATS \\
\hline L356 & $\theta$ & 1.856 & 1.758 & .220 & -.091 & 1.03 & 294 & ELONGATION, PRINIING & PAPERS, LOAD & CELL, FLAT/PLAT & JAWS & \\
\hline 1278 & $\theta$ & 8.880 & 1.740 & .220 & .120 & 1.02 & 294 & ELONGATION, PRINTING & PAPBRS, L QAD & CBLL, PLAT/PLAT & JAmS & \\
\hline 1309 & $\theta$ & 1.E87 & 1.960 & .398 & .010 & .99 & 294 & BL ONGATION, PRINTING & PAPERS, LOAD & CELI,PLAT/PLAT & JAWS & \\
\hline L31 8 & $\theta$ & 1.888 & 8.885 & .339 & .0037 & .96 & 294 & BLONGATION, PRINIING & PAPBRS, LOAD & CBLI, FLAI/PLAI & JA WS & \\
\hline 1685 & $\cdot$ & 1.565 & 1.875 & .379 & -.104 & 1.09 & $29 R$ & ELONGATION, PRINTING & PAPERS, PENDU & ULUX, FLAT/PLAT & JAWS & \\
\hline GMB A & & $\begin{array}{c}\text { 1. } 649 \\
95 \%\end{array}$ & $\begin{array}{l}\text { 8.641 } \\
\text { LLIPSE: }\end{array}$ & .754 & 213 & $\begin{array}{l}1.00 \\
\text { DITB }\end{array}$ & GAM & $V_{A}=51$ DEGRBES & & & - & \\
\hline
\end{tabular}


ELONGATION TO BREAK. PRINTING PAPER

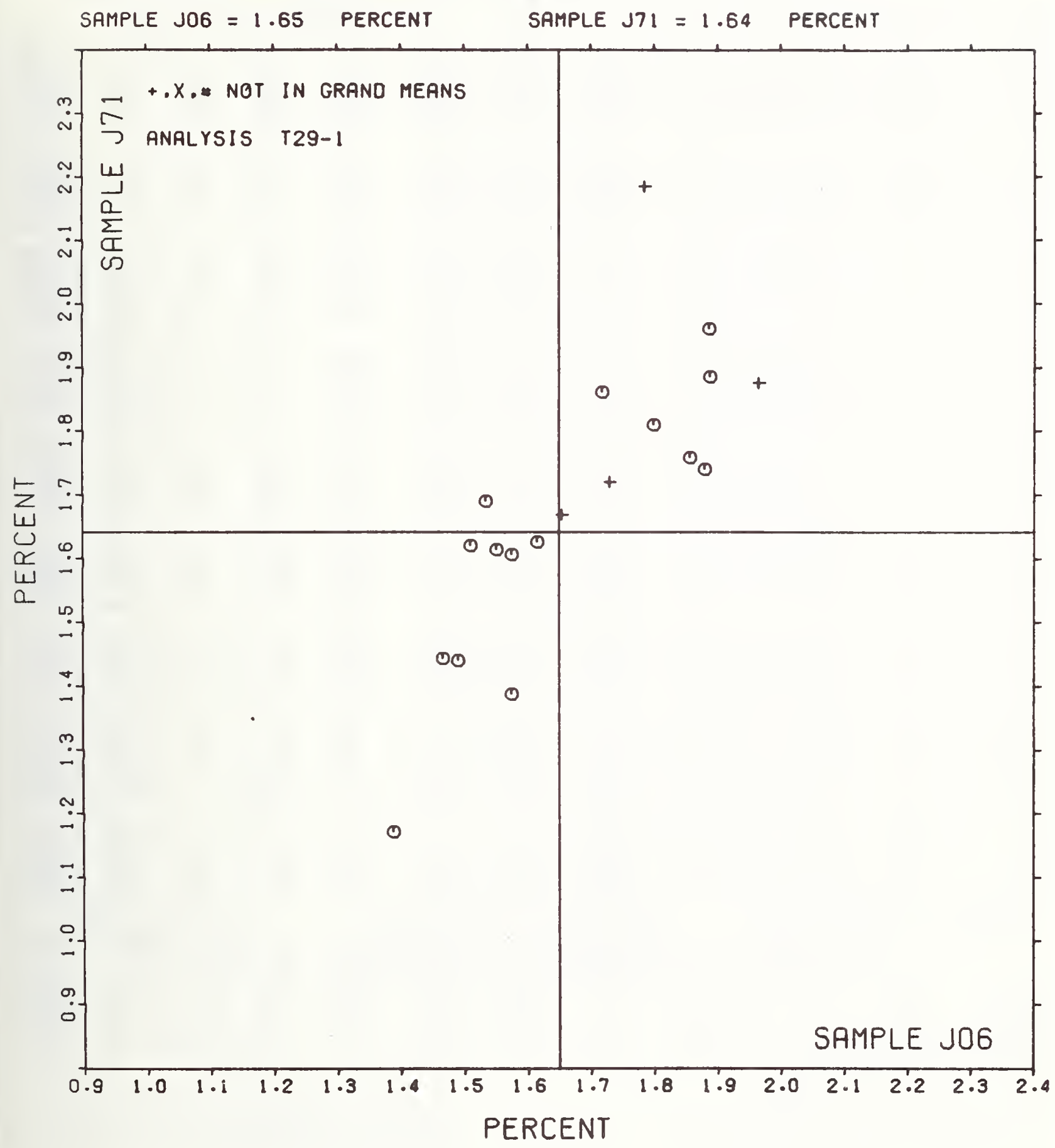


TAPPI STANDARD TS11 80.69

\begin{tabular}{|c|c|c|c|c|c|c|c|c|c|c|c|c|c|}
\hline & SAMPLE & & PRINT & IINO & & SAMPLE & & PRINT & IING & & TBST & D. - & 15 \\
\hline LAB & $\mathbf{J} \mathbf{3 0}$ & B5 GFAMS & PBR $\mathbf{8}$ & SQOARB MBZ & TBR & 532 & B6 GRAI & MS PBR S & SQUARB ME & IET B R & & & \\
\hline CODB & MBAN & DB V & N.DEV & SDR & $R_{0}$ SD R & MBAN & DEV & $\mathrm{N}, \mathrm{DBV}$ & $\operatorname{SDR}$ & $R \cdot S D R$ & VAR & $\mathbf{F}$ & LAB \\
\hline L1 OOM & 16.1 & -5.4 & -.81 & 2.2 & .30 & 63.4 & $-7 \cdot 4$ & -.40 & 7.8 & .45 & $30 \mathrm{M}$ & $\sigma$ & L100X \\
\hline LIOON & 15.8 & $-5 \cdot 6$ & -.85 & 1.9 & .25 & 65.3 & $-5 \cdot 4$ & -30 & 7.1 & .41 & $30 \mathrm{~N}$ & - & $2100 \mathrm{~N}$ \\
\hline 2105 & $21 \cdot 6$ & .2 & .03 & 6.2 & .85 & 69.3 & $-1 \cdot 4$ & .08 & 24.0 & 1.40 & 304 & $\sigma$ & 1105 \\
\hline L128 & 2.9 & -18.5 & -2.78 & .6 & .08 & 27.9 & $-42 \cdot 9$ & -2.34 & 9.2 & .53 & 304 & $*$ & L121 \\
\hline L12E & 24.3 & 2.8 & .43 & 10.5 & 1.44 & 94.0 & 23.2 & 1.27 & 19.9 & 1.16 & $30 \mathrm{x}$ & $\theta$ & L122 \\
\hline L1 24 & 21.7 & .3 & .05 & 4.9 & .68 & $72 \cdot 3$ & 1.6 & .09 & 11.0 & .64 & $30 N$ & $\theta$ & L1 24 \\
\hline L250 & 19.2 & $-2 \cdot 2$ & -.33 & 4.3 & . 59 & 60.9 & -9.8 & -.54 & 16.6 & .97 & $30 \mathrm{M}$ & $\sigma$ & L1 50 \\
\hline 2158 & 14.4 & -7.0 & -1.06 & 4.5 & .62 & 41.9 & $-28,8$ & -1.58 & 13.0 & .76 & $30 \mathrm{~N}$ & $\theta$ & L158 \\
\hline 1162 & 19.5 & -2.0 & -.29 & 4.3 & .59 & 65.2 & -5.6 & -.30 & 18.4 & 1.07 & $30 y$ & $\theta$ & 1162 \\
\hline L2 63 & 28.5 & -3.0 &.- .44 & 3.1 & .43 & 46.9 & $-23 \cdot 8$ & -1.30 & 13.9 & .81 & $30 \mathrm{~N}$ & $\theta$ & L163 \\
\hline L1 82M & 29.5 & 8.0 & 1.21 & 21.2 & 2.90 & 87.2 & 16.4 & .90 & 24.7 & 1.44 & $30 x$ & $\theta$ & L1 B2M \\
\hline L1 85 & 21.1 & $\ldots 3$ & .04 & 6.7 & .91 & 84.3 & 13.5 & .74 & 15.3 & .89 & $30 N$ & $\boldsymbol{\sigma}$ & 2185 \\
\hline $1190 \mathrm{C}$ & 32.2 & 10.7 & 1.61 & 9.6 & 2.31 & 108.0 & 37.2 & 2.03 & 33.0 & 1.93 & $30 \mathrm{~N}$ & $\sigma$ & LI $90 \mathrm{C}$ \\
\hline L21 2 & 21.0 & $\ldots 4$ & -.06 & 3.8 & .52 & 70.4 & -.4 & $\ldots 02$ & 14.5 & .84 & $30 \mathrm{x}$ & 0 & I212 \\
\hline L223F & 18.0 & -3.4 & -.51 & 4.8 & .66 & 76.4 & 5.6 & .31 & 13.5 & .79 & $30 \mathrm{x}$ & $\theta$ & L223F \\
\hline L230 & 17.5 & -3.5 & -.58 & 6.5 & .89 & 62.1 & $-8 \cdot 7$ & -48 & 13.5 & .79 & $30 N$ & $\theta$ & L230 \\
\hline L231 & 36.7 & 15.2 & 2.25 & 7.0 & .96 & 114.9 & 44.1 & 2.41 & 31.5 & 1.84 & $30 x$ & 6 & L231 \\
\hline L232 & 32.7 & 11.3 & 1.70 & 5.9 & .81 & 93.1 & $22 \cdot 3$ & 1.22 & 12.4 & .72 & $30 N$ & $\boldsymbol{\sigma}$ & L232 \\
\hline L236 & 16.1 & -5.4 & -081 & 2.5 & .34 & 63.2 & $-7 \cdot 6$ & $\because 41$ & 17.0 & .99 & $30 \mathrm{~N}$ & - & L236 \\
\hline L238A & 13.1 & $-8 \cdot 3$ & $-1 \cdot 25$ & 2.1 & .29 & 51.4 & -19.4 & -1.06 & 9.6 & .56 & $30 N$ & $\boldsymbol{\sigma}$ & L238A \\
\hline L238B & 15.2 & -6.2 &. .94 & 3.5 & .47 & 67.4 & -3.4 & $\ldots 18$ & 13.9 & .81 & 300 & o & $2238 B$ \\
\hline 1243 & $27 \cdot 5$ & 6.0 & .91 & 13.3 & 1.82 & 79.4 & 8.6 & .47 & 20.8 & 1.21 & 300 & $\theta$ & L243 \\
\hline 1254 & 81.7 & -5.8 & $-1 \cdot 47$ & 3.6 & .50 & 36.1 & -34.6 & $-1 \cdot 89$ & 18.1 & 1.06 & $30 x$ & $\theta$ & 1254 \\
\hline 1262 & 15.9 & -5.6 & -.84 & 3.2 & .43 & $61 \cdot 8$ & -9.0 & $\ldots 49$ & 14.4 & .84 & $30 N$ & $\boldsymbol{\theta}$ & 2262 \\
\hline 1274 & 34.3 & 12.5 & 1.94 & 19.0 & 2.60 & 100.3 & 29.6 & 1.61 & 16.6 & .97 & $30 N$ & $\theta$ & 1274 \\
\hline 1275 & 16.7 & $-4 \cdot e$ & -.71 & 3.6 & .50 & 84.5 & 13.7 & .75 & 17.4 & 1.01 & $30 N$ & $\theta$ & L275 \\
\hline 1278 & 11.8 & -5.6 & $-1 \cdot 45$ & 2.1 & .29 & $35 \cdot 2$ & -35.6 & -1.94 & 10.7 & .62 & $30 \mathrm{C}$ & $\theta$ & 1278 \\
\hline L279 & 17.5 & -3.5 & -.58 & 5.5 & .75 & 62.7 & -8.1 & -.44 & 19.7 & 1.15 & $30 \mathrm{~N}$ & $\theta$ & 1279 \\
\hline L2 $85 \mathrm{~A}$ & 20.5 & $-1 \cdot 0$ & -.14 & 4.3 & .59 & 76.9 & 6.2 & .34 & 14.8 & .86 & $30 \mathrm{~N}$ & $\theta$ & L $285 \mathrm{~A}$ \\
\hline L285B & 19.9 & $-1 \cdot 5$ & -.22 & 10.4 & 1.42 & 84.5 & 13.8 & .75 & $24 \cdot 8$ & 1.45 & $30 \mathrm{~N}$ & $\theta$ & L2 $85 B$ \\
\hline L259 & 18.7 & -2.7 & -.40 & 5.0 & .69 & 58.5 & $-12 \cdot 2$ & .067 & 19.2 & 1.12 & $30 \mathrm{~N}$ & $\theta$ & 1299 \\
\hline L320 & 18.1 & -3.4 & -.50 & 17.3 & 2.37 & 53.5 & $-17 \cdot 2$ & .94 & 17.8 & 1.04 & $30 \mathrm{~N}$ & $\theta$ & 1320 \\
\hline L326N & 16.3 & $-5 \cdot 1$ &. .77 & 11.1 & 1.52 & $52 \cdot 3$ & -18.4 & -1.01 & 17.7 & 1.03 & $30 \mathrm{~N}$ & $\theta$ & I326N \\
\hline 1339 & 15.3 & -6.1 & -.92 & 15.7 & 2.15 & 47.6 & $-23 \cdot 2$ & -1.27 & 12.2 & .71 & $30 N$ & $\theta$ & 2339 \\
\hline L366A & 25.2 & 3.8 & .57 & 20.7 & 2.84 & 78.3 & 7.5 & .41 & 17.7 & 1.03 & $30 \mathrm{~N}$ & $\theta$ & L366A \\
\hline$L 388$ & 41.0 & 19.6 & 2.95 & 31.1 & 4.26 & 74.9 & 4.1 & .22 & 10.2 & .60 & $30 \mathrm{~N}$ & $\mathbf{x}$ & 2388 \\
\hline 1390 & 20.4 & -1.0 & -.15 & E. 5 & .89 & 80.3 & 9.5 & .52 & 18.3 & 1.07 & $30 N$ & 6 & 1390 \\
\hline L393 & 32.5 & 11.0 & 1.66 & 14.0 & 1.91 & 62.3 & -8.4 & -.46 & 13.2 & .77 & $30 x$ & - & 1393 \\
\hline L396N & 28.9 & $7 \cdot 5$ & 1.13 & 19.7 & 2.70 & 55.2 & -15.6 & -.85 & 8.4 & .49 & $30 \mathrm{~N}$ & . & L396 M \\
\hline L565 & 28.5 & 7.1 & 1.07 & 13.9 & 1.90 & 94.7 & 23.9 & 1.30 & 25.2 & 1.47 & $30 N$ & $\theta$ & L565 \\
\hline$L 567$ & 29.5 & 8.0 & 1.21 & 8.7 & 1.19 & 82.1 & 11.3 & .62 & 24.1 & 1.40 & $30 \mathrm{~N}$ & $\theta$ & L567 \\
\hline L589 & 16.7 & $-4 \cdot e$ & -.71 & 3.1 & .43 & 70.3 & -.4 & .02 & 12.9 & .75 & $30 \mathrm{~N}$ & 0 & 1589 \\
\hline L559 & 30.9 & 9.5 & 1.43 & 7.2 & .98 & 70.2 & -.6 & .03 & 17.5 & 1.02 & $30 \mathrm{C}$ & o 1 & 1599 \\
\hline 1622 & 45.8 & 24.4 & 3.67 & 23.4 & 3.20 & $131 \cdot 8$ & 61.0 & 3.33 & 44.2 & 2.58 & $30 \mathrm{M}$ & $\mathbf{x}$ & L622 \\
\hline 2670 & 18.7 & -2.7 & -.40 & 2.4 & .33 & 88.1 & 17.3 & .94 & 15.4 & .90 & $30 \mathrm{~N}$ & ø & 1670 \\
\hline GR. MBAN & 21.4 & DOUBLE PELI & DS & & GRAND MBAN - & 70.8 & DOUBLB $F$ & OLDS & & TEST DETER & RXINATI Ө1 & NS & - 15 \\
\hline SD MEANS & 6.6 & DOUBLE PEII & DS & & SD OP MBANS & 18.3 & DOUBLB $P$ & OLDS & & 42 LABS I & IN GRAND & $\operatorname{LO}$ & ANS \\
\hline & & $\triangle V E R A G I$ & B SDR & 7.31 & DOUBLB FOLDS & 8 & AV BR & $\triangle O B$ SDR & 17.1 & DUUBLB PO & OLDS & & \\
\hline L182S & 19.0 & $-2 \cdot 4$ & -.36 & 4.7 & .64 & 144.1 & 73.3 & 4.00 & 26.9 & 1.57 & $30 \mathrm{~s}$ & - 1 & L182S \\
\hline L190D & 18.6 & $-2 \cdot 8$ & -.42 & 5.5 & .75 & 156.4 & 85.6 & 4.68 & 48.0 & 2.80 & 305 & - 1 & 11900 \\
\hline L280 & 13.2 & $-8 \cdot 2$ & $-1 \cdot 24$ & 3.7 & .50 & $61 \cdot 3$ & -9.4 & $\because 52$ & 12.8 & .74 & $30 x$ & - 1 & L280 \\
\hline $1326 \mathrm{~S}$ & 20.0 & -1.4 & -.21 & 25.3 & 3.47 & 127.1 & 56.4 & 3.08 & 76.3 & 4.45 & 305 & - 1 & $2326 \mathrm{~S}$ \\
\hline 3065 & & & & & & & & & & & & & \\
\hline
\end{tabular}

TOTAL NUMBER OF LABORATCRIBS FBPERIING - 50

J32 72 double folds

The following laboratorles were omitted from the

grand means because of extreme test results: 121.
The ISo (International Standarde frganizetion) is propoeing that MIT lolding endurance be reported as the logarithe (to the bese to) of the double fold inetead of the double fold ag In the paet.

Pleaee page 43 or this report for a demonetration of this proponal. 
FOLDINO ENDUPACB (MIT, DOURE FOLDS

TAPFI ITANDARD TSII OU-69

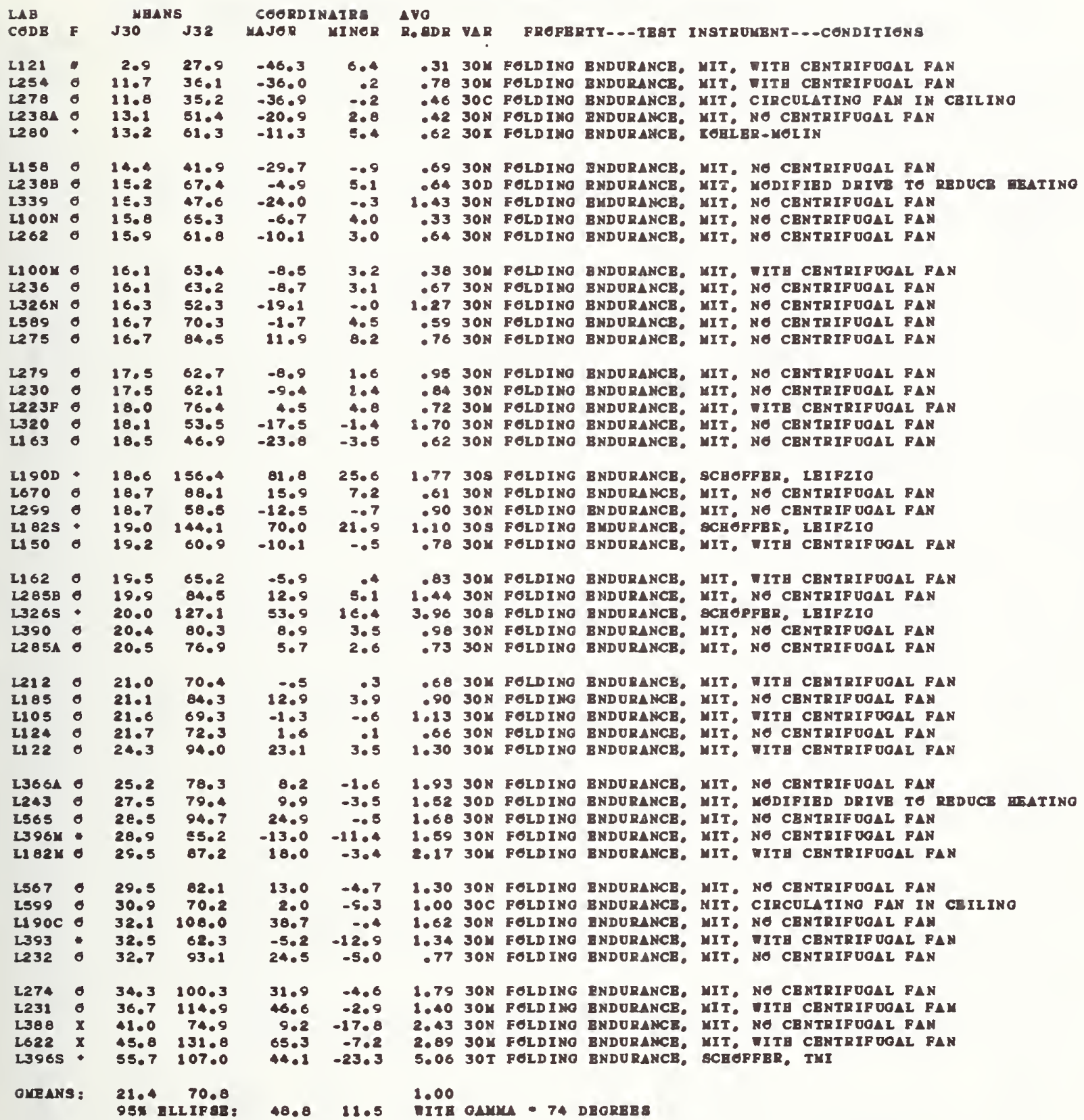


FOLDING ENDURANCE (MIT)

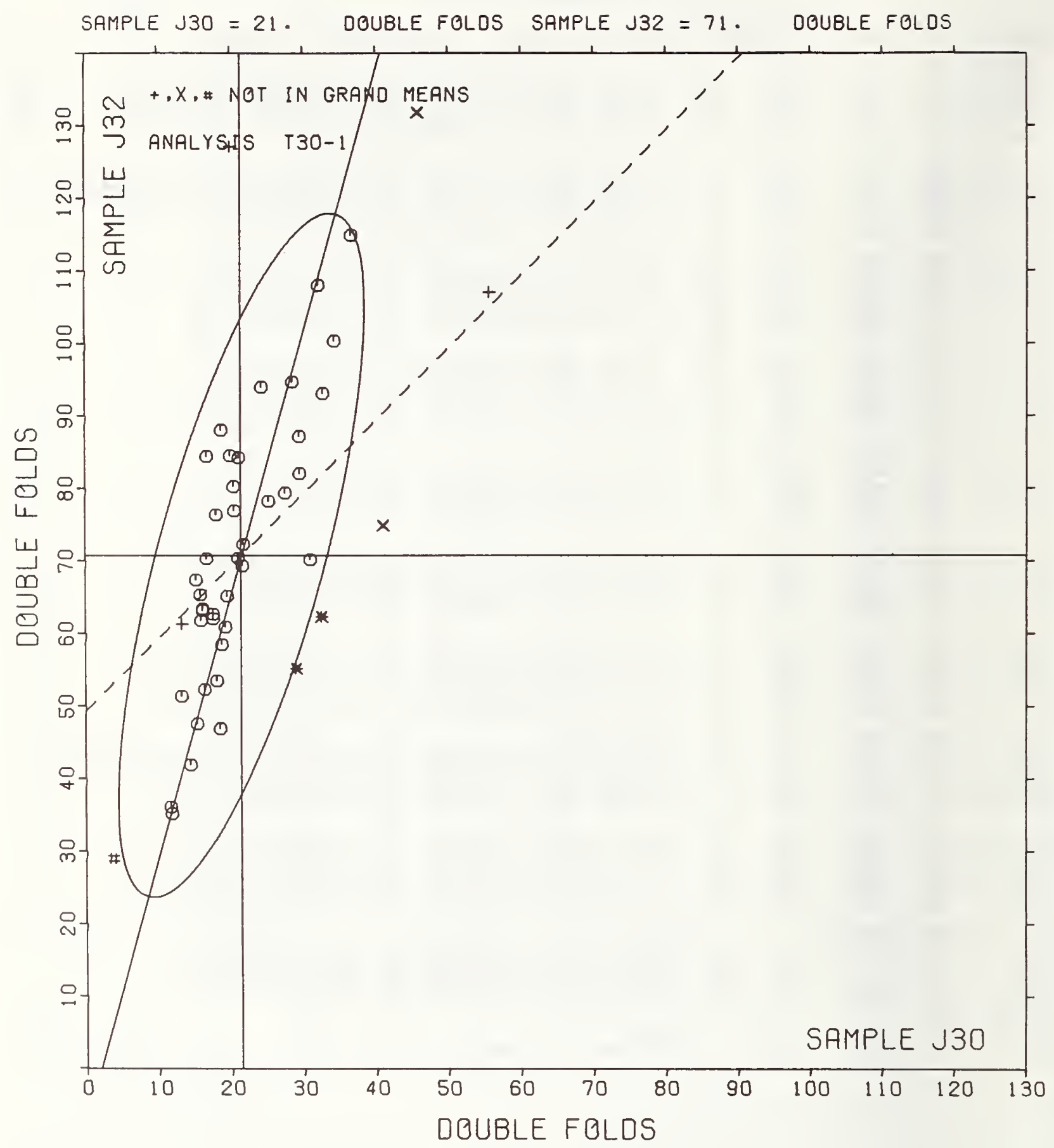




\begin{tabular}{|c|c|c|c|c|c|c|c|c|c|c|c|c|c|}
\hline \multirow{3}{*}{$\begin{array}{l}\text { LAB } \\
\text { CODB }\end{array}$} & \multirow{3}{*}{$\begin{array}{c}\text { SAMPLB } \\
\text { J30 } \\
\text { MBAN }\end{array}$} & \multicolumn{4}{|c|}{ PRINTING } & \multirow{3}{*}{$\begin{array}{c}\text { SAMFLB } \\
\text { J } 32 \\
\text { MEAN }\end{array}$} & \multirow[b]{2}{*}{86 GRAM } & \multicolumn{3}{|c|}{ PRINTING } & \multirow{3}{*}{$\begin{array}{r}\text { TEST } \\
\text { VAR }\end{array}$} & \multirow{2}{*}{ D. } & \multirow{2}{*}{15} \\
\hline & & B5 GSAM & 8 PBR & $\triangle R B$ ME & & & & (9BR & $A R B$ & & & & \\
\hline & & DBV & $\mathrm{N} \cdot \mathrm{DBV}$ & SDR & $\mathrm{R}, \mathbf{8 D R}$ & & DBV & $\mathbf{N} \cdot \mathbf{D B V}$ & SDR & R. SDE & & $\mathbf{F}$ & $L A B$ \\
\hline L2OOM & 1.202 & .0096 & .70 & .060 & .44 & 1.799 & .029 & -.23 & .051 & .46 & $30 \mathrm{M}$ & $\sigma$ & L1 OOH \\
\hline I2 OON & 1.196 &. .103 & -.74 & .051 & .37 & 1,813 & .0015 &. .12 & .046 & .42 & $30 \mathrm{~N}$ & $\theta$ & LIOON \\
\hline L205 & 1.313 & .015 & .11 & .151 & 1.09 & 1.809 & -.019 &. .15 & .190 & 1.71 & $30 x$ & $\sigma$ & 2205 \\
\hline L121 & .459 & -.840 & -6.06 & .092 & .67 & $1 \cdot 422$ & -.407 & $-3 \cdot 24$ & .152 & 1.37 & $30 M$ & 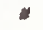 & L121 \\
\hline L1 22 & 1.356 & .057 & .41 & .158 & 1.15 & 1.965 & .137 & 1.09 & .083 & .75 & 304 & $\sigma$ & L122 \\
\hline 1124 & 1.327 & .028 & .20 & .099 & .71 & 1.855 & .026 & .21 & .067 & .60 & $30 \mathrm{~N}$ & $\sigma$ & 1224 \\
\hline$L 150$ & 1.273 & .026 & .019 & .201 & .73 & 1.769 & -.059 & -.47 & .122 & 1.10 & $30 M$ & $\sigma$ & L1 50 \\
\hline L158 & 1.139 & $.15 s$ & -1.15 & .133 & .96 & 1.604 &. .225 & -1.79 & .133 & 1.20 & $30 \mathrm{~N}$ & $\boldsymbol{\theta}$ & 2158 \\
\hline 1162 & 1.279 & -.019 & .014 & - 199 & .72 & 1.795 & .0034 &. .27 & .143 & 1.29 & $30 x$ & $\boldsymbol{\theta}$ & L162 \\
\hline 1263 & $1 \cdot 2 \in 1$ & $.03 e$ & -.27 & .074 & .53 & 1.656 & -.172 & $-1 \cdot 37$ & .116 & 1.05 & $30 \mathrm{~N}$ & $\boldsymbol{\sigma}$ & L1 63 \\
\hline L182M & 1.406 & .107 & .78 & .219 & 1.58 & 1.924 & .095 & .76 & .127 & 2.14 & 304 & $\boldsymbol{\sigma}$ & L182 \\
\hline L185 & 1.304 & .006 & .04 & .139 & 1.01 & 1.919 & .091 & .72 & .077 & .70 & $30 \mathrm{~N}$ & $\boldsymbol{\sigma}$ & 2185 \\
\hline $2190 \mathrm{C}$ & 1.489 & .190 & 1.37 & .132 & .95 & 2.010 & .182 & 1.45 & .155 & 1.39 & $30 \mathrm{~N}$ & $\sigma$ & $2190 \mathrm{C}$ \\
\hline L212 & 1.316 & .017 & .12 & .078 & .57 & 2.830 & .010 & .08 & .097 & .87 & $30 \mathrm{M}$ & $\theta$ & L212 \\
\hline L22 3F & 1.240 & -.058 & -.42 & .119 & .86 & 1.877 & .049 & .39 & .077 & .69 & $30 x$ & $\boldsymbol{\theta}$ & LE23F \\
\hline L230 & 1.217 & .001 & -.58 & .156 & 1.13 & 1.783 & .0045 &. .36 & .097 & .87 & $30 N$ & $\boldsymbol{\sigma}$ & L2 30 \\
\hline L231 & 1.557 & $.25 e$ & 2.86 & .085 & .61 & 2.047 & .219 & 1.74 & .209 & .98 & $30 \mathrm{M}$ & $\theta$ & L231 \\
\hline L232 & 1. 508 & .220 & 1.51 & .080 & .58 & 1.965 & .137 & 1.09 & .058 & .52 & $30 \mathrm{~N}$ & $\sigma$ & L2 32 \\
\hline 1236 & 1.201 & -.057 & -.70 & .067 & .49 & 1.788 & -.041 & -.32 & .107 & .97 & $30 \mathrm{~N}$ & $\sigma$ & .2236 \\
\hline L238A & 1.113 &. .185 & $-1 \cdot 34$ & .069 & .50 & 1.703 &. .125 & -1.00 & .090 & .81 & $30 \mathrm{~N}$ & $\boldsymbol{\theta}$ & L238A \\
\hline L2 $38 B$ & 1.169 & .0125 &. .93 & .214 & .83 & 1.818 & .0011 & .008 & .108 & .97 & $30 \mathrm{D}$ & $\theta$ & L238B \\
\hline 1243 & 1.405 & .107 & .77 & .164 & 1.19 & 1.885 & .056 & .45 & .122 & 1.10 & $30 D$ & $\theta$ & 1243 \\
\hline 1254 & 1.048 &. .250 & $-1 \cdot 81$ & .132 & .95 & 1.510 & -328 & -2.53 & .213 & 1.92 & $30 x$ & $\boldsymbol{\theta}$ & L254 \\
\hline 1262 & 1.192 & .106 & .077 & .090 & .65 & 1.780 & .049 & .39 & .104 & .94 & $30 \mathrm{~N}$ & $\boldsymbol{\theta}$ & L262 \\
\hline 1274 & 1.488 & .290 & 2.37 & .200 & 1.45 & 1.996 & .168 & 1.33 & .072 & .65 & $30 \mathrm{~N}$ & $\theta$ & L274 \\
\hline L275 & 1.212 & .0086 & -.62 & .093 & .68 & 1.917 & .089 & .71 & .095 & .86 & $30 \mathrm{~N}$ & $\theta$ & L275 \\
\hline 1278 & 1.065 &. .233 & -1.68 & .078 & .56 & 1.529 & -.299 & $-2 \cdot 38$ & .126 & 1.14 & $30 \mathrm{C}$ & $\theta$ & 1278 \\
\hline 1279 & 1.226 & .0072 & -.52 & .124 & .89 & 1.771 & .057 & -.46 & .169 & 1.52 & $30 \mathrm{~N}$ & $\boldsymbol{\sigma}$ & 1279 \\
\hline L2 $85 A$ & 1.301 & .003 & .02 & .099 & .72 & 1.878 & .050 & .40 & .090 & .81 & $30 \mathrm{~N}$ & $\sigma$ & L2 85A \\
\hline $1285 B$ & 1.255 & .0044 &. .32 & .203 & 1.47 & 1.908 & .080 & .63 & .138 & 1.25 & $30 \mathrm{~N}$ & $\sigma$ & L285B \\
\hline 1299 & 1.259 & .039 & $\ldots 28$ & .120 & .80 & 1.745 & -.083 & .667 & .247 & 1.33 & $30 \mathrm{~N}$ & $\sigma$ & 1299 \\
\hline 1.320 & 1.180 & .118 & .05 & .213 & 1.54 & 1.705 & .0123 & .98 & .152 & 1.37 & $30 \mathrm{~N}$ & $\theta$ & L320 \\
\hline L326N & 1.150 & .0145 & -2.07 & .221 & 1.60 & 1.695 &. .134 & -1.06 & .152 & 1.37 & $30 \mathrm{~N}$ & $\theta$ & L326N \\
\hline L339 & 1.066 & -.232 & -1.68 & .285 & 2.06 & 1.664 & -.0264 & $-1 \cdot 31$ & .112 & 1.01 & $30 N$ & $\theta$ & L339 \\
\hline 13664 & 1.312 & .015 & .14 & .250 & $1 \cdot 81$ & 1.882 & .054 & .43 & .109 & .98 & $30 \mathrm{~N}$ & $\sigma$ & L3664 \\
\hline 1388 & 1.516 & .218 & 1.57 & .283 & 2.05 & 1.871 & .042 & .34 & .059 & .53 & $30 \mathrm{~N}$ & $\boldsymbol{\theta}$ & L388 \\
\hline L390 & 1.291 & .008 & .006 & .130 & .94 & 1.894 & .066 & .52 & .100 & .90 & $30 \mathrm{~N}$ & 6 & L390 \\
\hline$L 393$ & 1.473 & $.17 E$ & 1.26 & .191 & $1 \cdot 38$ & 1.786 & .0043 & -.34 & .093 & .84 & $30 \mathrm{H}$ & $\sigma$ & 2393 \\
\hline L3964 & 8.364 & .065 & .47 & .304 & 2.20 & 1.737 & .0091 & $\ldots 73$ & .067 & .60 & $30 N$ & $\theta$ & L396M \\
\hline L565 & 1.420 & .122 & .88 & .169 & 1.22 & 1.960 & .131 & 1.05 & .131 & 1.18 & $30 N$ & $\theta$ & L565 \\
\hline 1567 & 1.452 & .153 & 1.21 & .131 & .94 & 1.895 & .067 & .53 & .137 & 1.23 & $30 N$ & $\sigma$ & 2567 \\
\hline 1589 & 1.215 & .004 &. .60 & .082 & .60 & 2.842 & .022 & .10 & .078 & .70 & $30 \mathrm{~N}$ & $\boldsymbol{\sigma}$ & 1589 \\
\hline L599 & 1.482 & .183 & $1 \cdot 32$ & .087 & .63 & 1.834 & .005 & .04 & .109 & .98 & $30 \mathrm{C}$ & $\theta$ & L599 \\
\hline 1622 & 1.616 & .317 & 2.29 & .200 & 1.45 & 2.090 & .262 & 2.09 & .181 & 1.63 & $30 \mathrm{M}$ & $\theta$ & L622 \\
\hline 2670 & 1.265 & .025 & -.21 & .056 & .40 & 1.939 & .111 & .88 & .074 & .67 & $30 \mathrm{~N}$ & $\sigma$ & L670 \\
\hline
\end{tabular}

GR. JEAH - 1.298 LOO(20) ROLD SD MBANS. 139 LOG(10) POLD
ORAND MEAN - 1.828 LEG(10) FELD

SD AP MBANS - .126 I GO(10) FELD
AVBRAGB SDR -

TBST DETBRHINATIENS - 15 44 LABS IN GRAND MBANS

$$
\begin{aligned}
& 44 \text { LABS IN GD } \\
& .111 \text { LGG(10) RELD }
\end{aligned}
$$

\begin{tabular}{|c|c|c|c|c|c|c|c|c|c|c|c|c|}
\hline L182S & 1.265 & .034 &. .24 & .119 & .86 & 2.251 & .323 & 2.57 & .081 & .73 & $30 \mathrm{~s}$ & - L282S \\
\hline L190D & 1.251 & -.048 & -.34 & .136 & .98 & 2.175 & .347 & 2.77 & .132 & 1.19 & $30 \mathrm{~s}$ & - L190D \\
\hline L2 80 & 1.104 &. .194 & -1.40 & .127 & .92 & 1.779 & .049 & -39 & .086 & .78 & $30 \mathrm{I}$ & - L280 \\
\hline $1326 \mathrm{~s}$ & 1.183 & -.116 & -.84 & .255 & 1.85 & 2.057 & .228 & 1.82 & .192 & 1.73 & $30 \mathrm{~s}$ & - L3268 \\
\hline I396S & 1.611 & .312 & 2.25 & .384 & 2.78 & 1.955 & .127 & 2.01 & .251 & 2.27 & $30 T$ & - L3968 \\
\hline
\end{tabular}

The ISU (Internationel Stenderds Oraenlzetion) 1s propoilng that MIT rolding endurance be reported as the loceritnm (to the hase 10 ) of the double fold Instead of the double rold a in the past.

Analysis T30-1 in thio report la the same as in the past -1 th no changes. The analysis, T30-2, shows the date a the ISG proposes. Thlo anelyele uses the rer dete reported ror T30-1. The rel date ere converted to the logerithm (base in) as shown in tbe exeaple to the right, and then the meen of the converted date is calculated and reported a Ise rolding endurance.
Ray data

(Polding number

in double rolde)

$\begin{array}{ll}207 & 2.32 \\ 166 & 2.22 \\ 151 & 2.18 \\ 332 & 2.52 \\ 260 & 2.41 \\ 137 & 2.14 \\ 199 & 2.30 \\ 230 & 2.36 \\ -0- & -0.0 \\ 210 & 2.31\end{array}$


DATA IS LOO(BASB 10 ) OP TBB DOUBLB FELD NBASORBMENT

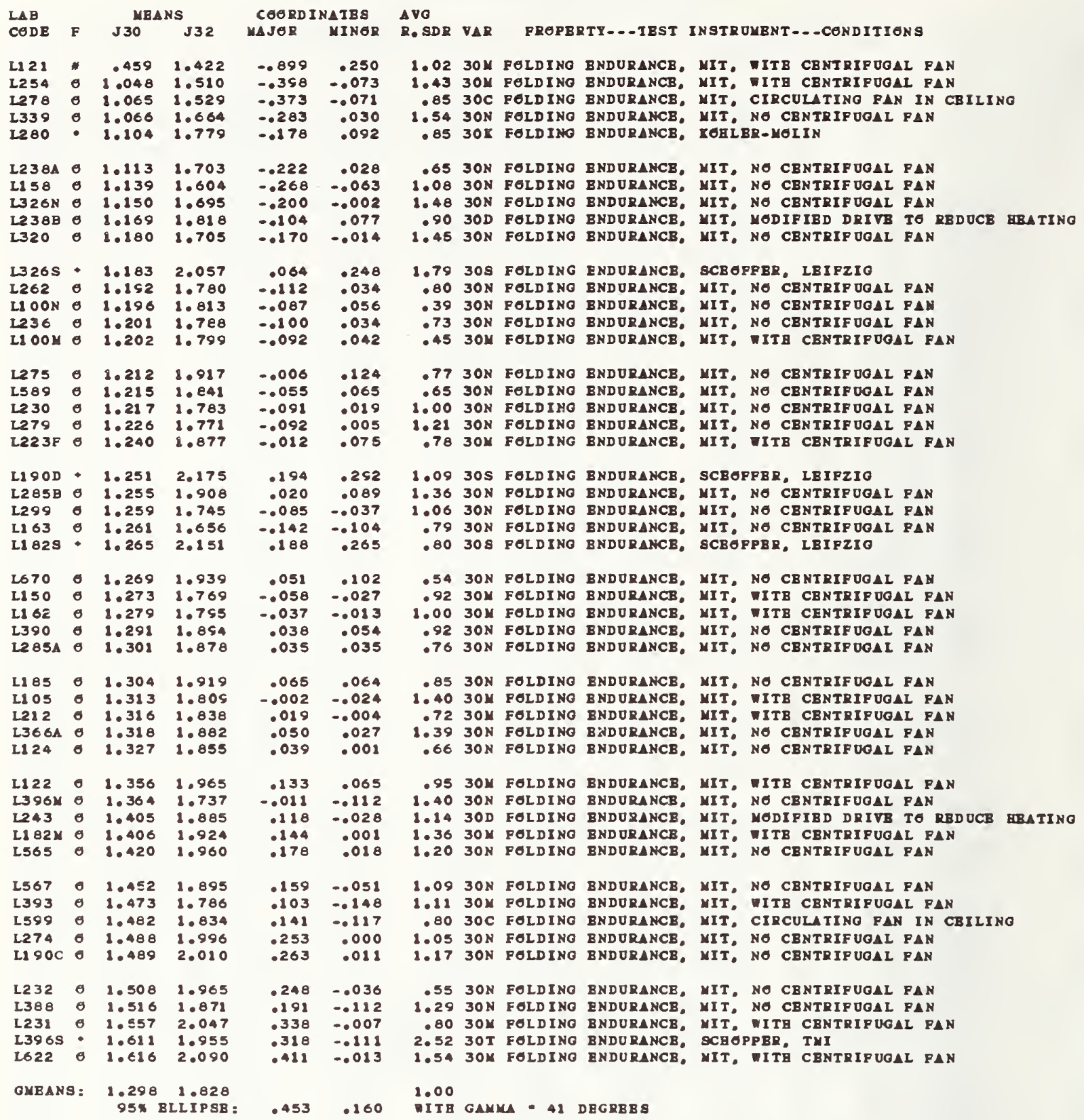


FOLIING ENDURANCE (MIT)

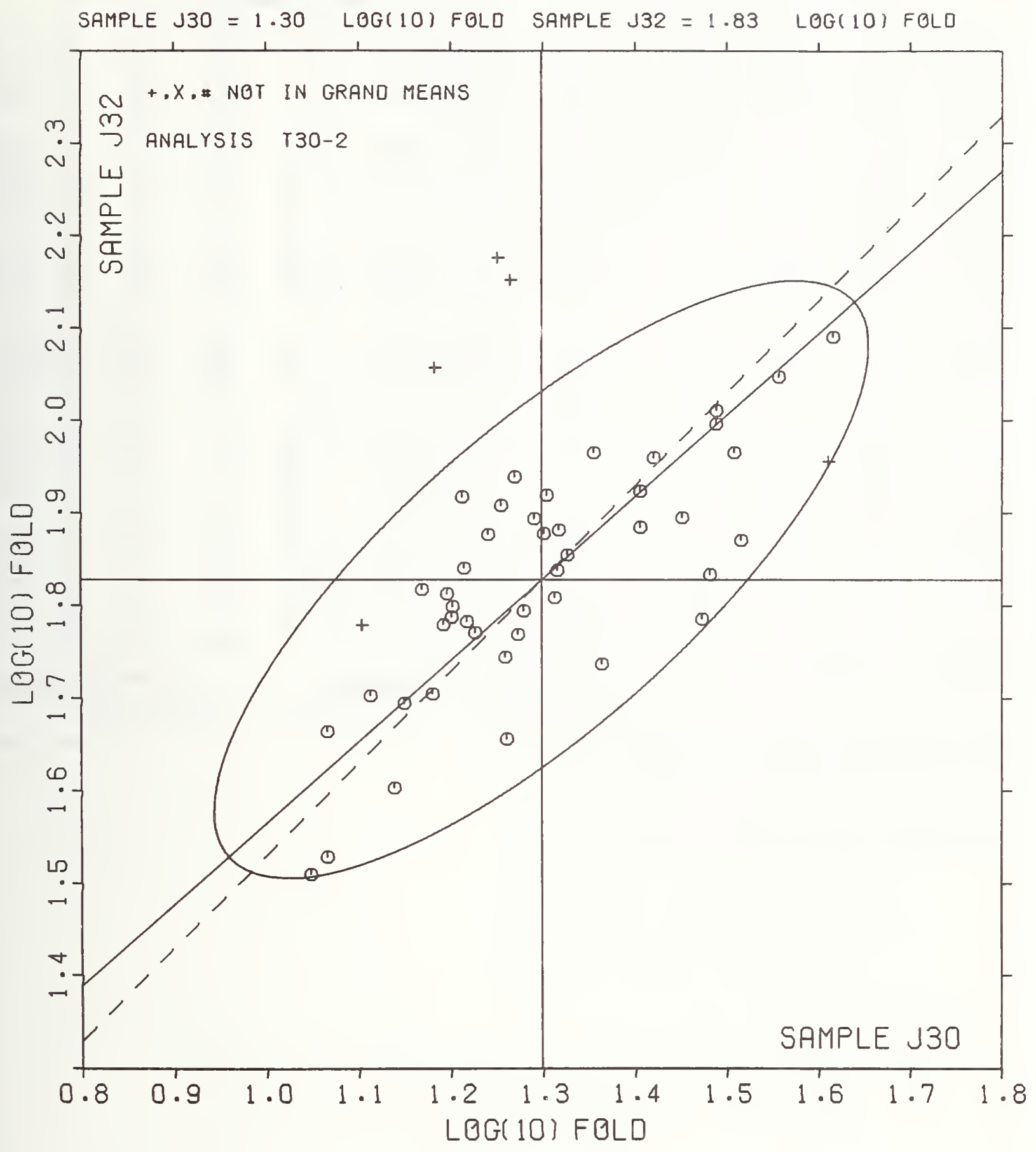


RESULTS BXPRBSSBD IN SIANDAED GORLBY ONITA: MILLIGRAMS FER 1 IX INCH SPBCIMBN (ACTUAL LBNOTB 3.5 INCHBS)

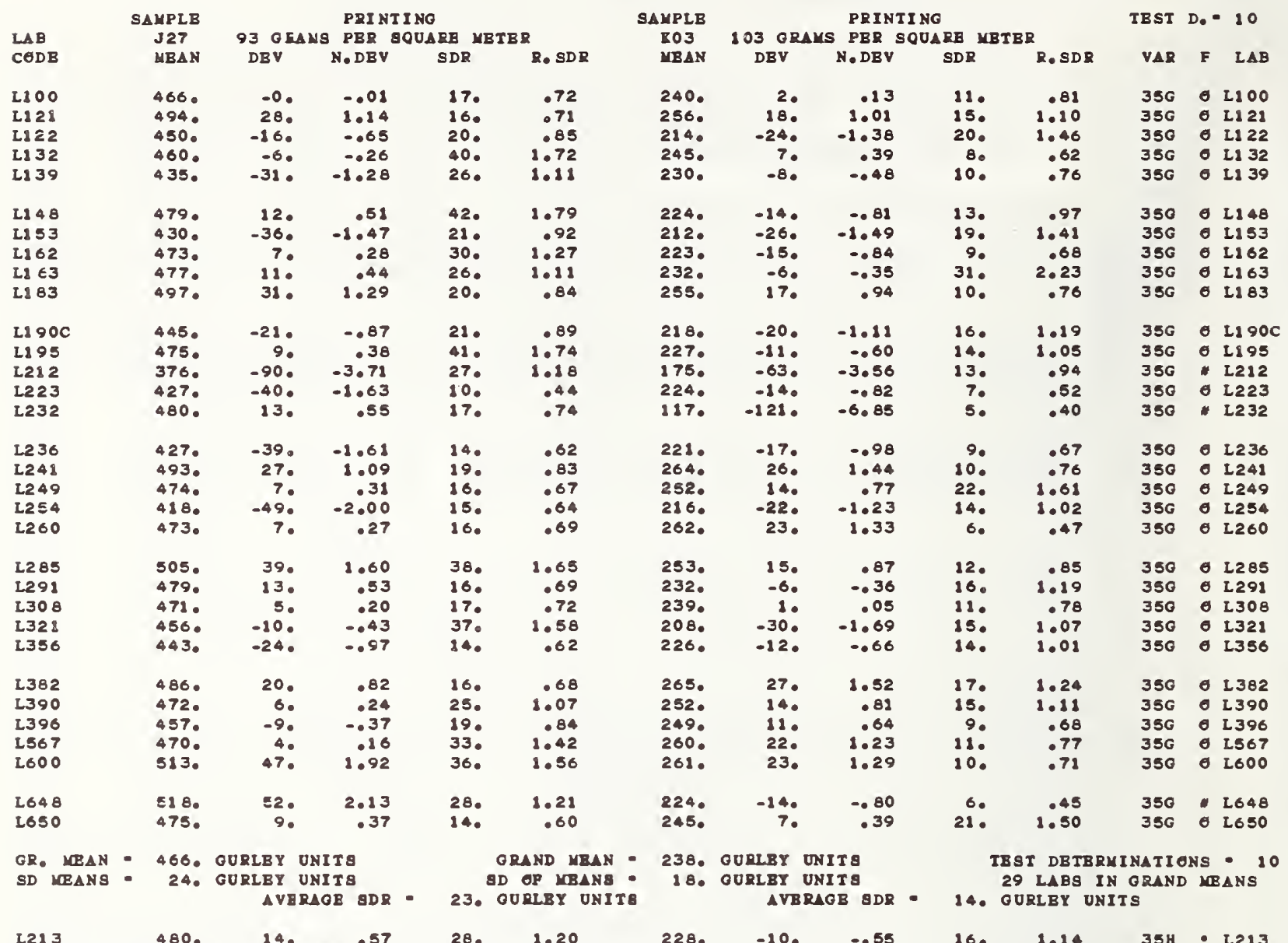

TOTAL NUMBER OF LABORATEEIBS EBPORTING: $33^{1.2}$

Best values: $\mathrm{J} 27470+40$ Gurley units

K03 $240 \mp 30$ Gurley units

The following laboratories were omitted from the

grand means because of extreme test results: 212 ,

232,648 . 


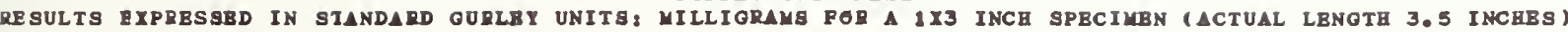

LAB MBANB

$\begin{array}{lllll} & & & \end{array}$

1212 376. 175

$\mathrm{L} 2540418.216$.

L223 427,224 .

L236 6427.221.

L153 430.212.

1390435.230.

L356 o 443. 226.

L19OC 445. 218.

L122 450.214.

L321 0 456. 208.

1396 - 457.249

13320460 . 245.

L100 o 466. 240 .

L567 470 . 260 .

1308 471.

$1390 \circ 472.252$.

$1260 \quad 473.262$.

L162 0473.223.

12490474.252.

1650 o 475.245.

L195 0475.227

$1630477^{\circ} 232$.

L48 0 479. 224.

12019

$123248 C .117$.

$\mathrm{L213} 448 \mathrm{O} .228$

L382 686.265.

L241 8 493. 264.

L121 o 494. 256.

L183 8497.255.

L285 6 505. 253.

16000 513. 261.

L648. 518.224.

GIBANS :

466.238.
COORDINATES AVO

MAJOR MINOR R. SDP VAR PREFBRTY-_-IBST INGTRUMBNT-_-CENDITIGNS

$-110$.

$-63$.

$-41$.

$-42$.

$-4.4$

$-31$.

$-26$.

$-28$.

$-27$.

-25 .

$-2$.

-2 .

1.

15.

13.

18.

-2 .

14.

11.

2. -14.

3. -19.

7. -12 .

$-54 .-109$.

6. -16.

31.12.

36. 7.

33.

35.

41.

52.

36,

$-40$.

$95 \%$ BLLIP 8B:
-0.
-3 .

$-8$.

$-6$.

1.06350 ITIPENBS , OURLEY (UNITS: MG/1X3-ACTUALLY 3.5- TBST PIBCB) . 83350 STIPPNBSS, GURLBY (UNITS: MG/1X3-ACTUALLY 3.5- TBST PIBCB)

- 48350 STIPPNBSS. GURLBT (UNIT 8; MG/1X3-ACTCALLY 3.5- TBST PIBCB)

.64350 STIPPNBSS, OURLBY (UNIT S: MO/1X3-ACTUALLY 3.5- TEST PIBCB)

1.17350 STIPFNBS, OULBY (UNITS: MO/1X3-ACTUALLY 3.5-TBST PIBCB)

.94350 STIFFNESS, OURLBY (UNITS: MO/1X3-ACTUALLY 3.5- TBST PIBCB)

81350 8TIFFNB85, OURLBY ( ONITS: MO/1X3-ACTUALLY 3.5- TBST PIBCB)

1.04360 STIPPNBS, OURLEY (UNIT : NO/1 X3-ACTUALLY 3.5- TEST PIBCE)

1.16350 STIPPNBSS, GURLBY (UNITS: NO/1X3 - ACTUALLY 3.5-TBST PIBCB)

1.33 350 STIPFNB8S, OURLBY (UNITS: MO/1X3-ACTUALLY 3.5-TBST PIBCB)

.76350 STIPPNB89, GURLBY (UNITS: MO/1X3-ACTUALLY 3.5-TBST PIBCE)

1.17350 STIPFNBSS, OURLBY (UNITS: MO $/ 1 \times 3$-ACTUALIY 3.5-TBST PIBCE)

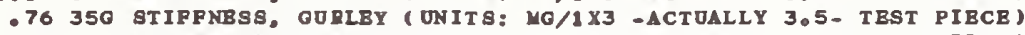

1.09350 STIFFIBA9, OURLIY (UNITS: LG/1X3-ACTUALLY 3.5- TBST PIECE)

-75 350 STIPFNBS, GDRLBY ( UNITS: MO/1X3-ACTUALLY 3.5- TBST PIBCB)

1.09 $35 G$ STIFFNBS, OURIET (UNITS: HO/1Z3-ACTOALLY 3.5- TEST PIBCB)

.58 350 STIPFNBSS, GURLBY (UNITS: MO/1Z3- $\triangle$ CTOALLY 3.5- TBST PIBCE)

.98350 STIFFNBS9, OURLBY (UNITS: MO/1X3-ACTUALIY 3.5-TBST PIBCB)

1.14 350 8TIFFIBSS, OURLBY ( ONITS: MG/1 X3 - ACT UALLY 3.5- TBST PIECE)

1.05350 STIFFNBS, OURLEY (UNITS: MO/1X3-ACTUALIY 3.5-TEST PIBCB)

1.40 350 STIRFNBSS, GURLE (UNITS: MG/1XJ-ACTUALIY 3.5- TEST PIBCE) 1.67350 STIPFXBSs, GURLBY (UNITS: MG/1X3-ACTUALLY 3.5- TBST PIBCB)

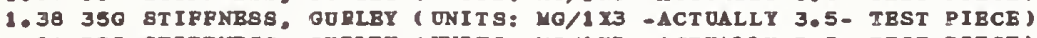

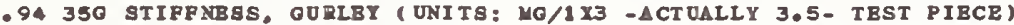

.57350 SIIFPNBS, OURLBY (UNITS: MO/1 X3 - $\triangle$ CTOALIY 3.5- TEST PIBCE)

1. 1735.7 STIRPNBSS, OURLBY ( UNITS: MO/1X3 TBST PIBCB), 20 C, 65\% RH .96 360 STIFPNBSS, GURLBY (UNITS: MO/1X3-ACTUALLY 3.5- TBST PIECB) -8n 350 STIFPNBSS, GURLBY (UNIT: MO/1X3-ACTUALLY 3.5- TBST PIBCB) .90350 STIPFNBS, GURLBY (UNIT S: MO/1X3-ACTUALLY 3.5- TBST PIBCB) .80350 ST T.PFNBSS, GJRLBY ( ONITS: MO/1X3-ACTUALLY 3.5- TBST PIBCB)

1.25 350 STIPFNB8, GURLBY ( UNITS: MG/1 X3-ACTOALLY 3.5-TEST PIBCE) 1.14 350 STIPFHBS8, GURLBY (UNITS: MO/1X3-ACTULLY 3.5-TEST PIBCE) $.8335 G$ STIPFNES8, GORLBY ( ONITS: MG/1 X3-ACTOALLY 3.5-TEST PIBCE)

1.00

28. VITH GAMM - 32 DBGRBES 
STIFFNESS, GURLEY

SAMPLE J27 $=466$. GURLEY UNITS SAMPLE KO3 $=238$. GURLEY UNITS

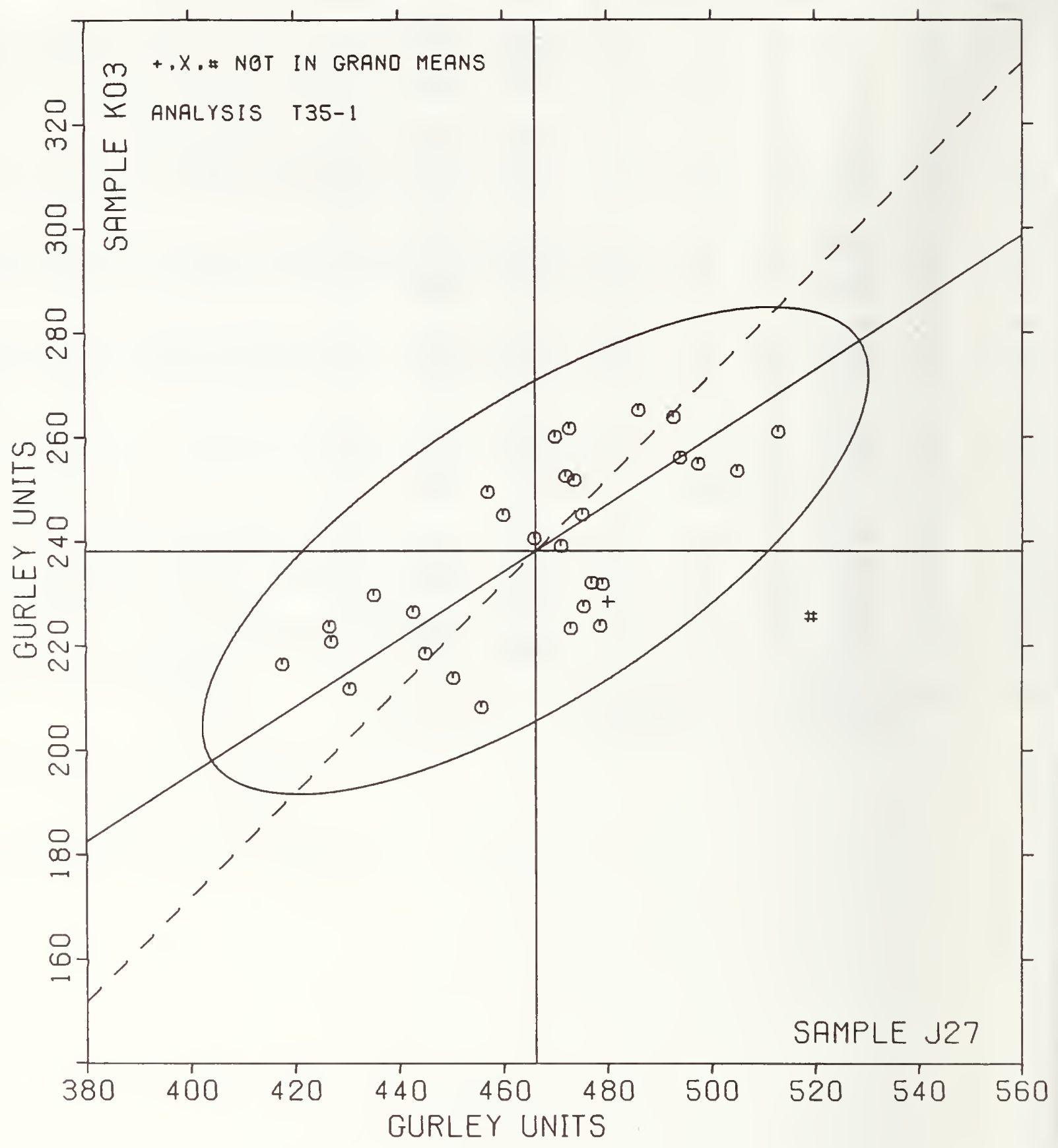




$$
\text { TARBR CTIPENIIE }
$$

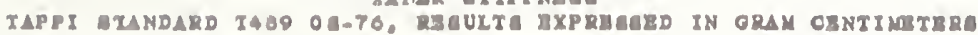

\begin{tabular}{|c|c|c|c|c|c|c|c|c|c|c|c|c|c|}
\hline \multirow{3}{*}{$\begin{array}{l}\mathrm{LAB} \\
\operatorname{CODB}\end{array}$} & $\triangle \triangle M P L$ & \multicolumn{4}{|c|}{ SRAPT INVELOPB } & \multicolumn{5}{|c|}{ CONVBRTBR KRAPT } & \multirow[t]{2}{*}{$\operatorname{TB} B x$} & \multirow[t]{2}{*}{ D. } & \multirow[t]{2}{*}{10} \\
\hline & 863 & 124 OEA & $18 \mathrm{PBR}$ s & $\triangle R B$ & & 168 & $1680 \mathrm{OL}$ & $\angle 8 P B R$ & $\triangle A B B$ & & & & \\
\hline & MBAN & DBV & N. DBV & SDR & R. BDR & MBAN & $\mathbf{D g V}$ & N.DBV & $\mathbf{D D R}$ & R. SDR & VAR & $F$ & LAB \\
\hline L107A & 18.90 & .42 & .64 & .81 & 1.06 & 26.47 & .0 .04 & .0 .04 & 1.03 & .93 & $36 \mathrm{~T}$ & $\theta$ & L107A \\
\hline 1222 & 17.70 & .078 & -1.20 & .60 & .78 & $27 \cdot 13$ & .61 & .60 & 1.32 & 1.19 & $36 I$ & $\theta$ & $\mathbb{1 1 2 2}$ \\
\hline L123 & $18 \cdot 20$ & -.28 & -.43 & 1.34 & 1.49 & 25.30 & $-1 \cdot 21$ & -2.18 & 2.42 & 1.29 & $36 \mathrm{~T}$ & $\theta$ & L123 \\
\hline 1226 & 18.67 & .19 & .29 & .55 & .73 & 25.12 & $-1 \cdot 38$ & $-1 \cdot 35$ & $\$ .07$ & .97 & $36 \mathrm{~T}$ & $\boldsymbol{\theta}$ & L126 \\
\hline L1 50 & 19.30 & .82 & $1 \cdot 25$ & 8.06 & 1.39 & 28.20 & 1.69 & 1.66 & 1.55 & 1.41 & $36 \mathrm{~T}$ & $\theta$ & L150 \\
\hline L158 & 1.84 & -16.64 & -25.47 & .17 & .23 & 2.97 & $-23 \cdot 54$ & $-23_{6} 07$ & .89 & .18 & $36 \mathrm{~T}$ & 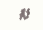 & L258 \\
\hline L163 & 39.05 & .57 & .87 & .64 & .84 & 27.45 & .94 & .93 & 1.21 & 1.10 & $36 \mathrm{~T}$ & $\boldsymbol{\theta}$ & 1163 \\
\hline $\operatorname{L173B}$ & 17.46 & -1.02 & -1.57 & .54 & .71 & 25.56 & .15 & .15 & . 32 & .29 & $36 \mathrm{~T}$ & $\theta$ & L273B \\
\hline L1 82 & 18.57 & .09 & .13 & .71 & .93 & 26.36 & -.85 & -.24 & 1.18 & 1.07 & $36 \mathrm{~T}$ & $\theta$ & L 182 \\
\hline 1207 & 18.03 & -.45 & -.69 & $1 \cdot 21$ & 1.59 & 26.40 & -.10 & -.10 & 1.65 & 1.50 & $36 \mathrm{~T}$ & $\theta$ & L207 \\
\hline L21 2 & 18.03 & -.45 & .069 & .59 & .78 & 26.78 & .27 & .27 & .87 & .79 & $36 \mathrm{~T}$ & $\sigma$ & L222 \\
\hline L228 & 18.50 & .02 & .03 & 1.08 & 1.42 & 24.90 & -1.62 & -1.57 & 1.10 & 1.00 & $36 T$ & $\theta$ & L229 \\
\hline 1230 & 20.20 & 1.72 & 2.63 & 1.23 & 8.61 & $31 \cdot 20$ & 4.69 & 4.60 & 3.01 & 2.74 & $36 \mathrm{~T}$ & $\omega$ & L230 \\
\hline L236 & 17.80 & -.68 & -1.05 & .55 & .72 & 25.18 & $-8 \cdot 33$ & -1.30 & 1.32 & 1.20 & $36 \mathrm{~T}$ & $\theta$ & L236 \\
\hline 1242 & 20.02 & 1.54 & $2 \cdot 35$ & .44 & .57 & 30.14 & 3.63 & 3.56 & .74 & .63 & $36 \mathrm{~T}$ & $*$ & $\mathrm{L242}$ \\
\hline L243 & 18.45 & .003 & -.05 & .76 & 2.00 & $27 \cdot 30$ & .79 & .78 & .82 & .75 & $36 T$ & $\theta$ & L243 \\
\hline L2 60 & 18.93 & .45 & .68 & .40 & .53 & 28.17 & 1.66 & 1.63 & .66 & .60 & $36 \mathrm{~T}$ & $\theta$ & L260 \\
\hline$L 262$ & 18.75 & .27 & .41 & .63 & .83 & 27.90 & 1.39 & 1.37 & .97 & .88 & $36 \mathrm{~T}$ & $\theta$ & L262 \\
\hline 2274 & 19.05 & .57 & .87 & .69 & .90 & 26.30 & -.22 & -20 & .63 & .57 & $36 \mathrm{I}$ & $\theta$ & L274 \\
\hline 2281 & 18.19 & -.25 &. .44 & .55 & .72 & 27.48 & .91 & .89 & 1.26 & 1.14 & $36 \mathrm{~T}$ & $\theta$ & โ281 \\
\hline L290 & 18.90 & .42 & .64 & .52 & .68 & 26.45 & .06 & .05 & 1.44 & $1 \cdot 31$ & $36 \mathrm{~T}$ & $\theta$ & L290 \\
\hline L31 3 & 17.80 & .068 & -1.05 & .42 & .55 & 26.40 & -.11 & .10 & .97 & .88 & $36 \mathrm{~T}$ & อ & L.313 \\
\hline L318 & 18.60 & .12 & .28 & .94 & 1.24 & 25.42 & .08 & .08 & 1.01 & .92 & $36 T$ & $\theta$ & 2328 \\
\hline 2321 & 17.11 & $-1 \cdot 37$ & -2.10 & .63 & .86 & 24.36 & -2.15 & $-2,10$ & .90 & .81 & $36 \mathrm{~T}$ & $\theta$ & L321 \\
\hline L.324 & 19.50 & 1.02 & 1.56 & .45 & .59 & 27.56 & 1.05 & 1.03 & 1.09 & .99 & $36 \mathrm{~T}$ & $\theta$ & 2324 \\
\hline L339 & 54.40 & 35.92 & 54.97 & 2.87 & 3.76 & 77.10 & 50.59 & 49.59 & 3.17 & 2,88 & $36 \mathrm{~T}$ & $*$ & L339 \\
\hline 1388 & 26.76 & 8.28 & 12.67 & 2.79 & 3.66 & 31.35 & 4.84 & 4.75 & 1.96 & 1.78 & $36 \mathrm{~T}$ & $*$ & L388 \\
\hline L442 & 17.55 & -.93 & -1.43 & .60 & .79 & 25.55 & -.96 & -0.94 & .89 & .81 & $36 \mathrm{~T}$ & $\boldsymbol{\theta}$ & L442 \\
\hline L484 & 18.08 & -.40 & -.62 & .45 & .59 & 25.29 & $-1 \cdot 22$ & $-1 \cdot 19$ & .62 & .57 & $36 \mathrm{~T}$ & $\theta$ & L484 \\
\hline 2570 & $18 \cdot 30$ & -.18 &. .28 & .95 & 3.24 & 26.50 & -.01 & .01 & 1.72 & 1.56 & $36 \mathrm{~T}$ & $\theta$ & L570 \\
\hline LS 80 & 19.20 & .72 & 1.10 & .63 & .83 & 25.90 & -.61 & -.59 & .88 & .80 & $36 \mathrm{~T}$ & $\theta$ & L580 \\
\hline L616 & 19.40 & .92 & 1.40 & .97 & 1.27 & 27.30 & .79 & .78 & .67 & .61 & $36 \mathrm{~T}$ & $\theta$ & 1616 \\
\hline 2651 & 19.50 & 1.02 & 1.56 & 2.27 & 2.98 & 27,40 & .89 & .88 & 2.27 & 2.06 & $36 \mathrm{~T}$ & $\theta$ & L651 \\
\hline
\end{tabular}

GR. MEAN - 18.48 TABBR ONITS

SD MRANS = 65 TABBP TNITS

$$
\triangle \text { VIRAGB SDR = }
$$

GRIND IEAN - 26.52 TAEBR UNITS SD $6 R$ MRANS = 3.02 TABER TNITS AVBRAGB SDR $=1.10$ TABBR ONITS

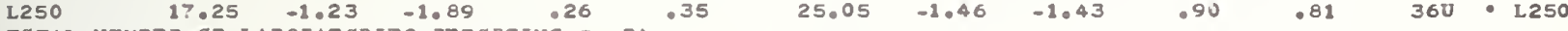

TOTAL NOMSER OF LABOEATORIBS FBPERIING * 34

Best values: $\mathrm{B} 6318.5+0.9$ Taber units

A65 $26.5 \pm 1.6$ Taber units
}

The following laboratorles were omitted from the

grand means because of extreme test results: 230 ,

$242,388$.

Data from the following laboratorles appear to be

off by a multiplicative factor: $158,339$. 
TAPPI SIANDARD TA89 OS-76, RBSULTS BXPRESSED IN GRAM CBNTIMETBRS

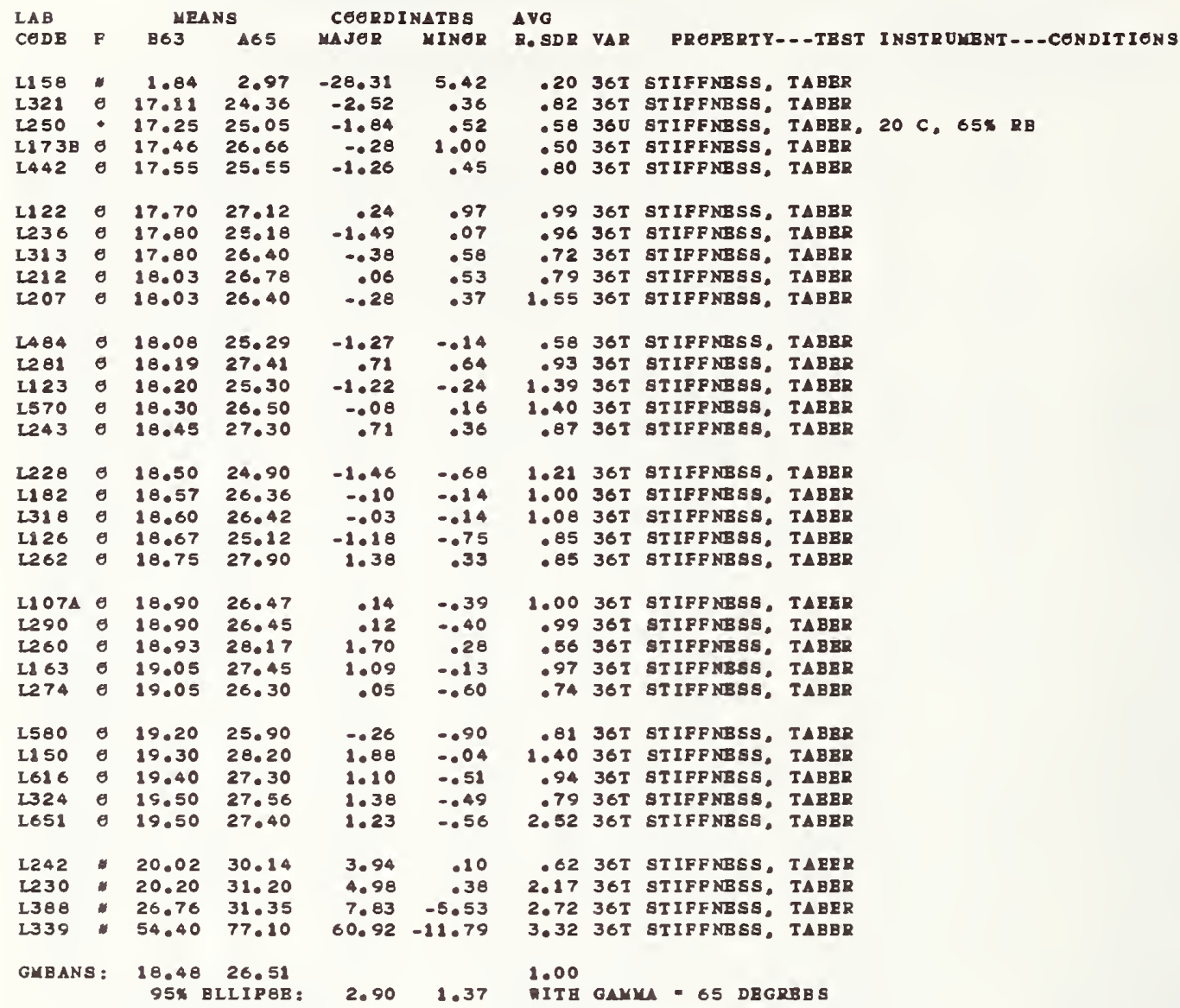


STIFFNESS, TABER

SAMPLE B63 $=18.5$ TABER UNITS SAMPLE A65 $=26.5$ TABER UNITS

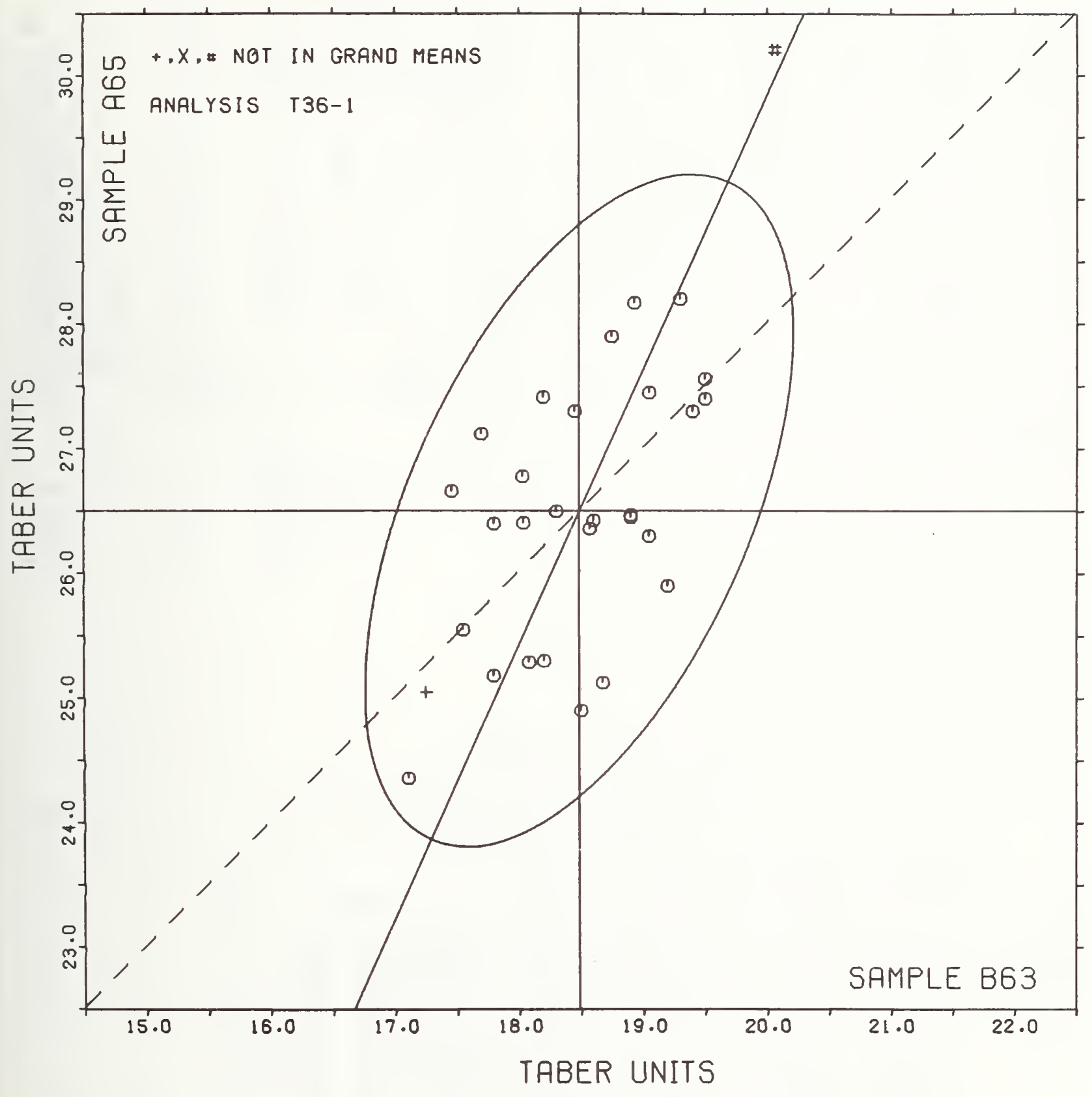




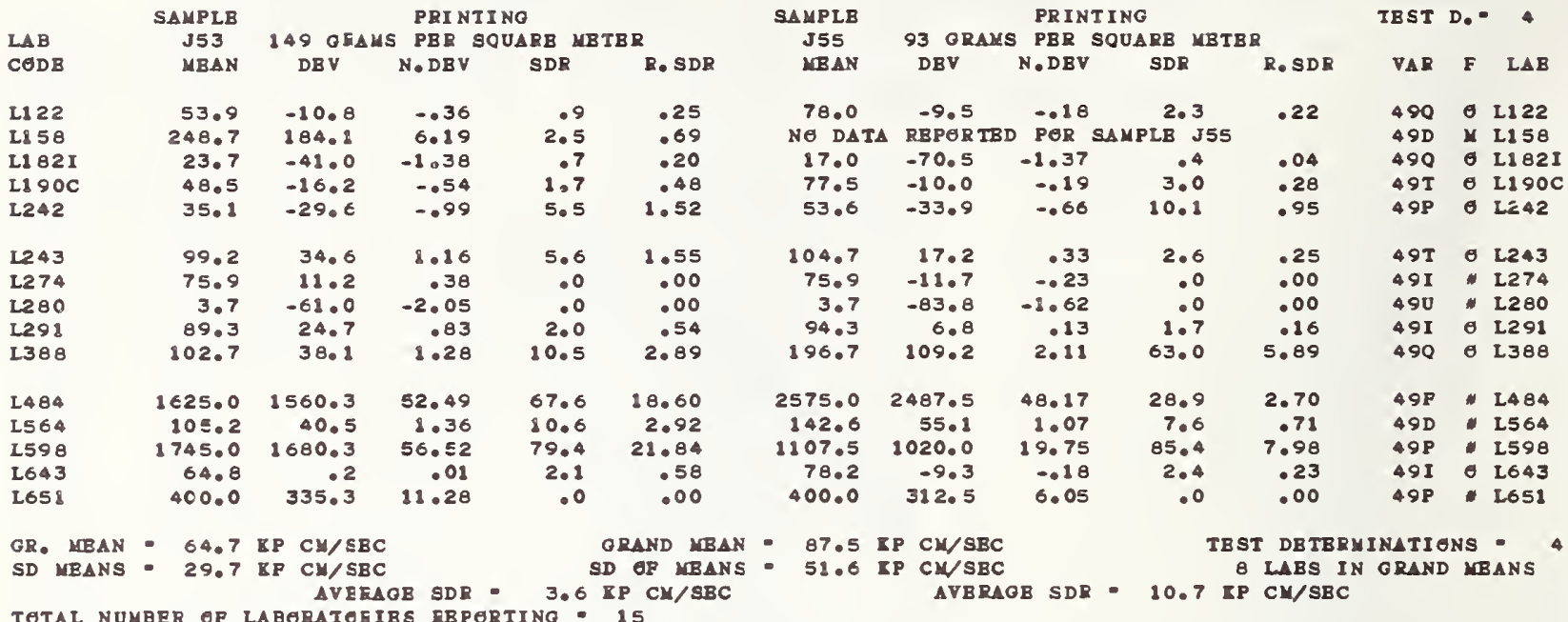

Data from the following laboratories were omitted

from the grand means because no viscosity values

were reported: $274,484,564,598,651$.

Data from the following laboratories were onitted

from the grand theans because the values were

outside the range of the instrument: 280 .

Lab Meane in ca/sec

\begin{tabular}{lll} 
& J53 & \multicolumn{1}{c}{ J55 } \\
L274 & 75.88 & 75.88 \\
L484 & 162.5 & 257.5 \\
L564 & 105.2 & 142.6 \\
L598 & 174.5 & 110.75 \\
L651 & 203.2 & 203.2
\end{tabular}

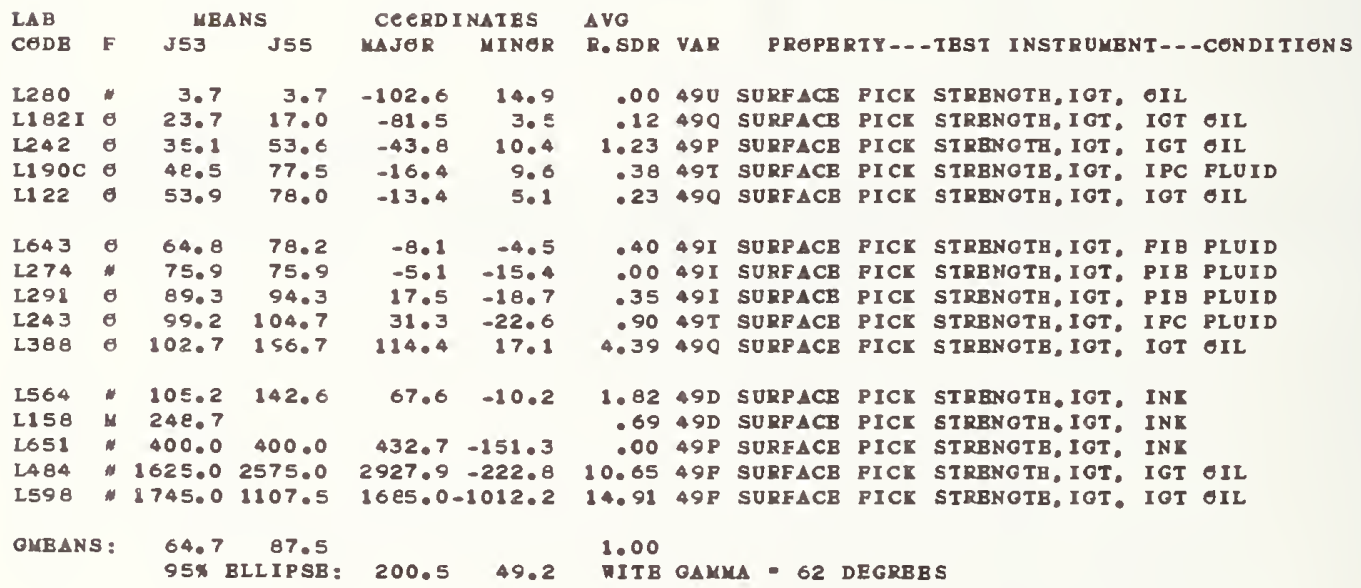


BURPACB PICE STRBNGTB, WAX NOMBBD

IAPPI STANDARD T459 69-75, 8ORPACE STRBNOTB OP PAPBR (

\begin{tabular}{|c|c|c|c|c|c|c|c|c|c|c|c|c|c|}
\hline \multirow{3}{*}{$\begin{array}{l}\text { LAB } \\
\text { CODB }\end{array}$} & \multirow{3}{*}{$\begin{array}{c}\text { BAMPLB } \\
\text { JS3 } \\
\text { MBAN }\end{array}$} & \multicolumn{4}{|c|}{ PRINT ING } & \multirow{3}{*}{$\begin{array}{c}\text { SAMPLB } \\
\text { JS5 } \\
\text { MBAN }\end{array}$} & \multirow[b]{2}{*}{93 ORAME } & \multicolumn{2}{|c|}{ PRINT INO } & \multirow{2}{*}{\multicolumn{2}{|c|}{ IBST }} & \multirow{2}{*}{$D_{0} \cdot$} & \multirow{2}{*}{5} \\
\hline & & 149 ODAMS & 3 PER \& & SQOARB MI & & & & 3 PBR S & $\triangle R B \quad M E$ & & & & \\
\hline & & DBV & N.DBV & SDR & R. SD R & & DBV & N.DBV & SDR & $R_{\bullet}$ SDR & VAR & $\mathbf{F}$ & LAB \\
\hline L105 & 11.00 & .81 & 1.05 & .71 & $1 \cdot 35$ & 21.40 & $-1 \cdot 48$ & -1.08 & .55 & .88 & $50 \pi$ & $\boldsymbol{\theta}$ & L1 OS \\
\hline 1122 & 9.60 & -.55 & .076 & .55 & 1.04 & 13.40 & .52 & .38 & .53 & .88 & $50 \mathrm{~W}$ & 0 & L:22 \\
\hline L158 & 9.60 & -.58 &. .76 & .89 & 1.70 & $12 \cdot 60$ &. .28 & -.21 & .55 & .88 & $50 \%$ & $\theta$ & 2158 \\
\hline L162 & 10.00 & -.19 &. .25 & 1.00 & 1.90 & 13.40 & .52 & .38 & .55 & .88 & $50 \mathrm{~W}$ & $\boldsymbol{\theta}$ & L1 62 \\
\hline LI 734 & 10.60 & .41 & .53 & .55 & 1.04 & 12,20 &. .68 & -.50 & .45 & .72 & $50 \mathrm{~W}$ & $\boldsymbol{\sigma}$ & 21734 \\
\hline L1 82 W & 11.00 & .81 & 1.05 & .00 & .00 & 12.80 & -.08 & .006 & .45 & .72 & $50 \mathrm{w}$ & $\boldsymbol{\theta}$ & 21820 \\
\hline 2183 & 10.00 & -.18 & -.25 & .00 & .00 & 13.20 & .32 & .23 & .34 & 1.35 & $50 w$ & $\boldsymbol{\theta}$ & 2183 \\
\hline 2195 & 10.80 & .61 & .79 & .45 & .85 & 12.80 & .008 & .006 & .45 & .72 & $50=$ & $\boldsymbol{\theta}$ & 2195 \\
\hline 2213 & 10.20 & .01 & .01 & .84 & 1.59 & 13.80 & .92 & .67 & 1.30 & 2.10 & $50=$ & $\boldsymbol{\theta}$ & 1213 \\
\hline 1225 & 5.80 &. .35 & -.31 & .45 & .85 & 13.00 & .12 & .09 & .71 & 1.14 & $50 \mathrm{~W}$ & $\boldsymbol{\theta}$ & L225 \\
\hline$\lcm{228}$ & 9.60 & -.58 & -.76 & .55 & 1.04 & 10.40 & -2.48 & -1.82 & .55 & .88 & 50W & $\theta$ & 1228 \\
\hline L2 30 & 9.20 & .058 & -1.28 & .45 & .85 & 12.40 & .048 & -.35 & .55 & .88 & $50 \mathrm{~W}$ & $\theta$ & 1230 \\
\hline 1236 & 9.80 & -.39 & -.51 & .45 & .85 & 13.40 & .52 & .38 & .55 & .86 & $50 \%$ & $\boldsymbol{\sigma}$ & L236 \\
\hline L243 & 8.40 & -1.79 & .2 .32 & .55 & 1.04 & 9.50 & -3.28 & -2.40 & 1.14 & 1.84 & $50 \%$ & $\theta$ & 2243 \\
\hline 2274 & 10.00 & .029 & -.25 & .00 & .00 & 13.20 & .32 & .23 & .45 & .72 & $50 \%$ & $\boldsymbol{\sigma}$ & 2274 \\
\hline L285 & 11.20 & 1.01 & 1.31 & 1.10 & 2.09 & 14.40 & 1.52 & 1.11 & 1.14 & 1.84 & 50w & $\boldsymbol{\sigma}$ & L285 \\
\hline 1339 & 9.80 & .039 & -.51 & .45 & .85 & $13 \cdot 20$ & .32 & .23 & 1.10 & 1.77 & $50 \mathrm{~W}$ & $\boldsymbol{\sigma}$ & $L=39$ \\
\hline 2366 & 10.60 & .41 & .53 & .55 & 1.04 & 12.40 & .48 & .35 & .55 & .88 & S0T & $\boldsymbol{\theta}$ & 2366 \\
\hline L567 & 11.40 & 1.21 & 1.57 & .58 & 1.04 & 16.00 & 3.12 & 2.29 & .00 & .00 & $50 \%$ & $\boldsymbol{\theta}$ & L567 \\
\hline 2616 & 15.20 & 1.01 & 1.31 & .45 & .85 & 14.00 & 1.12 & .82 & .00 & .00 & $50 \%$ & $\boldsymbol{\theta}$ & L616 \\
\hline
\end{tabular}

GR. MBAN - 10.19 TAX NUMBER ORAND MBAN - 12.88 DAX NOMBER SD MANS . .77 WAX NOMBBR AFERAOB SDR - . 53 VAX NUMBER

TOTAL NOMBER OP LABORATSRIBE RBPGRTING • 20

Best values: $35310.1+1.2$ wax number

$\mathrm{J} 5512.8 \mp 2.5$ wax number

IURPACB PICE 8TRBNOTB, VAI NOMBB

TAPPI STANDARD TA89 OS-75, SURFACB BTRBNGTE OP PAPBR (VAX PICE TBST)

\begin{tabular}{|c|c|c|c|c|c|c|c|c|c|c|c|c|c|c|}
\hline $\mathrm{LAB}$ & & was & & COORDI & NATE 8 & $\triangle \nabla 0$ & & & & & & & & \\
\hline CEDB & $\mathbf{F}$ & $\sqrt{53}$ & $\sqrt{55}$ & MAJOR & MINOR & $R_{\bullet} \mathbf{8 D R}$ & $\mathbf{V A R}$ & PFOPB & RTY $=-$ & TB8T INBT & RONB? & $A T=--\cos$ & ADITIC & INS \\
\hline L243 & $\bullet$ & 8.40 & 9.60 & -3.71 & -42 & 1.44 & $50=$ & SURP $\triangle C E$ & P ICE & ETRENOTE. & $\nabla x$ & ( IAPPI & 1459 & $6975)$ \\
\hline 2230 & 6 & 9.20 & 12.40 & -082 & .74 & .87 & sov & SORPACB & PICI & SIRBNOTH, & $\nabla \Delta x$ & ( IAPPI & T459 & $6575)$ \\
\hline L1 22 & $\sigma$ & 9.60 & $13 \cdot 40$ & .26 & .74 & .96 & $50 \pi$ & SURP $\triangle C B$ & PICE & SIRENGTH, & NAx & ( IAPPI & 1459 & (6s75) \\
\hline L158 & $\boldsymbol{E}$ & 9.60 & 12.60 & .048 & .44 & 1.29 & $50=$ & BURPACB & PICK & BTRENGTH, & $\nabla \mathbf{A X}$ & ( TAPP I & 1459 & 6875) \\
\hline 2228 & 6 & 9.60 & 10.40 & -2.52 & .039 & .96 & 507 & GUEPACE & PICE & STRBNGTE, & $\mathbf{v \Delta x}$ & ( IAPP I & 1459 & (6975) \\
\hline 1339 & 0 & 9.80 & 13.20 & .15 & .48 & 1.31 & 500 & BURPACB & PICE & STRENGTH, & $m \Delta x$ & ( I $\triangle P P I$ & 1459 & 69751 \\
\hline L236 & 6 & 9.80 & $13 \cdot 40$ & .33 & .56 & .87 & 500 & SURP $\triangle C B$ & PICE & STREWOTH. & $\Delta x$ & ( IAPP I & 1459 & 69751 \\
\hline 2225 & $\boldsymbol{0}$ & 9.80 & 13.00 & .04 & .48 & 1.00 & $80=$ & 8URPACB & P ICE & STRENOTH. & $\mathbf{W A x}$ & ( I $\triangle P P P I$ & $T 459$ & $0975)$ \\
\hline 2274 & 0 & 10.00 & 13.20 & .22 & .30 & - 36 & 500 & SURP $\triangle C B$ & PICE & ATRBNOTH. & $\operatorname{Ax}$ & ( TAPP I & $T 459$ & 6875 \\
\hline 2183 & 0 & 10.00 & 13.20 & .22 & .30 & .68 & son & BURP $\triangle C B$ & PICE & STRBHOTH, & WII & (IAPPI & $T 459$ & $6875)$ \\
\hline 162 & 0 & 0.00 & $13 \cdot 40$ & .41 & .37 & 1.39 & 50w & SORPACB & PICE & STRENOTA, & $\Delta x$ & ( IAPPI & 1459 & $0875)$ \\
\hline L213 & $\theta$ & 10.20 & 13.80 & .86 & .34 & 1.85 & 500 & BURFACB & PICE & ETRBNOTB. & $\Delta x$ & ( TAPPI & T459 & $6875)$ \\
\hline 1366 & 0 & 10.60 & 12.40 & -.29 & -.86 & .96 & $50 \pi$ & SURF $\triangle C B$ & PICE & STRBNOTB. & WI & ( IAPPI & T459 & $6875)$ \\
\hline L1734 & 0 & 10.00 & $12 \cdot 20$ & 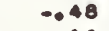 & -.64 & .88 & 500 & SORFACB & PICE & SIRBNOTE, & $\Delta \mathrm{I}$ & ( IAPP I & $T 459$ & 68751 \\
\hline 2195 & 0 & 10.80 & 12.80 & .16 & .60 & .79 & sow & $8 O R P \triangle C B$ & PICE & ATRBNOTE, & max & ( TAPP I & I459 & $0875)$ \\
\hline L105 & $\sigma$ & 11.00 & 11.40 & 1.07 & $-1 \cdot 31$ & $1 \cdot 12$ & sov & SURPACB & P ICE & STRBNOTE, & $\nabla \Delta x$ & ( IAPP I & 1459 & (6575) \\
\hline L1 82W & 0 & 18.00 & 12.80 & .23 & .078 & .36 & sov & SORPACB & PICE & 8TRBNGTH, & DAX & ( IAPP I & 1459 & 6875) \\
\hline 2616 & $\sigma$ & 11.20 & 14.00 & 1.42 & -081 & .43 & sow & SURFACB & PICE & STREHOTB。 & $w A x$ & ( TAPP I & T459 & 68751 \\
\hline 2285 & $\sigma$ & 11.20 & 14.40 & 1.79 & -.36 & 1.96 & 500 & SURPACB & PICE & ETPEHGTE, & $\nabla \Delta x$ & ( IAPPI & 1459 & (6875) \\
\hline LS67 & 0 & 11.40 & 16.00 & 3.35 & .05 & $\bullet 52$ & $50 \pi$ & BURP $\triangle C B$ & PICE & SIRBNGTE, & $\nabla \Delta x$ & ( TAPP I & T459 & 69751 \\
\hline GME & & $\begin{array}{c}10.19 \\
95 \%\end{array}$ & $\begin{array}{l}12 \cdot 88 \\
L I P 8 B\end{array}$ & . 98 & .61 & I I & & o & & & & & & \\
\hline
\end{tabular}


SURFACE PICK STRENGTH, WAX

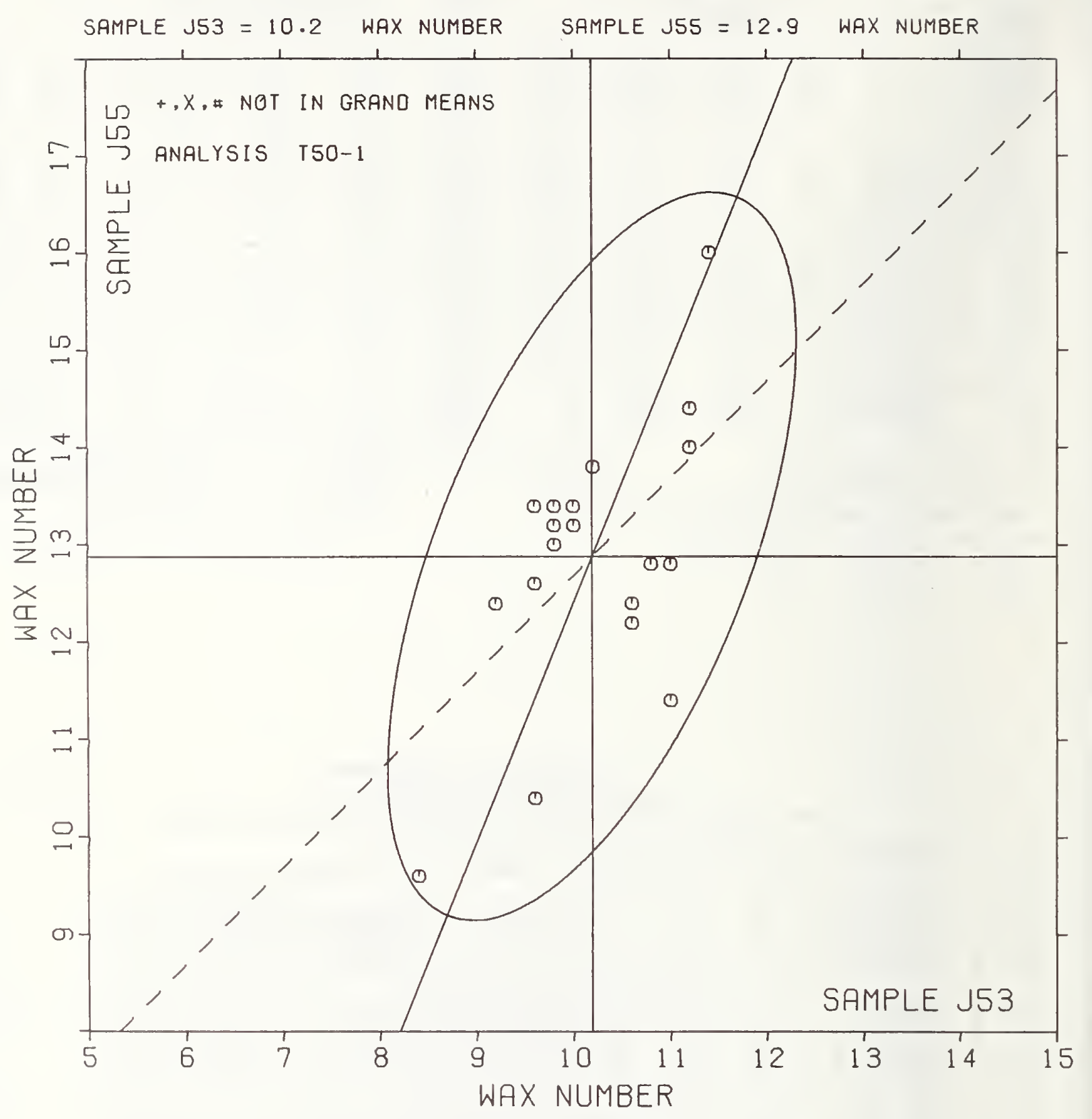


TAPPI STANDARD T809 69.71

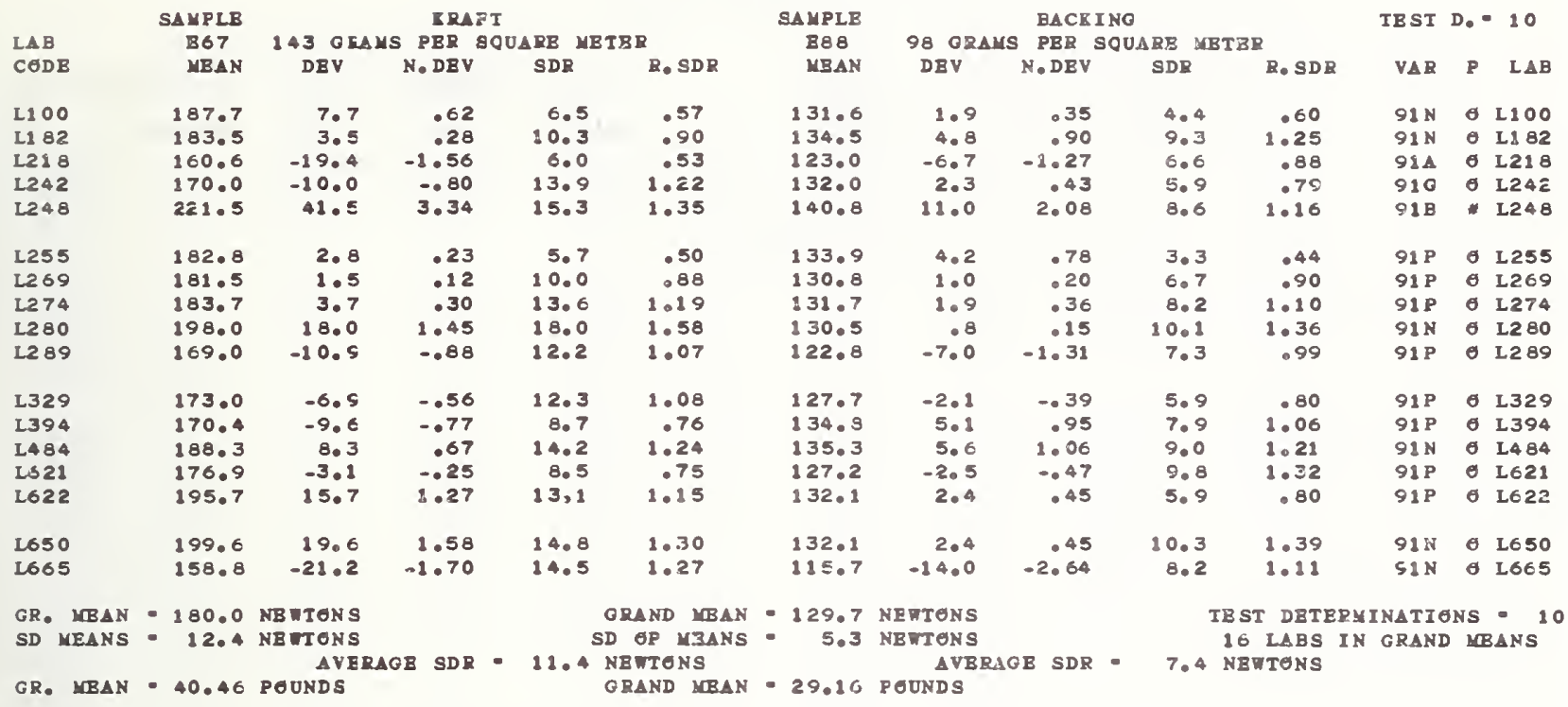

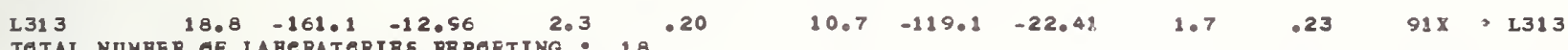

Best values: E67 $180+20$ newtons

E88 $130 \pm 7$ newtons

The following laboratories were omitted from the

grand means because of extreme test results: 248.

RBPORT NG. 575 IAPPI COLLABGRATIVE RBFERENCB PREGRAM
ANALYSIS I91-1 IABLB 2

CENCERA (CERROGATING MBDIUM TBST-CMT) IAPPI STANDARD T809 6S-71

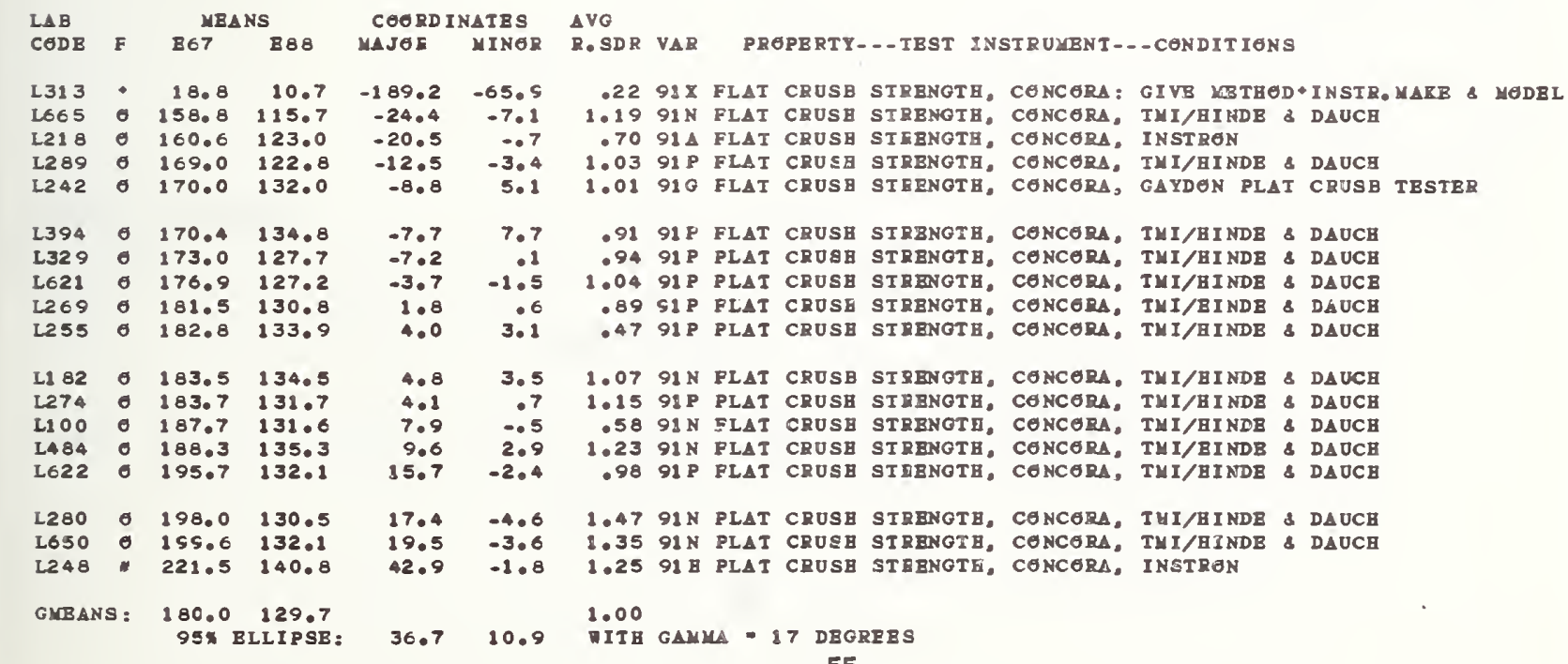




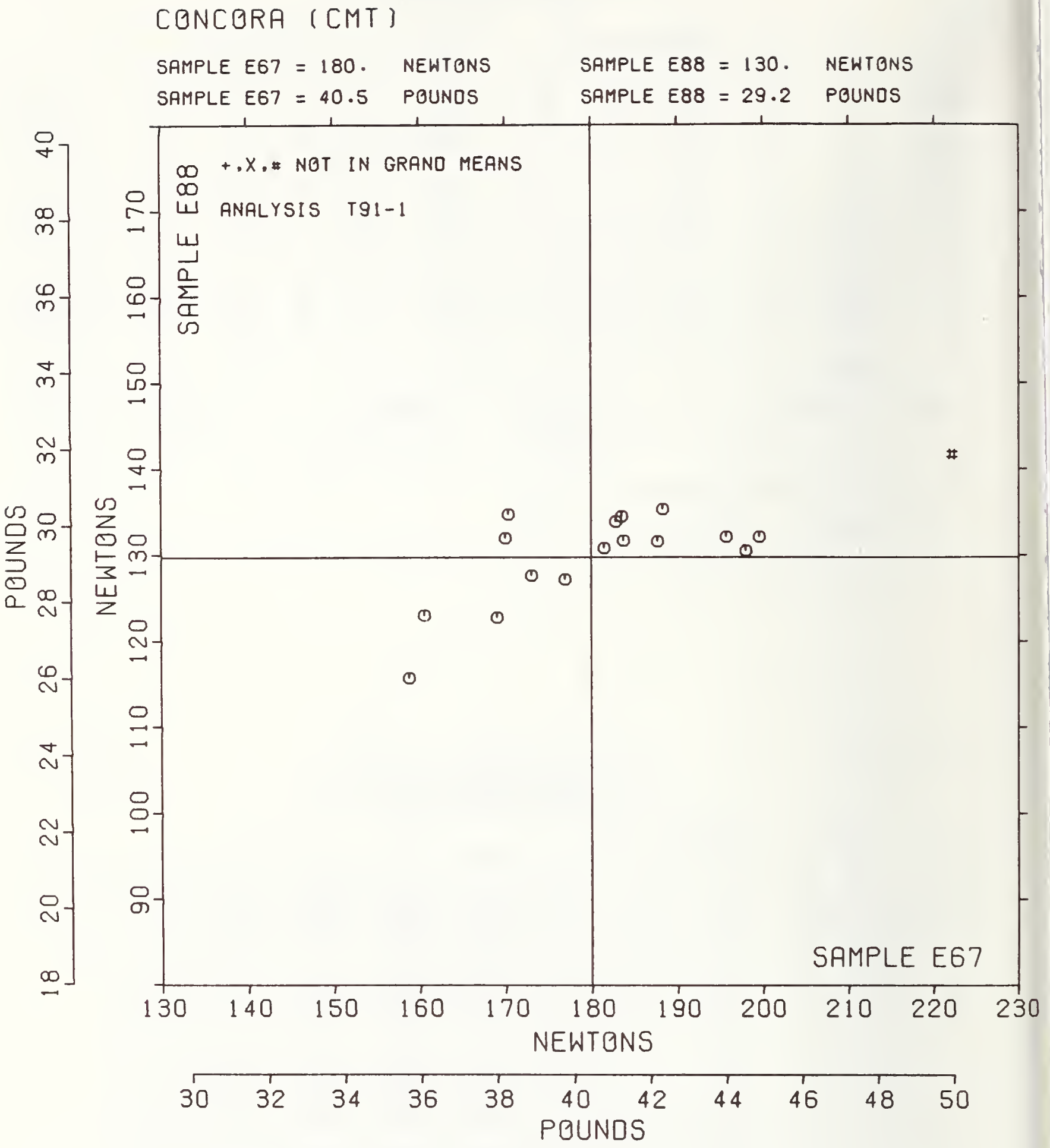


TAPPI STANDARD TEIB O8-76

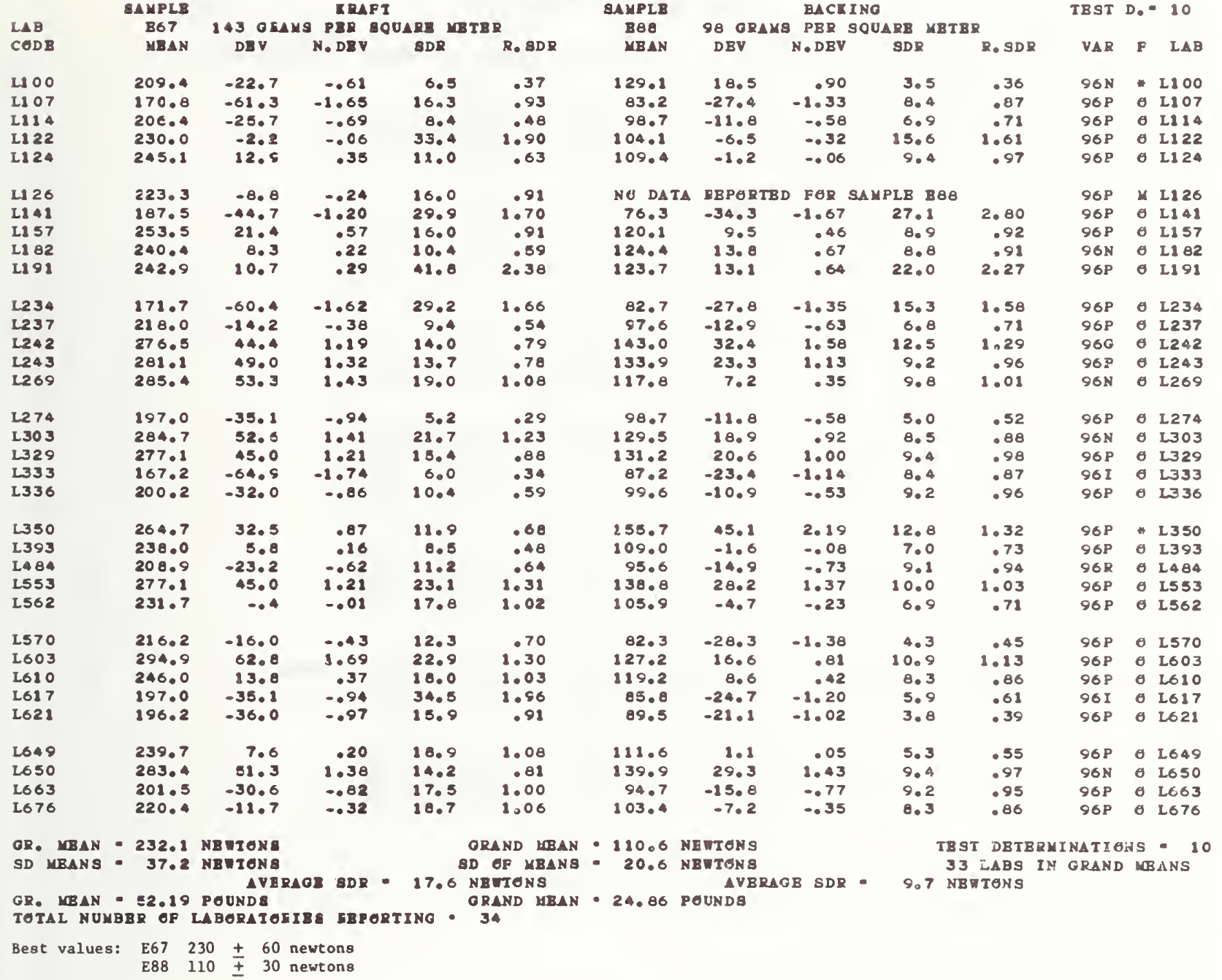


RINO CRUSA (COMPRABSIEN RBSIBIANCB OP PAPERBOARD)

TAPPI STANDARD T818 68.76

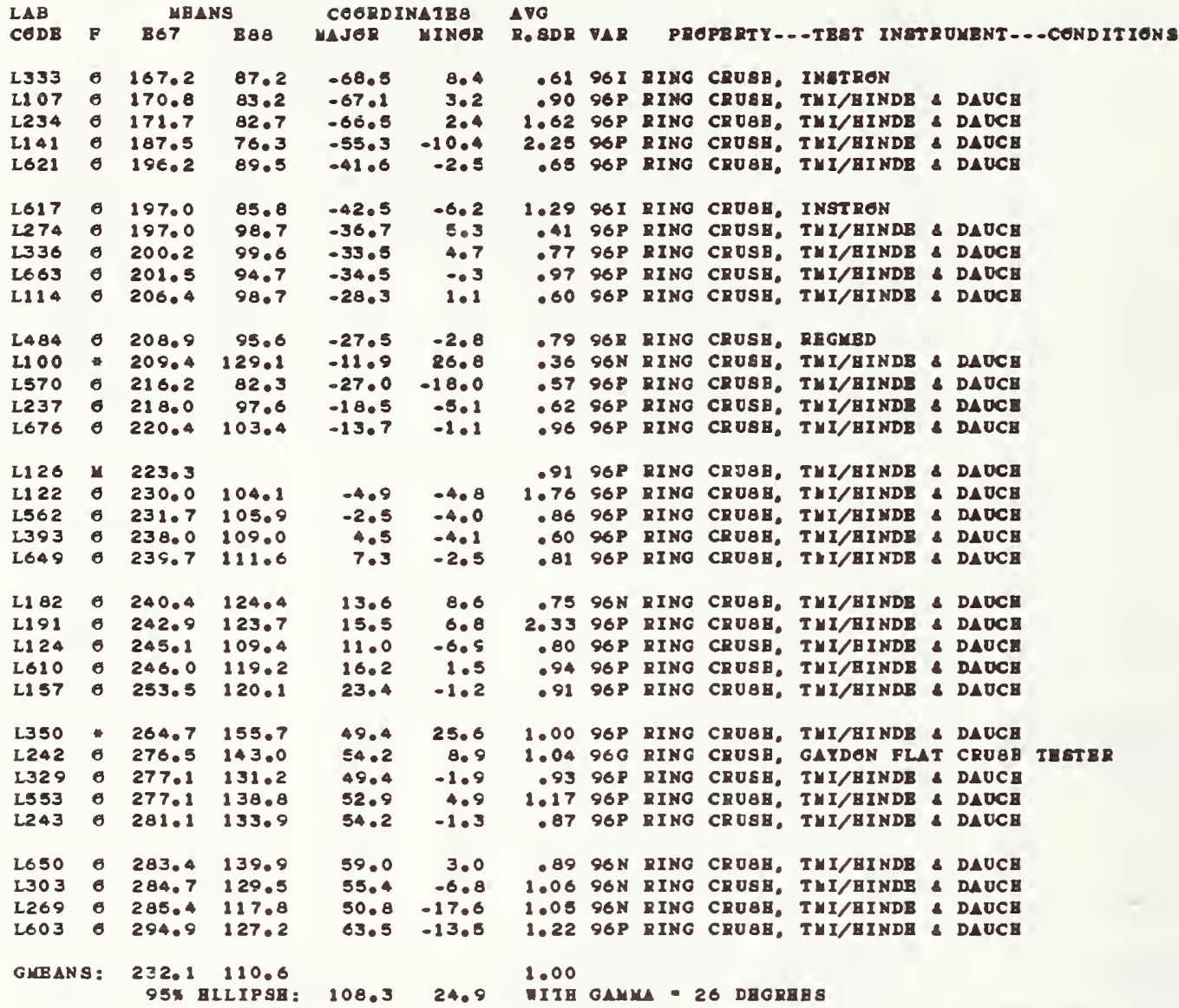


RING CRUSH

SAMPLE E67 $=232$. NEWTONS SAMPLE E88 $=111$. NEWTONS

SAMPLE E67 $=52.2$ POUNDS SAMPLE E88 $=24.9$ POUNDS

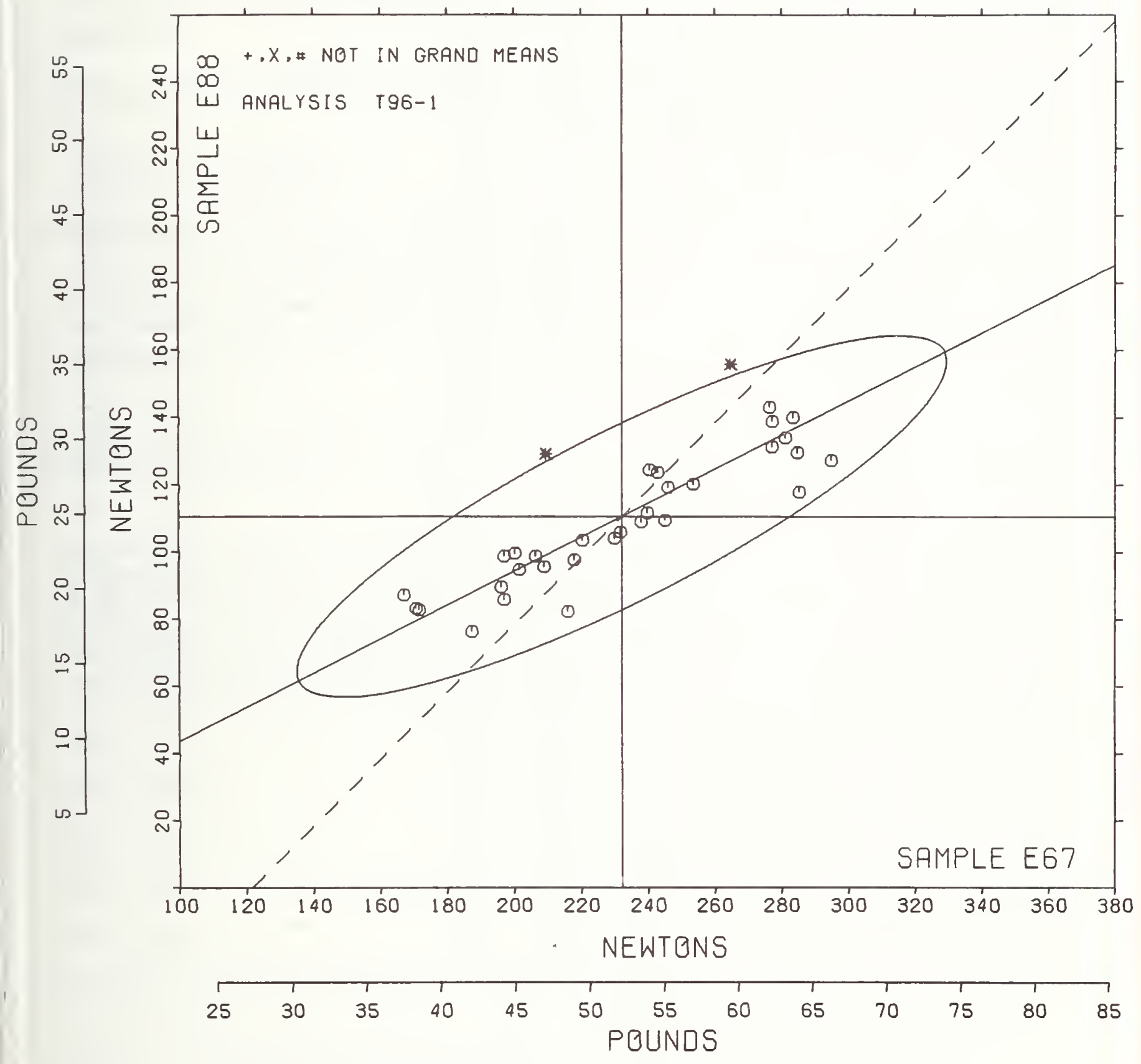




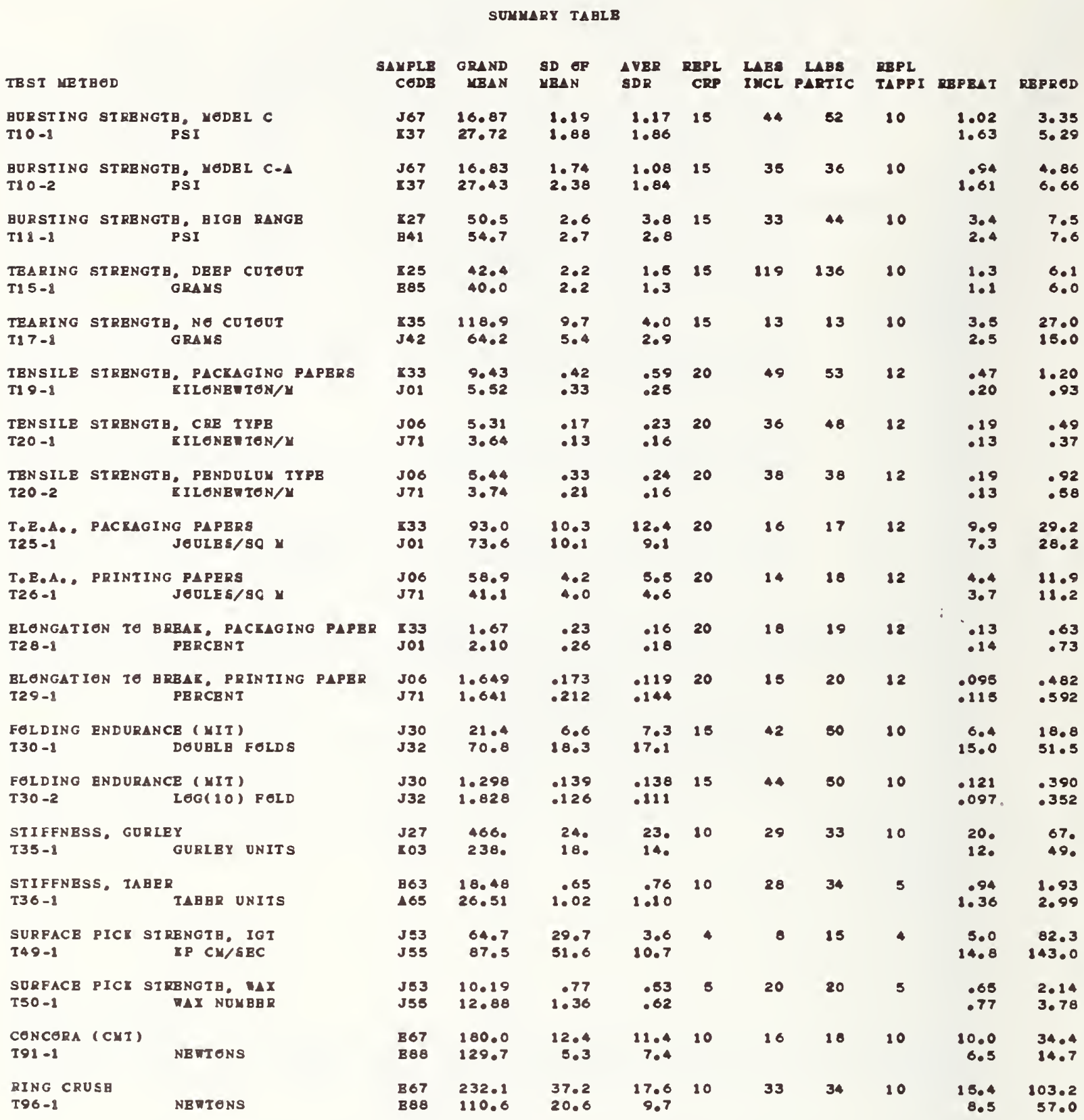


NBS. IIAA (REV. 9.78 )

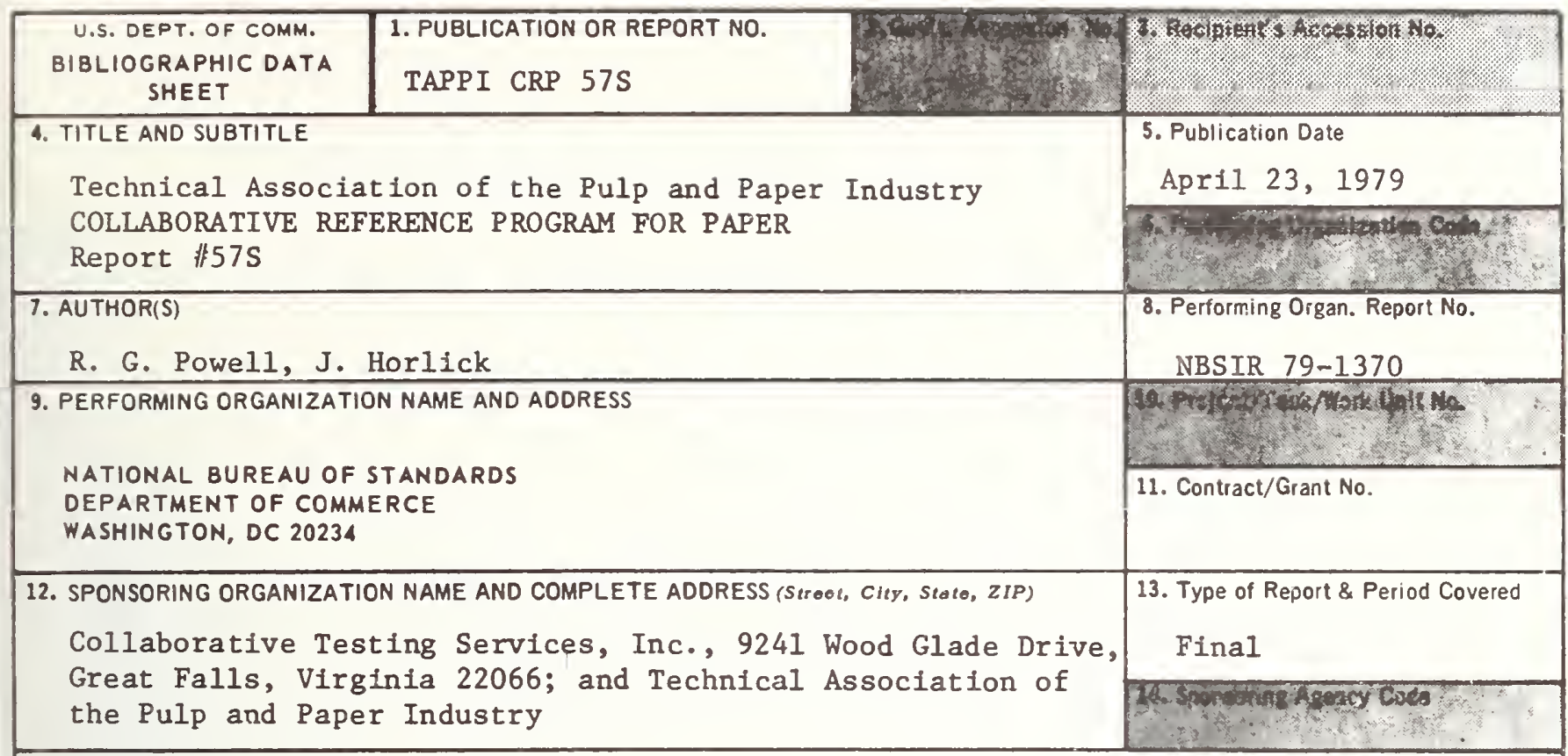

15. SUPPLEMENTARY NOTES

Document describes a computer program; SF-185, FIPS Software Summary, is attached.

16. ABSTRACT (A 200-word or lose factuai aummary of most significant information. If document includes a significant bibliography or litosature survey, mention it here.)

Collaborative Reference Programs provide participating laboratories with the means for checking periodically the level and uniformity of their testing in comparison with that of other participating laboratories. An important by-product of the programs is the provision of realistic pictures of the state of the testing art. This is one of the periodic reports showing averages for each participant, within and between laboratory variability, and other information for participants and standards comittees.

17. KEY WORDS (aix to twelvo onertes; alphabotical order; capilalise only the firot letter of the firat koy word unleas a propor namo; soparatod by amicotono)

Collaborative reference program; Laboratory evaluation; Paper; Precision; Reference samples; Testing calibration

\begin{tabular}{|c|c|c|}
\hline 18. AVAILABILITY $\square$ Unllmited & $\begin{array}{l}\text { 19. SECURITY CLASS } \\
\text { (THIS REPORT) }\end{array}$ & $\begin{array}{l}\text { 21. NO. OF } \\
\text { PRINTED PAGES }\end{array}$ \\
\hline X For Official Distribution. Do llot Release to NTIS & UNCLASSIFIED & 67 \\
\hline $\begin{array}{l}\text { Order From Sup. of Doc., U.S. Government Printing Office, Wasinington, DC } \\
20402 \text {, SD Stock No. SNO03-003- }\end{array}$ & $\begin{array}{l}\text { 20. SECURITY CLASS } \\
\text { (THIS PAGE) }\end{array}$ & 22. Price \\
\hline $\begin{array}{l}\square \text { Order From National Technical Information Service (NTIS), Springfield, } \\
\text { VA. } 22161\end{array}$ & UNCLASSIFIED & \\
\hline
\end{tabular}


This diagram is composed of two fullsize overlaid tracings. One tracing was made from the Thwing-Elmendorf tear tester with NO CUTOUT (old style). The other tracing was made from the Thwing-Elmendorf tear tester with DEEP CUTOUT. The cross hatched area represents the metal removed from the swinging sector when the deep cutout (new) style was created.

DEEP CUTOUT instrument is $5 / 8$ inch across NO CUTOUT instrument is $11 / 4$ inch across

Note shape of pendulum sector with respect to an imaginary line drawn across the top of the specimen clamp 
